\title{
Domain redistribution in lipid bilayers in the presence of the antimicrobial lipopeptide fengycin
}

By

\section{Elisabeth Mantil}

A thesis submitted to the Faculty of Graduate and Postdoctoral Affairs in partial fulfillment of the requirements for the degree of

Doctor of Philosophy

in

Chemistry

(Concentration in Food Science and Nutrition)

Carleton University

Ottawa, Canada

(C) 2018, Elisabeth Mantil 


\section{Abstract}

The general objective of this research was to gain insight into the mechanisms of action of the antimicrobial cyclic lipopeptide fengycin on model membranes. Total internal reflection fluorescence (TIRF) microscopy was used to visualize and analyse lipid phase separation within supported lipid bilayers (SLBs) of various compositions. The initial research investigated the influence of protocol parameters on fluorescent probe distribution and lipid domain appearance within SLBs. The influence of sonication and extrusion during liposomal preparations, choice of solid support containment, and ratio of unsaturated and saturated phospholipids (DOPC:DPPC) in SLBs were assessed. Liposomal solutions passed through an extruder after sonication saw a decrease in TR-DHPE aggregates. Bilayers composed of a 3:1 DOPC:DPPC with $0.8 \%$ mol TRDHPE produced bilayers with even fluorescence, distinct phase separations, and limited photobleaching. Using this optimized protocol, ergosterol was incorporated into the bilayers and domain redistribution was evaluated after the addition of fengycin. SLBs containing higher concentrations of ergosterol were more tolerant to changes induced by fengycin. Following the analysis of these ternary SLBs, extracted lipids from molds with different fengycin sensitivity were use in the preparation of SLBs. Bilayers composed of Alternaria solani lipids were most tolerant to fengycin. A. solani also contained the highest level of ergosterol compared to Fusarium sambucinum and Pythium sulcatum. The redistribution of lipids phases it these membranes could also be attributed to the readsorption of phospholipid-fengycin micelles produced during bilayer solubilization. Overall, the results of these works confirm that ergosterol plays a key role in the antimicrobial activity of fengycin. An increase in ergosterol content strongly correlates with an increase in ordered phases, potentially promoting membrane insolubility as seen in surfactant resistant membranes. 


\section{Acknowledgements}

I would like to express my deepest gratitude to my thesis supervisor Dr. Tyler Avis for continuous guidance, support, and advice while completing my thesis. His patience throughout learning new techniques, and while I developed a love-hate relationship with the TIRF microscope, was greatly appreciated. The encouragement received from fellow lab and office mates, was vital on my path to completing this thesis.

I would also like to thank my family and friends who were a constant source of support and encouragement. While I experienced some ups and downs while completing my graduate studies, they were always there. 


\section{Table of Contents}

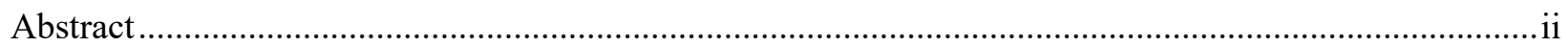

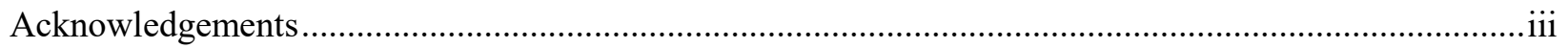

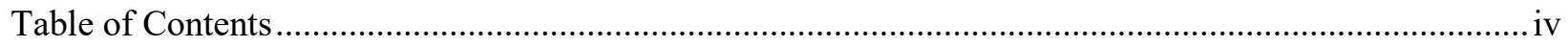

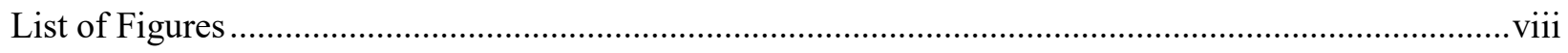

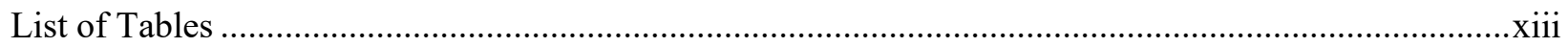

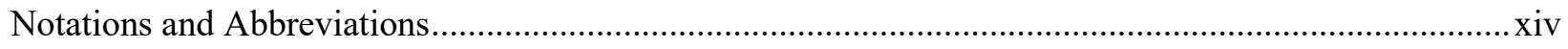

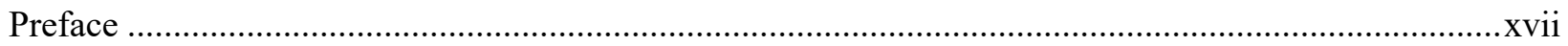

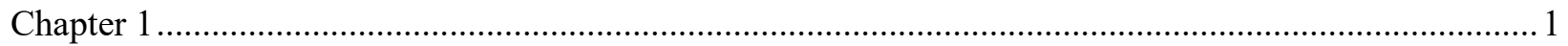

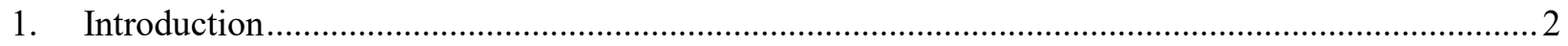

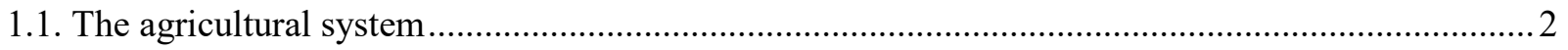

1.1.1. Pathogenic fungi and their impact on crop yield and food spoilage ......................................2

1.1.2. Current control methods and potential drawbacks................................................................. 4

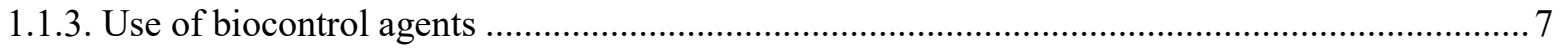

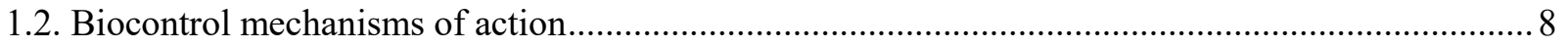

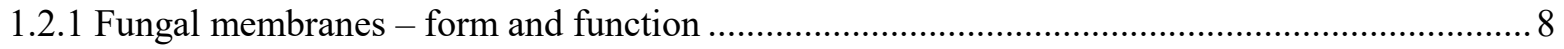

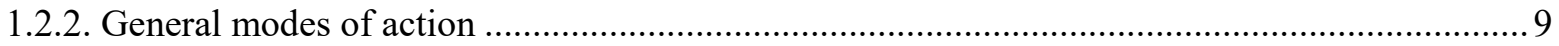

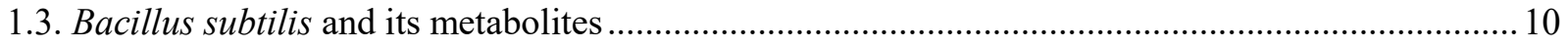

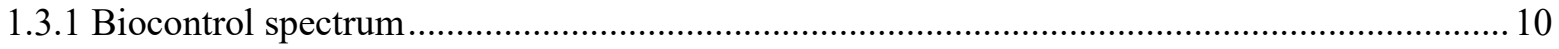

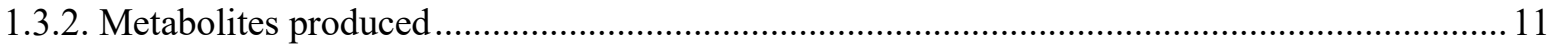

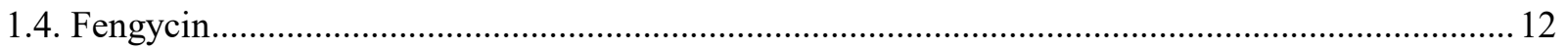

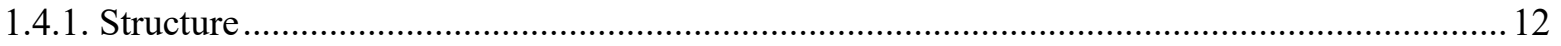

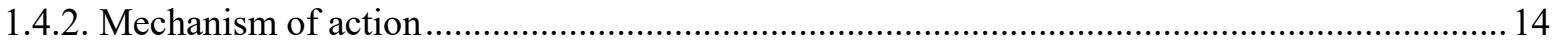

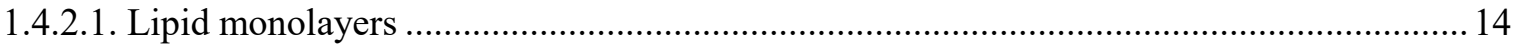

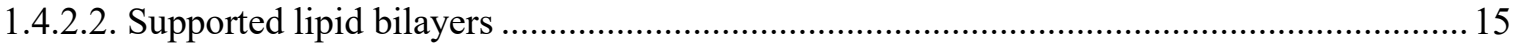

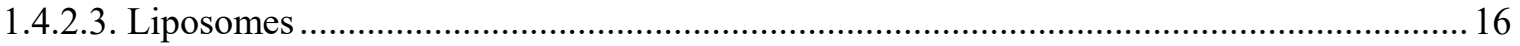

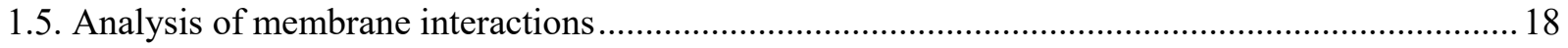

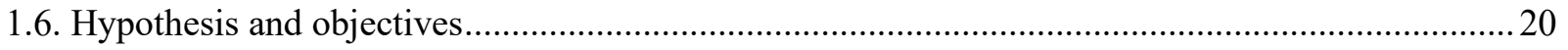

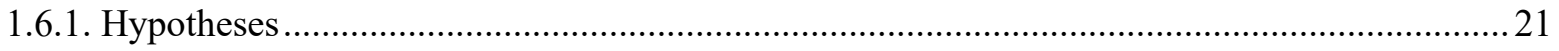

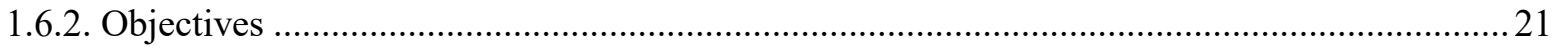

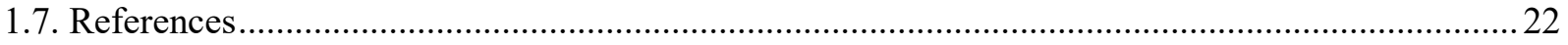




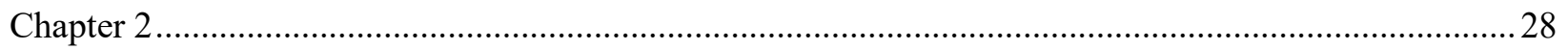

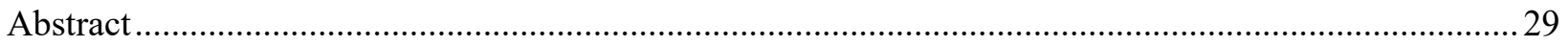

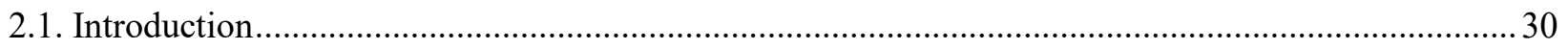

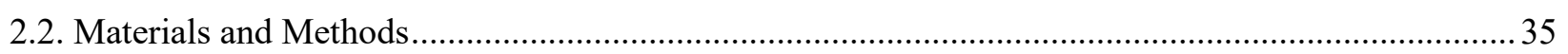

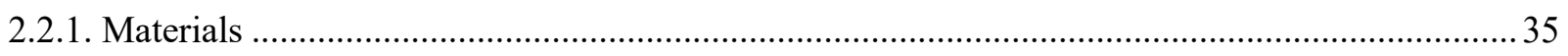

2.2.2. Small Unilamellar Vesicle (SUV) Preparation ..................................................................... 36

2.2.2.1. General Vesicle Preparation Protocol ................................................................................ 36

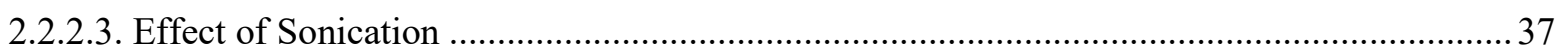

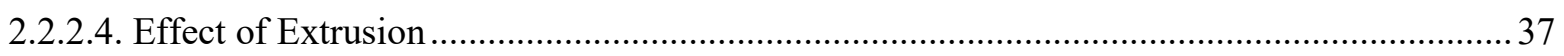

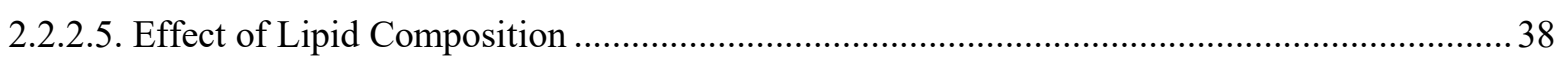

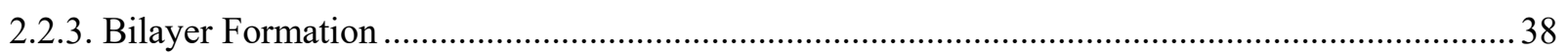

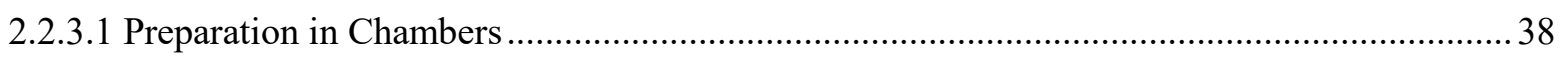

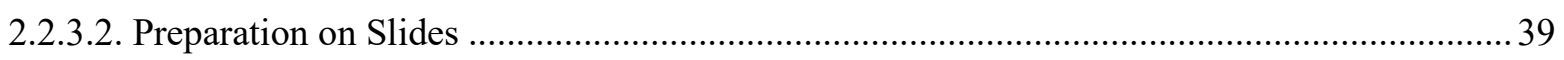

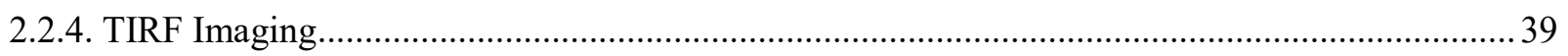

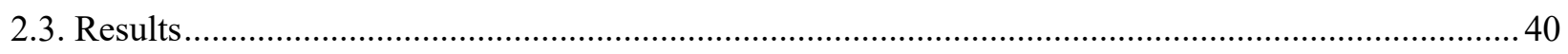

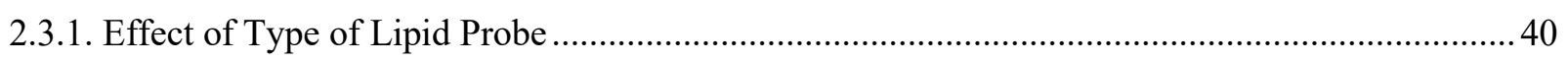

2.3.2. Effects of Lipid Probe (TR-DHPE) Concentration and Sonication ............................................ 41

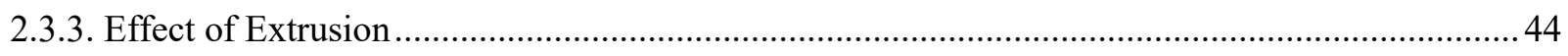

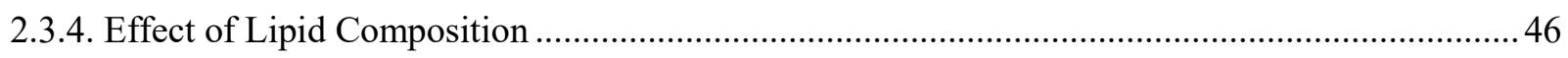

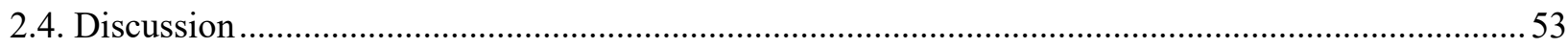

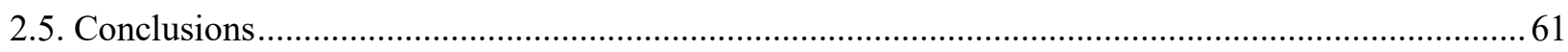

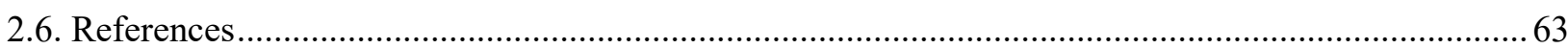

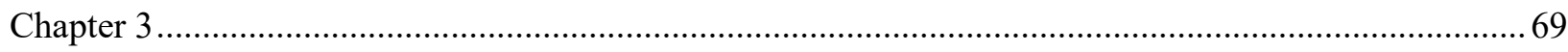

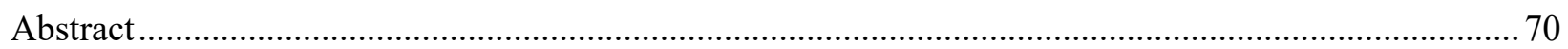

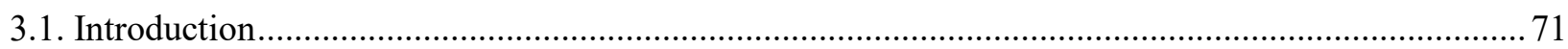

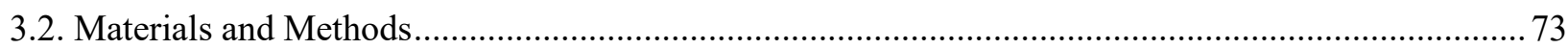

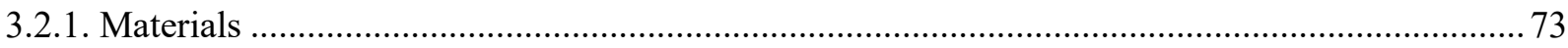

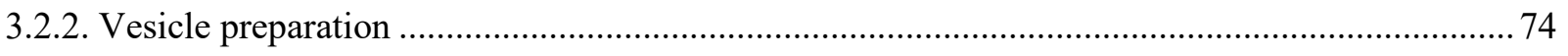

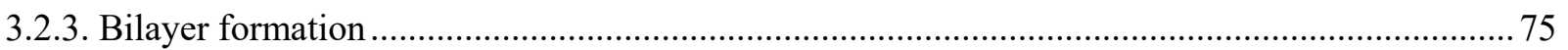

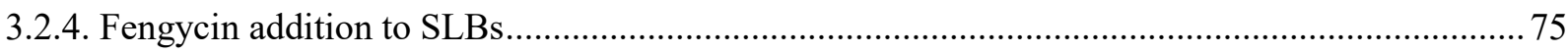

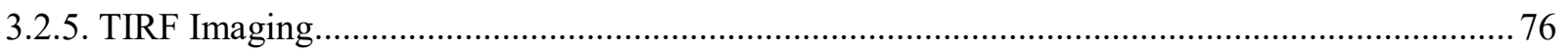

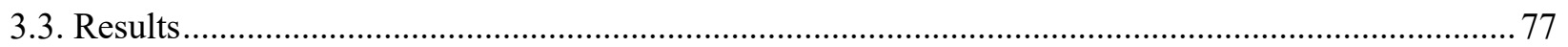

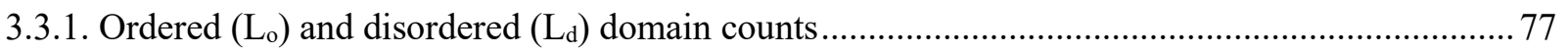

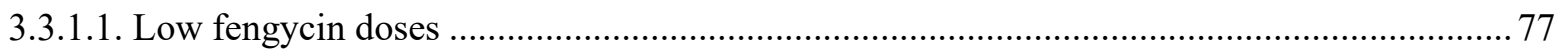




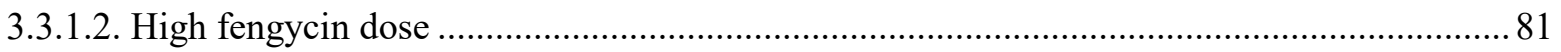

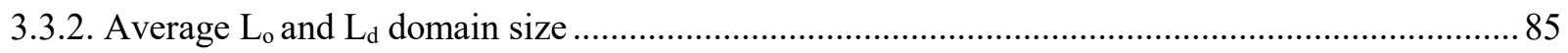

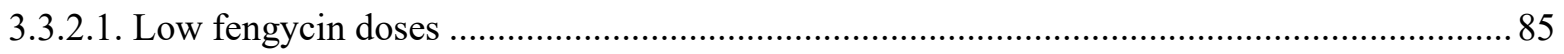

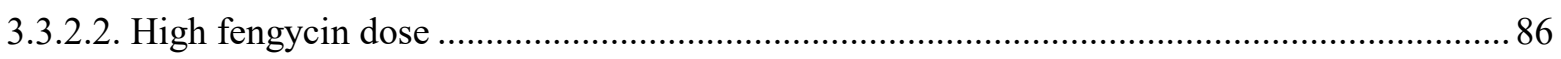

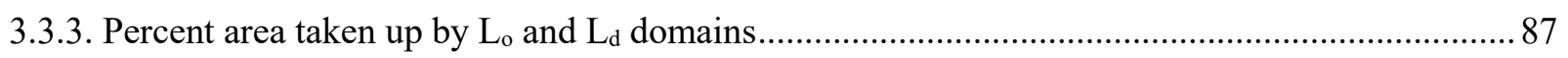

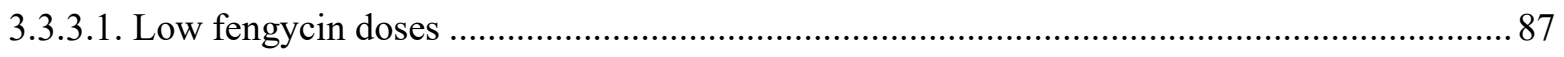

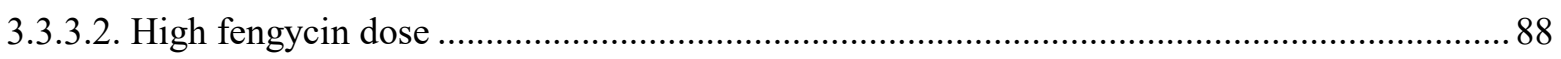

3.3.4. General effect of fengycin on membrane characteristics .................................................... 89

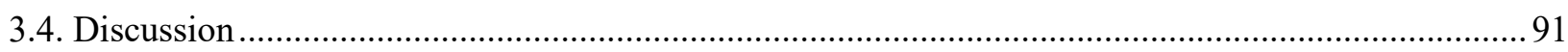

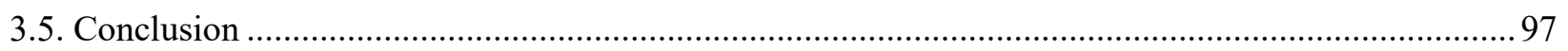

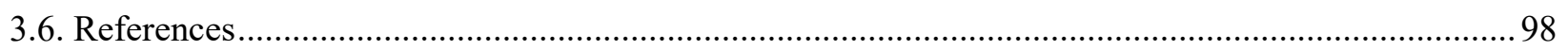

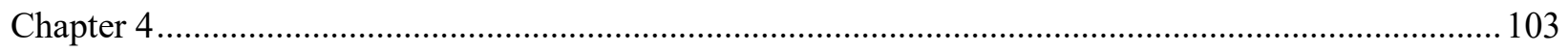

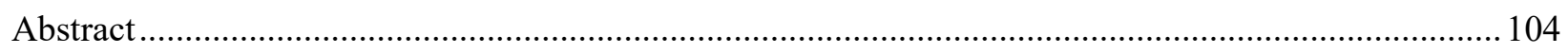

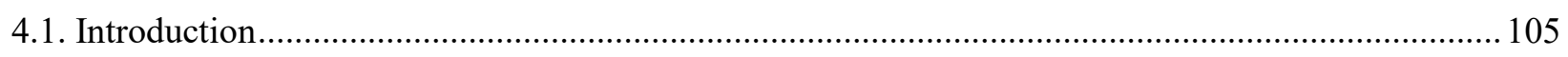

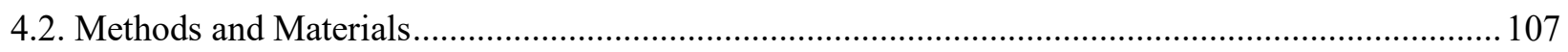

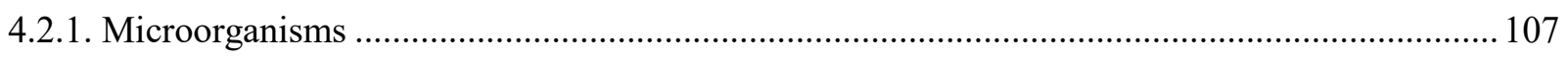

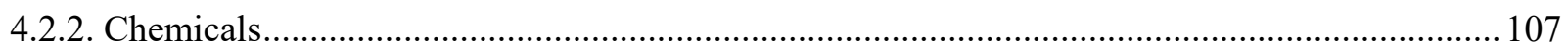

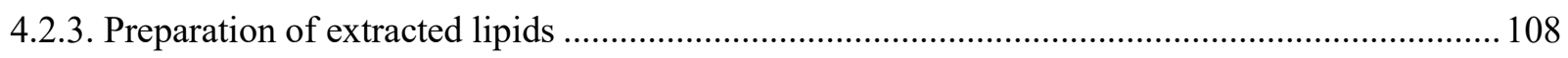

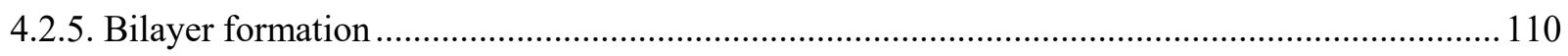

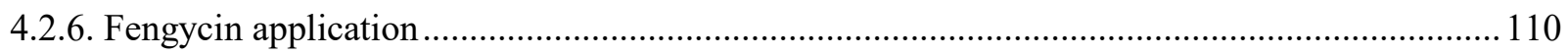

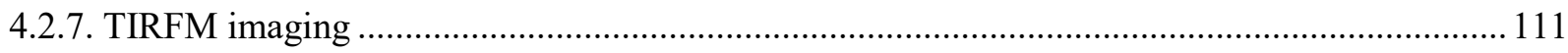

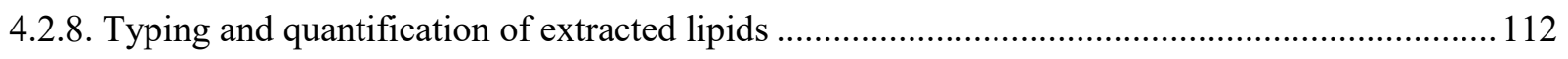

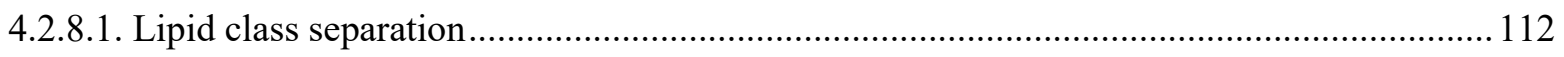

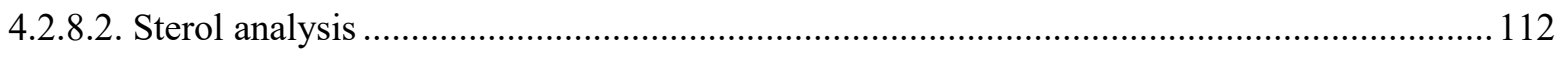

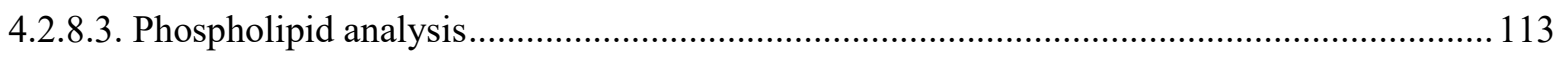

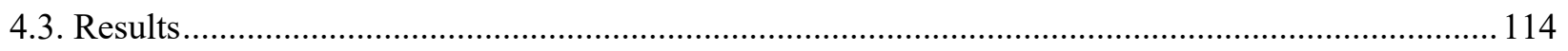

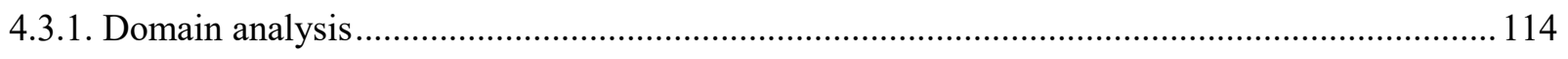

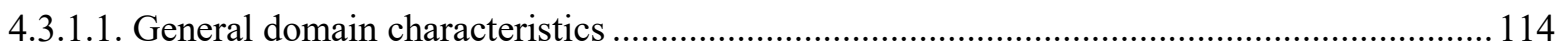

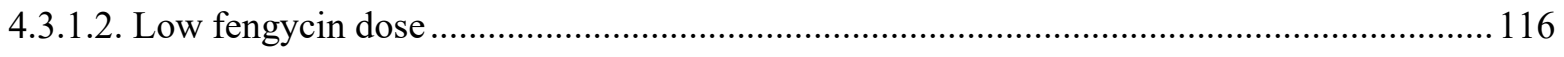

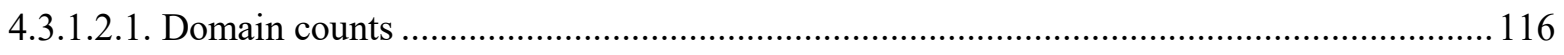

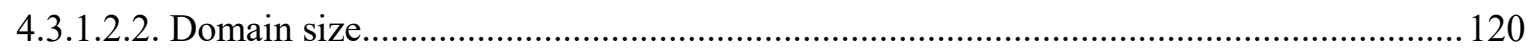

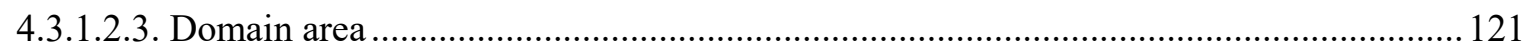

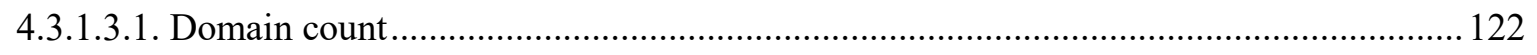

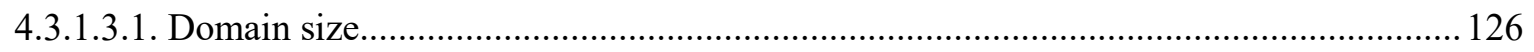




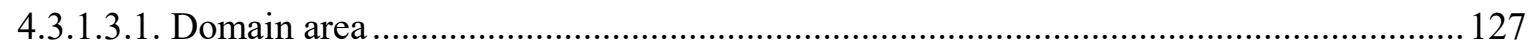

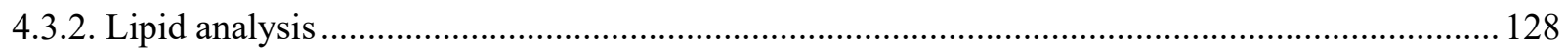

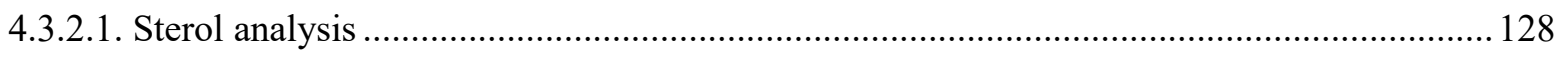

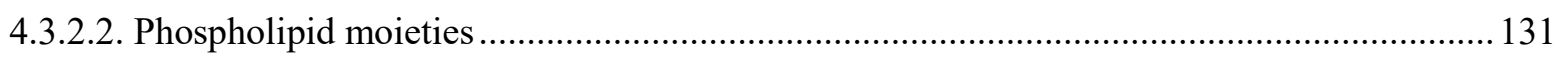

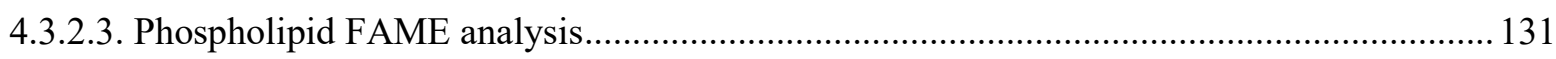

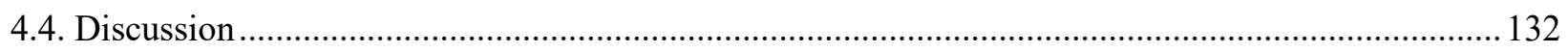

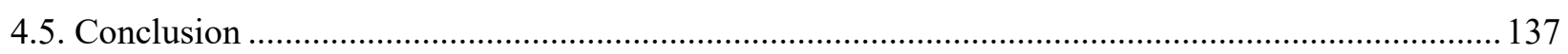

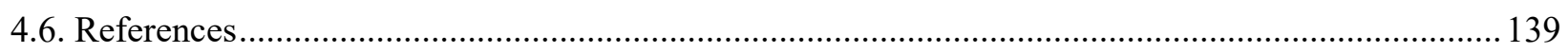

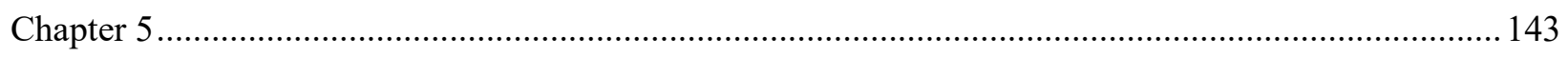

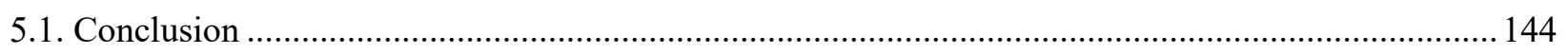

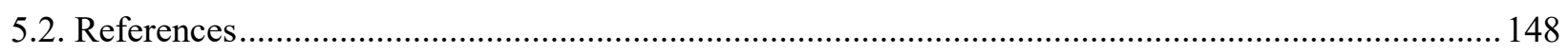

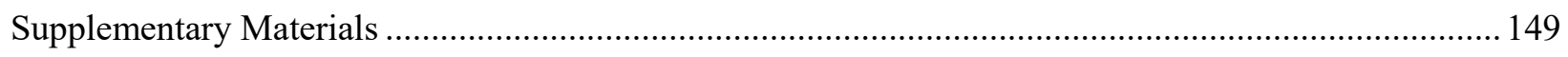




\section{List of Figures}

\section{Chapter 1}

Figure 1.1. Population of the world: estimates, 1950-2015, and medium-variant projection with 95 per cent prediction intervals, 2015-2100 [1]. 2

Figure 1.2. General structure of the fengycin A molecule. Table depicts the variances in amino acid residues of the cyclic peptide. 13

\section{Chapter 2}

Figure 2.1. Experimental setups use for Total Internal Reflection Fluorescence Microscopy. A. Chamber apparatus containing the lipid bilayer coated coverslip. B. Microscope slide setup containing the lipid bilayer. Red = phospholipid bilayer; blue = aqueous layer covering the bilayer.

Figure 2.2. Effect of fluorescent probe concentration and sonication time on supported lipid bilayer visualization using the chamber setup. Bilayers were prepared using 1:1 DOPC:DPPC lipid solutions containing 0.1-0.8\% mol TR-DHPE, as indicated. Lipid solutions were prepared using one (a, c, e, g, i, k, m, o) or two hours (b, d, f, h, j, l, n, p)of sonication. Scale bars $=1 \mu \mathrm{m} . \ldots . . .42$ Figure 2.3. Effect of fluorescent probe concentration and sonication time on supported lipid bilayer visualization using the slide setup. Bilayers were prepared using 1:1 DOPC:DPPC lipid solutions containing $0.1-0.8 \%$ mol TR-DHPE, as indicated. Lipid solutions were prepared using one $(a, c, e, g, h, i, k, m)$ or two hours $(b, d, f, j, 1, n)$. Scale bars $=1 \mu \mathrm{m}$.

Figure 2.4. Effect of extrusion on supported lipid bilayer visualization. Bilayers were prepared using 1:1 DOPC:DPPC lipid solutions containing 0.8\% mol TR-DHPE. Bilayers were visualized using the chamber $(a, b)$ and slide $(b, c)$ setup. Lipid solutions were prepared using sonication (a, c) or sonication with an additional extrusion process (b, d). Scale bars $=30 \mu \mathrm{m}$. Green images (a, b) visualized with a $473 \mathrm{~nm}$ laser and a multicube; red images (c, d) visualized with a $568 \mathrm{~nm}$ laser and a $561 \mathrm{~nm}$ cube. 45

Figure 2.5. Effect of pure DOPC lipid composition on supported lipid bilayer visualization. Bilayers were prepared using $0.8 \%$ mol TR-DHPE. Bilayers were visualized using the chamber 
$(a, b)$ and slide $(b, c)$ setup. Lipid solutions were prepared using sonication $(a, c)$ or sonication with an additional extrusion process $(\mathrm{b}, \mathrm{d})$. Scale bars $=30 \mu \mathrm{m}$. Green images $(\mathrm{a}, \mathrm{b})$ visualized with a $473 \mathrm{~nm}$ laser and a multicube; red images (c, d) visualized with a $568 \mathrm{~nm}$ laser and a $561 \mathrm{~nm}$ cube.

Figure 2.6. Effect of 3:1 DOPC:DPPC lipid composition on supported lipid bilayer visualization. Bilayers were prepared using 0.8\% mol TR-DHPE. Bilayers were visualized using the chamber $(a, b)$ and slide $(b, c)$ setup. Lipid solutions were prepared using sonication $(a, c)$ or sonication with an additional extrusion process $(\mathrm{b}, \mathrm{d})$. Scale bars $=30 \mu \mathrm{m}$. Images visualized with a $568 \mathrm{~nm}$ laser and a $561 \mathrm{~nm}$ cube.

Figure 2.7. Effect of 1:3 DOPC:DPPC lipid composition on supported lipid bilayer visualization. Bilayers were prepared using 0.8\% mol TR-DHPE. Bilayers were visualized using the chamber $(\mathrm{a}, \mathrm{b})$ and slide $(\mathrm{b}, \mathrm{c})$ setup. Lipid solutions were prepared using sonication $(\mathrm{a}, \mathrm{c})$ or sonication with an additional extrusion process $(b, d)$. Scale bars $=30 \mu \mathrm{m}$. Images visualized with a $568 \mathrm{~nm}$ laser and a $561 \mathrm{~nm}$ cube. 51

Figure 2.8. Effect of pure DPPC lipid composition on supported lipid bilayer visualization. Bilayers were prepared using 0.8\% mol TR-DHPE. Bilayers were visualized using the chamber $(a, b)$ and slide $(b, c)$ setup. Lipid solutions were prepared using sonication $(a, c)$ or sonication with an additional extrusion process $(\mathrm{b}, \mathrm{d})$. Scale bars $=30 \mu \mathrm{m}$. Green images (a, d) visualized with a $473 \mathrm{~nm}$ laser and a multicube; red images (b, c) visualized with a $568 \mathrm{~nm}$ laser and a $561 \mathrm{~nm}$ cube.

\section{Chapter 3}

Figure 3.1. Effect of low doses $(50 \mu \mathrm{g})$ of fengycin on liquid ordered $\left(\mathrm{L}_{\mathrm{o}}\right)$ domain characteristics and distribution of lipid bilayers. Bilayers contained 3:1 DOPC:DPPC with 0 (blue), 3 (orange), 6 (grey), and 12\% (yellow) ergosterol. Fengycin added at the 0 minute (dose 1) and the 40 minute marks (dose 2). A. Relative $\mathrm{L}_{\mathrm{o}}$ domain count; B. relative $\mathrm{L}_{\mathrm{o}}$ domain size; C. relative $\mathrm{L}_{\mathrm{o}}$ domain area.

Figure 3.2. Effect of low doses $(50 \mu \mathrm{g})$ of fengycin on liquid disordered $\left(\mathrm{L}_{\mathrm{d}}\right)$ domain characteristics and distribution of lipid bilayers. Bilayers contained 3:1 DOPC:DPPC with 0 (blue), 3 (orange), 6 (grey), and 12\% (yellow) ergosterol. Fengycin added at the 0 minute (dose 1 ) and the 
40 minute marks (dose 2). A. Relative $\mathrm{L}_{d}$ domain count; B. relative $\mathrm{L}_{\mathrm{d}}$ domain size; C. relative $\mathrm{L}_{\mathrm{d}}$ domain area

Figure 3.3. Effect of a high dose $(500 \mu \mathrm{g})$ of fengycin on liquid ordered $\left(\mathrm{L}_{\mathrm{o}}\right)$ domain characteristics and distribution of lipid bilayers. Bilayers contained 3:1 DOPC:DPPC with 0 (blue), 3 (orange), 6 (grey), and 12\% (yellow) ergosterol. Fengycin added at the 0 minute mark. A. Relative $\mathrm{L}_{\mathrm{o}}$ domain count; B. relative $\mathrm{L}_{\mathrm{o}}$ domain size; C. relative $\mathrm{L}_{\mathrm{o}}$ domain area. 82

Figure 3.4. Effect of a high dose $(500 \mu \mathrm{g})$ of fengycin on liquid disordered $\left(\mathrm{L}_{\mathrm{d}}\right)$ domain characteristics and distribution of lipid bilayers. Bilayers contained 3:1 DOPC:DPPC with 0 (blue), 3 (orange), 6 (grey), and 12\% (yellow) ergosterol. Fengycin added at the 0 minute mark. A. Relative $\mathrm{L}_{\mathrm{d}}$ domain count; B. relative $\mathrm{L}_{\mathrm{d}}$ domain size; C. relative $\mathrm{L}_{\mathrm{d}}$ domain area................... 84 Figure 3.5. Effect of $500 \mu \mathrm{g}$ of fengycin on lipid bilayers. Bilayers contain 3:1 DOPC:DPPC with 0 (A-D), 3 (E-H), 6 (I-L), and 12\% (M-P) ergosterol. Fengycin added immediately after the 0 minute mark. Bilayers were visualized using a $568 \mathrm{~nm}$ laser and $651 \mathrm{~nm}$ cube on a Total Internal Reflection Fluorescence (TIRF) microscope. Scale bar is $10 \mathrm{um}$. 90

\section{Chapter 4}

Figure 4.1. TIRFM images of SLBs before exposure to fengycin (A, D, G) after exposure to a low dose of fengycin (two $50 \mu \mathrm{g}$ applications) (B, E, H), and a high dose of fengycin (one $500 \mu \mathrm{g}$ fengycin application) (C, F, I). SLB were prepare from lipids extracts from A. solani (A-C), $F$. sambucinum (D-F), and P. sulcatum (G-I). Images visualized with a $568 \mathrm{~nm}$ laser and $561 \mathrm{~nm}$ cube. 115

Figure 4.2. TIRFM imaging of $F$. sambucinum SLBs during exposure to $500 \mu \mathrm{g}$ of fengycin, taken at (A) zero, (B) one, (C) five, (D) 20, and (E) 40 minutes. Images visualized using a $568 \mathrm{~nm}$ laser and $561 \mathrm{~nm}$ cube. 116

Figure 4.3. Effect of a low dose $(50 \mu \mathrm{g})$ of fengycin on liquid ordered (Lo) domain characteristics and distribution of lipid bilayers. Bilayers contained extracted lipids from Pythium sulcatum (blue), Alternaria solani (orange), and Fusarium sambucinum (grey). Fengycin added at 0 minute (dose 1) and the 40 minute (dose 2) marks. (A) $\mathrm{L}_{\mathrm{o}}$ domain count; (B) $\mathrm{L}_{\mathrm{o}}$ domain size; (C) $\mathrm{L}_{\mathrm{o}}$ domain area. 
Figure 4.4. Effect of a low dose $(50 \mu \mathrm{g})$ of fengycin on liquid disordered (Ld) domain characteristics and distribution of lipid bilayers. Bilayers contained extracted lipids from Pythium sulcatum (blue), Alternaria solani (orange), and Fusarium sambucinum (grey). Fengycin added at the 0 minute (dose 1) and the 40 minute (dose 2) marks. (A) $\mathrm{L}_{d}$ domain count; (B) $\mathrm{L}_{d}$ domain size; (C) $\mathrm{L}_{\mathrm{d}}$ domain area.

Figure 4.5. Effect of a high dose $(500 \mu \mathrm{g})$ of fengycin on liquid ordered (Lo) domain characteristics and distribution of lipid bilayers. Bilayers contained extracted lipids from Pythium sulcatum (blue), Alternaria solani (orange), and Fusarium sambucinum (grey). Fengycin added at the 0 minute mark. (A) $\mathrm{L}_{\mathrm{o}}$ domain count; (B) $\mathrm{L}_{\mathrm{o}}$ domain size; (C) $\mathrm{L}_{\mathrm{o}}$ domain area. 123

Figure 4.6. Effect of a high dose $(500 \mu \mathrm{g})$ of fengycin on liquid disordered (Ld) domain characteristics and distribution of lipid bilayers. Bilayers contained extracted lipids from Pythium sulcatum (blue), Alternaria solani (orange), and Fusarium sambucinum (grey). Fengycin added at the 0 minute mark. (A) $\mathrm{L}_{d}$ domain count; (B) $\mathrm{L}_{d}$ domain size; (C) $\mathrm{L}_{d}$ domain area. 125

\section{Supplementary Materials}

Figure S1. Effect of low doses $(50 \mu \mathrm{g})$ of fengycin on liquid ordered $\left(\mathrm{L}_{\mathrm{o}}\right)$ domain characteristics and distribution of lipid bilayers. Bilayers contained 3:1 DOPC:DPPC with 0 (blue), 3 (orange), 6 (grey), and 12\% (yellow) ergosterol. Fengycin added at the 0 minute (dose 1) and the 40 minute marks (dose 2). A-C. Small domains $(<1 \mu \mathrm{m})$; D-F. Medium domains (1-10 $\mu \mathrm{m})$; G-I. Large domains $(>10 \mu \mathrm{m})$. A, D, G. Relative domain count; B, D, H. Relative domain size; C, F, I. Relative domain area. 148

Figure S2. Effect of low doses $(50 \mu \mathrm{g})$ of fengycin on liquid disordered $\left(\mathrm{L}_{\mathrm{d}}\right)$ domain characteristics and distribution of lipid bilayers. Bilayers contained 3:1 DOPC:DPPC with 0 (blue), 3 (orange), 6 (grey), and 12\% (yellow) ergosterol. Fengycin added at the 0 minute (dose 1) and the 40 minute marks (dose 2). A-C. Small domains $(<1 \mu \mathrm{m})$; D-F. Medium domains (1-10 $\mu \mathrm{m})$; G-I. Large domains $(>10 \mu \mathrm{m})$. A, D, G. Relative domain count; B, D, H. Relative domain size; C, F, I. .. 149 Figure S3. Effect of a high dose $(500 \mu \mathrm{g})$ of fengycin on liquid ordered $\left(\mathrm{L}_{\mathrm{o}}\right)$ domain characteristics and distribution of lipid bilayers. Bilayers contained 3:1 DOPC:DPPC with 0 (blue), 3 (orange), 6 (grey), and 12\% (yellow) ergosterol. Fengycin added at the 0 minute marks. A-C. Small domains 
$(<1 \mu \mathrm{m})$; D-F. Medium domains $(1-10 \mu \mathrm{m})$; G-I. Large domains (>10 $\mu \mathrm{m})$. A, D, G. Relative domain count; B, D, H. Relative domain size; C, F, I. Relative domain area. ......................... 150 Figure S4. Effect of a high dose $(500 \mu \mathrm{g})$ of fengycin on liquid disordered $\left(\mathrm{L}_{\mathrm{d}}\right)$ domain characteristics and distribution of lipid bilayers. Bilayers contained 3:1 DOPC:DPPC with 0 (blue), 3 (orange), 6 (grey), and 12\% (yellow) ergosterol. Fengycin added at the 0 minute marks. A-C. Small domains (<1 $\mu \mathrm{m})$; D-F. Medium domains (1-10 $\mu \mathrm{m})$; G-I. Large domains ( $>10 \mu \mathrm{m})$. A, D, G. Relative domain count; B, D, H. Relative domain size; C, F, I. Relative domain area........ 151 


\section{List of Tables}

\section{Chapter 1}

Table 1.1. Known modes of action of currently available fungicides....................................... 4

Table 1.2. Examples of disease suppression by biocontrol strains of Bacillus subtilis .............. 11

\section{Chapter 4}

Table 4.1. Characteristics of lipids extracted from Alternaria solani, Fusarium sambucinum, and Pythium sulcatum.

129

Table 4.2. Correlation of lipid parameters with microbial tolerance to fengycin 130 


\section{Notations and Abbreviations}
AFM
Atomic force microscopy
$\mathrm{AmpB}$
Amphotericin B
AMPs
Antimicrobial peptides
$\mathrm{A}: \mathrm{Z}$
Anionic: zwitterionic
BODIPY FL-DHPE $\quad N$-(4,4-difluoro-5,7-dimethyl-4-bora-3a,4a-diaza-s-indacene-3-propionyl)- 1,2-dihexadecanoyl-sn-glycero-3-phosphoethanolamine, triethylammonium salt
CL
Cardiolipin
$\operatorname{DilC}_{18}$
1,1'-Dioctadecyl-3,3,3',3'-tetramethylindocarbocyanine perchlorate
DOPC
1,2-dioleoyl-sn-glycero-3-phosphocholine
DOPE
1,2-dioleoyl-sn-glycero-3-phosphoethanloamine
DOPS
1,2-dioleoyl-sn-glycero-3-phospho-L-serine
DPPC
1,2-dipalmitoyl-sn-glycero-3-phoshpocholine
DPPE
1,2-dipalmitoyl-sn-glycero-3-phosphoethanolamine
DPPG
1,2-dipalmitoyl-sn-glycero-3-phospho-(1'-rac-glycerol)
DSC
Differential scanning calorimetry
FAO
Food and Agriculture Organization of the United Nations
FRAC
Fungicide Resistance Action Committee 


\begin{tabular}{|c|c|}
\hline GUVs & Giant unilamellar vesicles \\
\hline ITC & Isothermal titration calorimetry \\
\hline$l_{\alpha}$ & Liquid disordered state \\
\hline$l_{\beta}$ & Solid gel state \\
\hline $\mathrm{L}_{\mathrm{o}}$ & Ordered state \\
\hline $\mathrm{L}_{d}$ & Disordered state \\
\hline LUVs & Large unilamellar vesicles \\
\hline MLVs & Multilamellar vesicles \\
\hline M:P & Monounsaturated: polyunsaturated \\
\hline NBD-PE & $\begin{array}{l}\text { 1,2-Dimyristoyl-sn-glycero-3-phosphoethanolamine- } N \text {-(7-nitro-2-1,3- } \\
\text { benzoxadiazol-4-yl) (ammonium salt) }\end{array}$ \\
\hline $\mathrm{PA}$ & Phosphatidic acid \\
\hline PC & Phosphatidylcholine \\
\hline $\mathrm{PE}$ & Phosphatidylethanolamine \\
\hline PG & Phosphatidylglycerol \\
\hline POPC & 1-Palmitoyl-2-oleoyl-glycero-3-phosphocholine \\
\hline POPE & 1-Palmitoyl-2-oleoyl-sn-glycero-3-phosphoethanolamine \\
\hline POPG & 1-Palmitoyl-2-oleoyl-sn-glycero-3-phospho-(1'-rac-glycerol) \\
\hline PS & Phosphatidylserine \\
\hline
\end{tabular}


$\mathrm{Q}_{0} \mathrm{Is}$

SDHIs

SLBs

SUVs

TIRF

TIRFM

TLC

TR-DHPE

$\mathrm{U}: \mathrm{S}$

$\Delta /$ mol total
Quinone outside inhibitors

Succinate dehydrogenase inhibitors

Supported lipid bilayers

Small unilamellar vesicles

Total internal reflection fluorescence

Total internal reflection fluorescence microscopy

Thin layer chromatography

Texas Red ${ }^{\mathrm{TM}}$ 1,2-dihexadecanoyl-sn-glycero-3-phosphoethanolamine, triethylammonium salt

Unsaturated:saturated

Average unsaturation per mol of total lipid 


\section{Preface}

This preface provides full bibliographical details for each article includes in this thesis, as well as whether the article is reproduced in whole or in part. Use of copyrighted material is acknowledged in this preface. When citing material from this thesis, please cite the article presented in the chapter, if based on a publication.

In accordance with the Integrated Thesis policy of Carleton University, the supervisor (Tyler Avis) and the student (Elisabeth Mantil) confirm that the student was fully involved in setting up and conducting the research, obtaining data and analyzing results, as well as preparing and writing the material presented in this integrated thesis.

The articles:

\section{Chapter 2}

E. Mantil, T. Crippin, A. Ianoul, T.J. Avis, Experimental parameters leading to optimal bilayers for total internal reflection fluorescence microscopy visualization, Microsc. Microanal, 23 (2017) $97-112$.

This article is wholly reproduced and edited for formatting and clarity of presentation. SLB experiments were set up by Elisabeth Mantil, performed by both Elisabeth Mantil and Trinda Crippin, and analyzed by Elisabeth Mantil. Anatoli Ianouli was consulted on for TIRFM analysis. This publication was written by Elisabeth Mantil. 


\section{Chapter 3}

E. Mantil, T. Crippin, T.J. Avis, Domain redistribution within ergosterol-containing model membranes in the presence of the antimicrobial compound fengycin, Manuscript in preparation (2018).

SLB control experiments were set up by Elisabeth Mantil, performed by both Elisabeth Mantil and Trinda Crippin, and analyzed by Elisabeth Mantil. Fengycin trials were set up, performed and analyzed by Elisabeth Mantil. This chapter was written by Elisabeth Mantil (As-yet unpublished).

\section{Chapter 4}

E. Mantil, T. Crippin, T.J. Avis, Supported lipid bilayers using extracted microbial lipids: domain redistribution in the presence of fengycin, Manuscript in preparation (2018).

SLB control and fengycin experiments were set up, performed, and analyzed by Elisabeth Mantil. Microbial lipids were extracted by Trinda Crippin. This chapter was written by Elisabeth Mantil (As-yet unpublished). 
Chapter 1

General Introduction 


\section{Introduction}

\subsection{The agricultural system}

1.1.1. Pathogenic fungi and their impact on crop yield and food spoilage

According to census data collected by the United Nation, the world's population grew at a rate of $1.24 \%$ per year approximately a decade ago. By 2017 , the growth rate had fallen to $1.10 \%$ per year. Nevertheless, the population is projected to increase steadily (Fig. 1.1), reaching 9.8 billion by the year 2050 [1]. Even with the current decline in fertility, and assuming an acceleration of infertility, the global population is expected to continue growing. For example, the fertility rate of Africa has decreased from 5.1 births per woman (2000-2005) to 4.7 (2010-2015), but 26 African countries are projected to at least double in population size by the year 2050 [1]. Increases in populations lead to increases in demand for food crops and land resources.

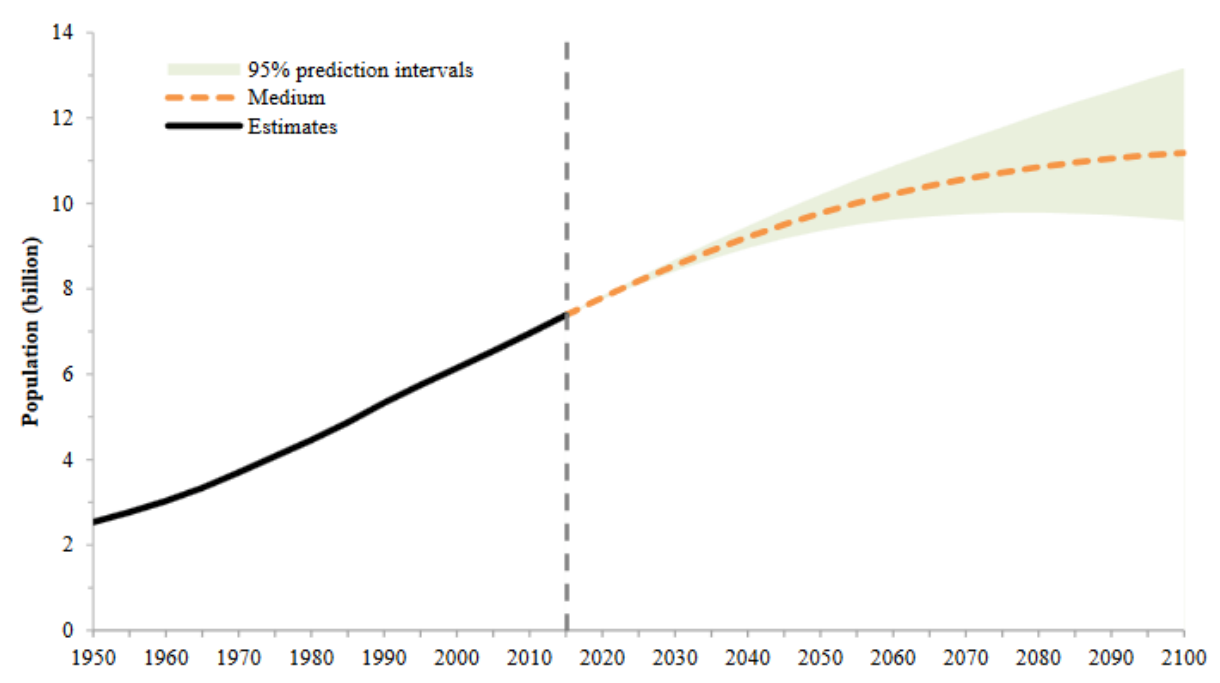

Figure 1.1. Population of the world: estimates, 1950-2015, and mediumvariant projection with 95 per cent prediction intervals, 2015-2100 [1]. 
As the population increases, economic growth is expected to increase at $2.9 \%$ annually, until 2050 , leading to a significant reduction in poverty and malnutrition. While it is expected to be significantly less, there will remain widespread economic depression and malnutrition. These will influence the future demand for food. Demand for cereal crops is projected to reach 3 billion tonnes by 2050, up from 2.1 billion used in 2009. Overall, food production will need to increase by $70 \%$ in order to meet the needs of the growing population [2]. These crops are needed not only for human consumption, but as animal feed. This is greatly influenced by the use of biofuels and demand for food products associated with higher incomes (consumption of animal products and vegetable oils) [3].

In the future, $90 \%$ of the crops to be produced, are expected to be produced on arable land currently available, expecting increases in crop yields and cropping intensity within the land already in use. Arable land is expected to increase by only $5 \%$, with primary expansion happening in developing countries. While an expansion in arable land is predicted in developing countries, an $8 \%$ decrease in arable land is expected in developed countries, as populations expand [3]. There is therefore a need to increase crop yield, while using less land and resources. This includes the necessity to effectively control crop pests and pathogens to reduce the associated food loss. Crop losses not only increase risk of malnutrition and lower food availability for the world population, they also lead to wasting of natural resources such as land, water and energy inputs, and create unnecessary $\mathrm{CO}_{2}$ emissions [4].

According to the FAO [4], approximately one third of all food products are lost before consumption. One contributing factor is the destruction of crops pre- and post-harvest, which can be influenced by abiotic (e.g., water, soil quality, energy input) and biotic factors (e.g., disease agents, insects) [5]. Pathogenic attacks can be attributed to infections produced by microorganisms 
such as bacteria, fungi, oomycetes, and viruses. Crop loss due to pathogens is responsible for approximately $20 \%$ of the global harvest. Another $10 \%$ crop loss can be attributed to pathogenic infections post-harvest (i.e., food spoilage). Of these pathogens, fungi and oomycetes are the most prominent threat [5]. Controlling pathogenic outbreaks in crops, is a key step in ensuring food stability for future population growth.

\subsubsection{Current control methods and potential drawbacks}

Controlling pathogenic outbreaks in crops is a necessity and the application of fungicides will continue to play a major role in controlling such outbreaks. The application of synthetic chemicals rose quickly between the 1940s and 1950s, before the introduction of regulations in the 1960 's, to control the quantity of chemicals applied to crops [6]. Organizations such as the Fungicide Action Resistance Committee (FRAC) has provided data on current fungicides available (Table 1.1), the mode of action they possess, mechanisms of resistance, and their potential risk of resistance [7]. This information is a great resource for the R \& D of new fungicides.

Table 1.1. Known modes of action of currently available fungicides. Adapted from FRAC Code List $^{\circledR} 2018$ [7].

\begin{tabular}{|c|c|c|}
\hline Mode of action & Target site & Group \\
\hline \multirow{4}{*}{$\begin{array}{l}\text { Nucleic acid } \\
\text { metabolism }\end{array}$} & RNA polymerase I & Phenylamides \\
\hline & Adenosin-deaminase & Hydroxyl-(2-amino-)pyrimidines \\
\hline & DNA/RNA synthesis & Heteroaromatics \\
\hline & DNA topoisomerase type II & Carboxylic acids \\
\hline \multirow[t]{2}{*}{$\begin{array}{l}\text { Cytoskeleton and } \\
\text { motor protein }\end{array}$} & $\beta$-tubulin assembly in mitosis & $\begin{array}{l}\text { Methyl benzimidazoles carbamates, N-phenyl } \\
\text { carbamates, benzamides, thiazole } \\
\text { carboxamide }\end{array}$ \\
\hline & Cell division & Phenylureas \\
\hline
\end{tabular}




\begin{tabular}{|c|c|c|}
\hline & Delocalisation of spectrin-like proteins & Benzamides \\
\hline & Actin/myosin/fimbrin & Cyanoacrylates, aryl-phenyl-ketones \\
\hline \multirow[t]{6}{*}{ Cellular respiration } & Complex I - NADH oxido-reductase & $\begin{array}{l}\text { Pyrimidinamines, pyrazole-MET1, } \\
\text { quinazoline }\end{array}$ \\
\hline & Complex II - succinate dehydrogenase & Succinate dehydrogenase inhibitors \\
\hline & $\begin{array}{l}\text { Complex III - cytochrome bc1 (i) at Qi } \\
\text { site and (ii) at Qo site }\end{array}$ & $\begin{array}{l}\text { (i) Quinone inside inhibitors, and (ii) quinone } \\
\text { outside inhibitors }\end{array}$ \\
\hline & Uncouplers of oxidative phosphorylation & N/A \\
\hline & $\begin{array}{l}\text { Inhibitors of oxidative phosphorylation, } \\
\text { ATP synthase }\end{array}$ & Organo tin compounds \\
\hline & ATP transport & Thiophenecarboxamides \\
\hline \multirow{4}{*}{$\begin{array}{l}\text { Amino acid and } \\
\text { protein synthesis }\end{array}$} & Methionine biosynthesis & Anilinopyrimidines \\
\hline & $\begin{array}{l}\text { Protein synthesis (ribosome initiation } \\
\text { step) }\end{array}$ & $\begin{array}{l}\text { Hexopyranosyl antibiotic, glucopyranosyl } \\
\text { antibiotic }\end{array}$ \\
\hline & $\begin{array}{l}\text { Protein synthesis (ribosome elongation } \\
\text { step) }\end{array}$ & Tetracycline antibiotic \\
\hline & $\begin{array}{l}\text { Protein synthesis (ribosome termination } \\
\text { step) }\end{array}$ & Enopyranuronic acid antibiotic \\
\hline \multirow[t]{2}{*}{ Signal transduction } & Signal transduction & Azanaphthalenes \\
\hline & $\begin{array}{l}\text { MAP/histidine kinase in osmotic signal } \\
\text { transduction: (i) os-1, HOG1, and (ii) os- } \\
\text { 1, Daf1 }\end{array}$ & (i) Phenylpyrroles, and (ii) dicarboxamides \\
\hline \multirow{7}{*}{$\begin{array}{l}\text { Lipid synthesis or } \\
\text { transport/membrane } \\
\text { integrity or function }\end{array}$} & $\begin{array}{l}\text { Phospholipid biosynthesis, } \\
\text { methyltransferase }\end{array}$ & Phosphorothiolates, dithiolanes \\
\hline & Cell peroxidation & $\begin{array}{l}\text { Aromatic hydrocarbons, chlorophenyls, } \\
\text { nitroanilines, heteroaromatics }\end{array}$ \\
\hline & Cell membrane permeability, fatty acids & Carbamates \\
\hline & $\begin{array}{l}\text { Microbial disruptors of pathogen cell } \\
\text { membranes }\end{array}$ & Microbial \\
\hline & Cell membrane disruption & Plant extracts \\
\hline & Ergosterol binding & Polyene \\
\hline & Lipid homeostasis and transfer/storage & $\begin{array}{l}\text { Oxysterol binding protein homologue } \\
\text { inhibition }\end{array}$ \\
\hline \multirow{4}{*}{$\begin{array}{l}\text { Sterol biosynthesis } \\
\text { in membranes }\end{array}$} & C14-Demethylase (erg11/cyp51) & Demethylation inhibitors \\
\hline & $\begin{array}{l}\Delta^{14} \text {-Reductase and } \Delta^{8} \rightarrow \Delta^{7} \text {-isomerase (erg } \\
24 \text {, erg2) }\end{array}$ & Amines \\
\hline & $\begin{array}{l}\text { 3-Keto reductase, C-4 demethylation } \\
\text { (erg27) }\end{array}$ & Ketoreductase inhibitors \\
\hline & Squalene-epoxidase (erg1) & \\
\hline \multirow{2}{*}{$\begin{array}{l}\text { Cell wall } \\
\text { biosynthesis }\end{array}$} & Chitin synthase & Polyoxins \\
\hline & Cellulose synthase & Carboxylic acid amides \\
\hline
\end{tabular}




\begin{tabular}{|c|c|c|}
\hline \multirow{3}{*}{$\begin{array}{l}\text { Melanin synthesis } \\
\text { in cell wall }\end{array}$} & Reductase & Melanin biosynthesis inhibitors - reductase \\
\hline & Dehydratase & Melanin biosynthesis inhibitors - dehydratase \\
\hline & Polyketide synthase & Melanin biosynthesis inhibitors - synthase \\
\hline \multirow[t]{5}{*}{$\begin{array}{l}\text { Host plant defence } \\
\text { induction }\end{array}$} & Salicylate-related & $\begin{array}{l}\text { Benzothiadiazole, benzisothiazole, } \\
\text { thiadiazolecarboxamide }\end{array}$ \\
\hline & Polysaccharide elicitors & Natural compound \\
\hline & Anthraquinone elicitors & Plant extract \\
\hline & Microbial elicitors & Microbial \\
\hline & Phosphonates & Phosphonates \\
\hline Unknown & Unknown & $\begin{array}{l}\text { E.g., phthalamic acids, pyridazinones, } \\
\text { guanidines, tetrazolyloxime }\end{array}$ \\
\hline Multisite & Multisite contact & $\begin{array}{l}\text { E.g., dithiocarbamates, chloronitriles, } \\
\text { sulfamides, quinoxalines }\end{array}$ \\
\hline
\end{tabular}

Fungicide agents such as aromatic hydrocarbons, organomercury compounds, demethylation inhibitors and succinate dehydrogenase inhibitors (SDHIs) have been used [8]. Fungal species have developed resistance to these compounds making control of fungal outbreaks difficult. Single-site fungicides are most vulnerable to resistance due to genetic plasticity and rapid replication of the microorganism, in combination with over-application of the compound [8,9]. Multi-site fungicides are more difficult to overcome due to multiple genes used in the resistance process [8]. Reducing overuse and/or misuse of antimicrobial agents can also aid in deterring or slowing the development on resistance [9].

Botrytis cinerea, one of the most economically important fungal pathogens [10] and the causal agent of grey mould, is an excellent example of a microorganism having developed fungicide resistance. $B$. cinerea has revealed resistance against multiple fungicides including benzimidazoles, dicarboximides, anilinopyrimidines, quinone outside inhibitors (QoIs)/strobilurins, and SDHIs. Resistance has been found on multiple crops, including berries, tomatoes and grapes [11-15]. With increasing levels of fungicide resistance, there is an increasing 
need to develop new antifungal agents. Unfortunately, with increasing regulatory requirements (toxicology, metabolic and environmental impact), it has become more expensive to produce and evaluate novel fungicides. As a result, very few companies are able to invest in the R \& D of new products [6].

Moving forward, the development of new antifungal treatments is of the utmost importance. There is the need to find antimicrobial agents with a more generalized mode of action (broad spectrum), limiting the potential for microbial resistance, preferably antifungal agents that can be applied at low doses and possess low ecological impact [5]. The use of biocontrol agents and biotechnology will play a large role in the development of antifungal treatment with limited risk of resistance.

\subsubsection{Use of biocontrol agents}

There has been a strong push from consumers to reduce the application of synthetic pesticides to limit the environmental and human health impact of such compounds. While new synthetic pesticides can take up to 10 years to be developed and approved, biopesticides take on average three to six years and are 10-fold cheaper [16]. This has made the use of biocontrol agents, also known as biopesticides, an attractive option. Currently biopesticides only account for approximately $2 \%$ of the market.

For a biocontrol agent to be successful, its ability to protect against multiple pathogens is necessary. The biocontrol treatment can be a microorganism itself (i.e., a biocontrol agent) or the metabolites it produces. To ensure efficacy of the application of a microorganism, the target pathogen and its environmental niche needs to be evaluated. The mechanisms of action and modes

of application are also key in the success of the microorganism. In some cases, the metabolite 
produced by the microorganism of interest is more effective than the application of the microorganism. For example, the lipopeptides produced by Bacillus subtilis have shown greater activity against fungal diseases in the field, than the bacteria when applied to crops [17].

The application of biopesticides has been deterred by the limited knowledge on exact mechanisms of action and weak policy frameworks. The development of regulations to ensure quality of products and control practices, as well as research evaluating their production and application can aid in the commercialization process [18].

\subsection{Biocontrol mechanisms of action}

\subsubsection{Fungal membranes - form and function}

Cellular membranes protect the inner contents of a cell while still maintaining semipermeability to aid in the transfer of molecules in and out of the cell. It is essential for the proper growth and survival of all cells. In general, cellular membranes are composed of a fluid lipid bilayer with proteins dispersed throughout [19]. Lipid content of fungi is highly variable between species and is impacted by the growth conditions of the fungi. In general, the lipids found in fungi provide the same function as those found in other organisms. Phospholipids are the structural backbone of the cellular membrane, and sterols are a major structural component of cellular membranes. Sterols contribute to the permeability of the cellular membrane by control bilayer thickness, lipid packing and rotational freedom on the phospholipids in the membrane [20]. They are also a key component in the activity of polyene antibiotics. For example, the activity of amphotericin $\mathrm{B}(\mathrm{AmpB})$ is directly related to the ergosterol content in fungal membranes. It was previous determined that $\mathrm{AmpB}$ binds to ergosterol within the cellular membrane to induce pore 
formation. Therefore, absence of ergosterol leads to loss of AmpB acting on cell membranes [21, 22]. More recently, AmpB has been shown act as a sterol sponge. AmpB forms large extramembranous aggregates with extra ergosterol from the membrane. This creates membranes deficient in ergosterol, inhibiting all the cellular processes dependent on the presence of ergosterol [23]. The overall composition of a fungal membrane has a large influence on the capabilities of current synthetic antifungal agents, as well as biocontrol agents currently being evaluated.

\subsubsection{General modes of action}

Biocontrol agents can produce antimicrobial effects in a variety of manners. One of the first, more obvious methods by which the microorganisms display their antimicrobial effects is by outcompeting the pathogens for nutrients and space within the environment $[24,25]$. This activity is highly dependent on the concentration of the antagonist present. While competing for nutrients is effective, it alone will not produce a successful biocontrol treatment. For a biocontrol agent to be successful, it requires a broad-spectrum of activity.

While outcompeting the growth of a pathogen is most effective, antibiosis is a close second for ideal suppression of pathogenic growth. Antibiosis is the mode of action by which antagonists produce antimicrobial compounds, detrimental to the growth of pathogens.

Other modes of action include parasitism and induced resistance [24, 25]. Parasitism is the direct interaction of the microorganism with pathogen tissue. This has been illustrated in Trichoderma spp. which parasitize the hyphae of other fungi by wrapping around its host, occasionally penetrating the host by partial degradation of the cell wall [26, 27]. Antagonistic microbes can also promote defense mechanisms of the host, such as the production of antifungal 
compounds or physical structure impeding pathogen ingress or expansion. These effects can lead to stimulation of wound healing in host plants and induced pathogen resistance in the host [25].

\subsection{Bacillus subtilis and its metabolites}

\subsubsection{Biocontrol spectrum}

The antagonistic bacterium Bacillus subtilis has been shown on numerous occasions to reduce or eliminate the growth of pathogens on crops. Some of the diseases $B$. subtilis is effective in suppressing are listed in Table 1.2 below. While exhibiting fungal disease reduction on crops, B. subtilis has also exhibited these antifungal capabilities via in vitro on a number of other fungi [28-30]. 
Table 1.2. Examples of disease suppression by biocontrol strains of Bacillus subtilis

\begin{tabular}{llll}
\hline Disease & Pathogen & Crop & Reference \\
\hline Green mould & Penicillium digitatum & Citrus & {$[30]$} \\
Sour rot & Geotricum candidum & Citrus & {$[30]$} \\
Stem end rot & Alternaria citri, & Avocado, citrus & {$[30,31]$} \\
& Botryodiplodia theobromae, & & \\
& Phomopsis citri. & & \\
Brown rot & Lasiodiplodia theobromae & Apricot, & nectarine, \\
& & peach, plum & \\
Botrytis rot/gray & Botrytis cinerea & Cherry, strawberries & {$[33-35]$} \\
mould & & & {$[36]$} \\
Downy blight & Peronophythora litchi & Litchi & {$[37]$} \\
Postharvest rot & Aspergillus niger, B. & Yams & \\
& theobromae, Penicillium & & {$[38]$} \\
Sclerotinia stem rot & Sclerotinia sclerotiorum & Rape & \\
\hline
\end{tabular}

\subsubsection{Metabolites produced}

The antimicrobial capabilities of $B$. subtilis are mainly attributed to its production of three lipopeptides families: surfactin, iturin, and fengycin. The application of these compounds in biotechnology has great potential due to their surfactant properties. Each family of molecules is 
composed of a peptide ring containing seven (surfactin and iturin) or ten (fengycin) amino acid residues, and a $\beta$-hydroxy (surfactin and fengycin) or $\beta$-amino (iturin) fatty acid [40-42]. The length of the fatty acid varies for each lipopeptide family, leading to a series of isomers within

each family. In general, surfactins contain fatty acids ranging from 13-16 carbons, iturins from 1417, and fengycins from 14-18 [40-42]. Their structure diversity influences their overall efficacy as antimicrobial agents. Although reports vary considerably concerning the overall efficacy of these families, it is general reported that surfactins are mostly effective against bacteria [43, 44], whereas iturin and fengycin are mostly considered antifungal compounds [45, 46]. However, fengycins are less hemolytic than iturins [44], thereby showing increased interest as an antimycotic agent.

\subsection{Fengycin}

\subsubsection{Structure}

The general structure of fengycin $\mathrm{A}$ is illustrated in Fig. 1.2. Variation in amino acid residues differentiates fengycin homologs and isomers $[42,47]$ as described in Figure 1.2. The $\beta$ hydroxy fatty acid is variable in length (generally ranging from $\mathrm{C}_{14}$ to $\mathrm{C}_{18}$ ) can be saturated or unsaturated. Unsaturations have been noted at $\mathrm{C}_{2}, \mathrm{C}_{3}$, and $\mathrm{C}_{13}$ in the acyl chains [41]. 


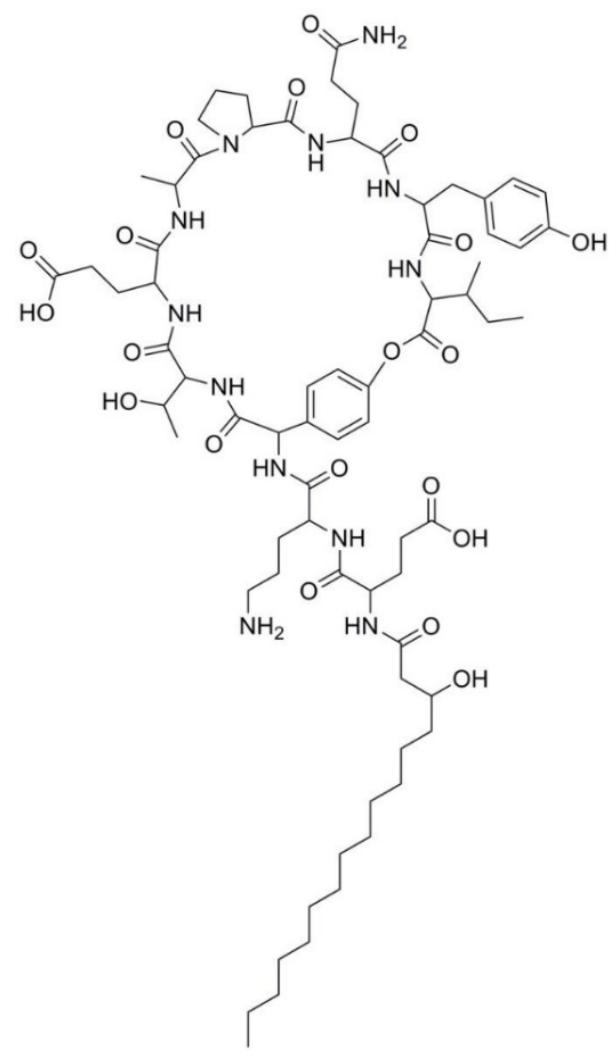

Fengycin homolog

Amino acid residue sequence

Fengycin A

L-Glu-D-Orn-D-Tyr-D-aThr-L-Glu-D-Ala-

L-Pro-L-Glu-L-Tyr-L-Ile

Fengycin B

L-Glu-D-Orn-D-Tyr-D-aThr-L-Glu-D-Val-

L-Pro-L-Glu-L-Tyr-L-Ile

Plipstatin A

L-Glu-D-Orn-L-Tyr-D-aThr-L-Glu-D-Ala-

L-Pro-L-Glu-D-Tyr-L-Ile

Plipstatin B

L-Glu-D-Orn-L-Tyr-D-aThr-L-Glu-D-Val-

L-Pro-L-Glu-D-Tyr-L-Ile

Figure 1.2. General structure of the fengycin A molecule. Table depicts the variances in amino acid residues of the cyclic peptide. 


\subsubsection{Mechanism of action}

It is known that fengycin's fungicidal capabilities are due to its interactions with the lipid layers, altering the structure of the cellular membrane and reducing its barrier capabilities. Fengycin is noted to act in an all-or-none, rather than a graded fashion, but the overall mechanism(s) behind this are not fully understood [48].

While many bioassays have been carried out to study the direct antifungal activity of fengycin on fungal growth, such as that completed by Vanittanakom et al. [49], less has been completed on the specific interaction of fengycin with fungal membranes. Of this work, several techniques have been used to help determine the specific actions of fengycin with cellular membranes, including the use of artificial models. These artificial models consist of lipid monolayers, supported lipid bilayers, and liposomes (uni- and multi-lamellar vesicles).

\subsubsection{Lipid monolayers}

Experiments evaluating fengycin activity on monolayers has shown its preference for fatty acyl chain insertion into the fluid phase, leaving the cyclic peptide moiety to sit on the top of the lipid layer. Deleu et al. [50] evaluated the insertion of fengycin into dipalmitoylphosphatidylcholine (DPPC) monolayers via surface pressure isotherms using a Langmuir trough, followed by imaging of the monolayers using a Brewster angle microscope. At low concentrations of fengycin (molar fraction of 0.1 to 0.5 ), it was seen to be partially miscible with the DPPC monolayer, interfering with the close packing of the DPPC molecules. Differential scanning calorimetry (DSC) supported these findings [46]. This fluidizing effect prevents DPPC from transferring into a gel state, but does promote the formation of DPPC-rich and fengycin-rich 
domains $[46,50,51]$. At higher concentrations (a molar fraction of 0.66 ), fengycin completely dissolves the ordered phases and disorganized the monolayer. From these results, it is suggested that at low concentrations, fengycin inserts itself into the membrane and forms pores. At higher concentrations, fengycin self-assembles forming aggregates and solubilizes the membranes in a detergent-like manner [50].

Fengycin contains an ornithine and two glutamic acid residues, which can be protonated or deprotonated, depending on its environment, impacting its activity with a membrane [52]. DPPC and fengycin are more miscible when fengycin is protonated (net charge $=$ zero) at a $\mathrm{pH}$ of 2 , than at a $\mathrm{pH}$ of 7 when it has a negative net charge (-1). The absence of a net charge eases interactions between the fengycin molecules and the zwitterionic head groups of DPPC. When carrying out compression isotherms, it was seen that, at a $\mathrm{pH}$ of 2 , fengycin was more strongly and rapidly pushed out of DPPC bilayers. Fengycin molecules were also expelled from the DPPC monolayer at a $\mathrm{pH}$ of 7 , but in a more progressive manner [52].

\subsubsection{Supported lipid bilayers}

As mentioned previously, fengycin preferentially inserts into the fluid state of membranes [46]. While it is still able to insert into gel phase lipids, it is not completely embedded in the bilayer and protrudes out of the upper lipid leaflet, thickening supported lipid bilayers [46, 51]. Lipids that take on a gel phase formation deter the insertion of fengycin due to their close lipid packing, even at higher concentrations of fengycin [46]. At low concentrations of fengycin (below $180 \mu \mathrm{M}$ ), fengycin readily inserts itself into 1,2-dioleoyl-sn-glycero-3-phosphocholine (DOPC) bilayers without contributing to the bilayer thickness. At concentrations of $180 \mu \mathrm{M}$, a reduction in bilayer thickness can be measured before reaching zero, indicating complete dissolution of the bilayer. 
This is attributed to the formation of fengycin aggregates inducing a positive curvature on the membrane, leading to distortion of the membrane $[46,51,53]$.

While fengycin does insert directly into the bilayer, it would interact primarily with the lipid headgroups [51]. Generally speaking, the phosphates and hydroxyls in the lipid head groups are attracted to the ornithine residue of fengycin, which is positively charged [53]. In molecular dynamic simulations, fengycin has shown preference for 1- palmitoyl-2-oleoyl-sn-glycero-3phosphoglycerol (POPG) over 1- palmitoyl-2-oleoyl-sn-glycero-3-phosphoethanolamine (POPE) in binary bilayers. For POPE and fengycin to interact, the lipid would need to adjust its conical shape to accommodate the insertion of fengycin, which is highly unfavourable [51]. Molecular dynamic simulations carried out by Sur et al. [53], noted fengycin having higher binding affinity to PE and is most likely due to the interaction of the negatively charged glutamic acid residues in fengycin's peptide ring with the positively charged amine group found on PE [53]. Overall, fengycin aggregates appear to be more stable in bilayers composed of PC than of binary bilayers composed of PE and PG $[51,53]$. This stability can be attributed to the lack of side chain interactions occurring between the PC lipid head group [53].

\subsubsection{Liposomes}

Antimicrobial activity is influenced by the homolog present. In a liposome leakage assay using large unilamellar vesicles (LUVs), the plipstatin A1 homolog was incorporated into the lipid bilayer as the concentration of plipstatin A1 was increased in their external environment. Agrastatin, a fengycin-type lipopeptide, did not incorporate into the bilayer even with increasing external concentrations. Increases in concentrations led to formation of agrastatin crystals in the external environment of the LUVs [48]. 
Previous work completed by Wise et al. [54] noted a strong positive correlation between ergosterol content and fengycin tolerance amongst molds. Fiedler et al. [55] investigated this further using a liposome leakage assay. Simple binary liposomes of 1- palmitoyl-2-oleoyl-snglycero-3-phosphocholine (POPC) and either cholesterol or ergosterol, at concentrations of 10$30 \%$, were evaluated in the presence of fengycin. Overall, both sterols inhibited leakage caused by fengycin. As the content of cholesterol increased, fengycin activity decreased, essentially fully inhibiting the activity of fengycin. The presence of ergosterol produced the same effect, albeit to a lesser extent. Fiedler et al. [55] also noted reduced sensitivity with POPE incorporated into the membrane. Interestingly, when the POPE content was increase from 20 to $40 \%$ in POPC liposomes, fengycin inhibitory activity was reduced, but in liposomes of POPG with $60 \%$ POPE, it exhibited a larger inhibitory effect. This was attributed to the negative curvature produced by PE lipids [55].

Recent work carried out on multilamellar vesicles (MLVs) noted the dehydration of PC polar head groups after the application of fengycin [56]. This dehydration effect results in the loss of hydrogen bonding capabilities, reducing water interactions with the phospholipid head group (such as the oxygens in the phosphate group) leading to a loss of barrier function in the cell membrane due to a reduction in membrane fluidity.

Overall, the lipid composition of the cellular membrane dictates fengycin interactions and its antimicrobial capabilities. The exact mechanisms behind this are still not completely understood, but is thought to directly affect fengycin ability to induce curvature in the membrane [51]. Tight packing of lipids leads to a shallow insertion of fengycin's fatty acyl chain, compared to that of fluid lipid phases $[46,51]$. Single fengycin molecules insert into the membrane creating little disturbance. Fengycin aggregates lead to changes in the bilayer thickness, leading to 
dissolution of the bilayer $[46,51,53,57]$. Because of its poor miscibility, fengycin may cause local disruption in bilayers, which would not have an impact on the bulk properties of the bilayer until higher concentrations are reached. Fengycin-rich clusters mimic detergent-rich clusters and solubilize the membrane $[51,53]$.

\subsection{Analysis of membrane interactions}

Membrane interactions can be analyzed through a variety of biophysical tools, each provided slightly different views on the interactions of molecules with model membranes. Ultimately, these methods complement one another. As described above, model membranes can take on the form of a monolayer, a supported lipid bilayer or a liposome. Each of these model membrane types allow mimicking of natural membranes, which can reveal lipid phase separation and general lipid arrangement. Each model carries its own advantages and disadvantages.

Monolayers formed on the air-water interface of a Langmuir trough are only half of a

bilayer. They are considered to mimic the interactions of a molecules with the outer leaflet of a lipid bilayer. Being built on a Langmuir trough enables full control of the packing of the lipids, subphase composition ( $\mathrm{pH}$ and ionic strength) and the temperature parameters. Monolayers are evaluated via compression isotherms, giving information on the physical state of the lipids (conformational order). Conformational changes in monolayers composed of more than one lipid type can provide information on mixing behaviour (miscibility), and the potential for a compound to induce phase separations. The Langmuir trough can be combined with Brewster angle microscopy, a fluorescent microscopy technique, to visualize such phase separations $[46,58]$. Brewster angle microscopy allows visualization of monolayers on a micrometer scale. To enhance the visualization of monolayers, they can be transfer to a solid support using the Langmuir- 
Blodgett technique and imaged using an atomic force microscope (AFM). An AFM works on a nanometer scale and provides topographical information on the monolayer [59-61]. Unfortunately, transfer of the monolayer to a solid support can influence the organization of the lipids and must be taken into consideration when evaluating the monolayer. Monolayers in the Langmuir trough can also be analyzed using polarized infrared spectroscopy and ellipsometry to structural information on the lipids such as their orientation and conformation within the monolayer [62].

While monolayers are useful, they do not take into consideration the influence of a second phospholipid layer on phase separation and organization. Bilayers can be prepared and analyzed in vesicle form or as supported lipid bilayers bound to a solid support. Depending on the vesicle preparation techniques used, different bilayer structures can be obtained: MLVs (vortexing), giant unilamellar vesicles (GUVs) (gentle hydration or electroformation), LUVs (extrusion through a porous membrane), and small unilamellar vesicles (SUVs) (sonication) [63, 64]. Vesicles are advantageous when evaluating membrane fusion phenomenon and adhesion, unfortunately over time vesicles may aggregate and fuse together. Controlling the external environment of the vesicles is important to maintain their stability. The presence of two lipid leaflets can cause orientation in a manner similar to biological membranes, constituting a more "natural" biological membrane than a monolayer [63]. Vesicles are most commonly viewed with fluorescence and confocal microscopy. DSC and isothermal titration calorimetry (ITC) experiments are carried out on vesicle formations to evaluate phase transitions of the lipids and can be applied to the influence of compounds on phase transitions within the bilayers [46, 56, 58, 62].

SLBs are more stable than vesicles and allow for some control of lipid asymmetry within the bilayer. SLBs can be prepared using the Langmuir Blodgett technique or lipid vesicle fusion $[61,65]$. With the bilayer attached to a solid support (glass, mica or silicon dioxide), they can be 
more easily characterized with sensitive techniques. SLBs are commonly used to evaluate interactions of drugs with model membranes, looking at the phase behaviour and organization of molecules in the bilayer. These characteristics can be analyzed using AFM, optical ellipsometry, X-ray reflectivity, neutron reflectivity, and fluorescence microscopy (e.g., total internal reflection fluorescence (TIRF) microscopy) [58, 62]. TIRF microscopy excites fluorophores near a solid support (within $100 \mathrm{~nm}$ ), in an aqueous environment, via an evanescent wave. This limits excitation fluorescence from sample background (out of focus planes). Lateral movement of lipids can also be evaluated with TIRFM through rapid photobleaching of sample section, and quantitatively measuring the rate of recovery of fluorescence in a process known as fluorescence recovery after photobleaching (FRAP).

\subsection{Hypothesis and objectives}

While work has been completed showing the efficacy of fengycin as an antifungal agent in bioassays, and some work has been focused on the mechanism behind fengycin's activity, there still does not exist a clear mechanism of action for fengycin. Direct visualization of damage caused by fengycin has been captured in images of fungal hyphae confirming membrane impairment [66, 67], along with measurements of membrane permeability $[30,55]$. Work has been completed on the effects of fengycin in simple model membranes showing that fengycin most likely acts by inserting its fatty acyl chain into the hydrophobic core of the lipid bilayer, with its cyclic peptide ring sitting on top of the outer leaflet. While the insertion of a single fengycin molecule has minor effects on the local lipids, fengycin aggregates lead to the dissolution of the bilayer $[46,51,53$, 57]. More recent work has started to indicate that lipid type and bilayer composition significantly 
impact the efficacy of fengycin $[54,55]$. Among these components, the presence of ergosterol seems to greatly influences the efficacy of fengycin $[51,54,55]$.

The present work studied the possible effects of fengycin on membrane domain characteristics using TIRF microscopy. First, a protocol for producing SLBs of a consistent nature

was developed for use in experiments with TIRF microscopy. Secondly, these SLBs were used to determine lipid phase domain characteristics of simple binary bilayers in the presence of ergosterol and fengycin. Finally, domain characterization of SLBs composed of lipids extracted from different plant pathogenic molds were evaluated with particular interest in the lipid composition of these extracts and their influence on phase separations in the bilayer in the presence of fengycin.

\subsubsection{Hypotheses}

(1) Bilayers containing higher levels of ergosterol are more tolerant to fengycin-induced domain distribution changes

(2) Increased presence of saturated phospholipids will increase tolerance within the SLB to fengycin by producing a higher number of ordered lipid domains, reducing fengycin's ability to insert into the bilayer.

\subsubsection{Objectives}

(1) To determine an effective protocol for the preparation of SLBs producing bilayers containing an even and consistent distribution of phospholipids and TR-DHPE.

(2) To evaluate the effects of ergosterol content on lipid domain distribution in bilayers exposed to fengycin. 
(3) To determine the effects of lipid components on domain redistribution in fengycinexposed SLBs composed of complex lipids extracts from plant pathogens.

\subsection{References}

[1] United Nations, World population prospects: the 2017 revision, key findings and the advance tables, in: P.D. Department of Economic and Social Affairs (Ed.), United Nations, New York, 2017.

[2] D. Kumar, P. Kalita, Reducing postharvest losses during storage of grain crops to strengthen food security in developing countries, Foods, 6 (2017).

[3] FAO, Global agriculture towards 2050, Food and Agriculture Organization of the United Nations, Rome, 2011.

[4] FAO, Global food losses and food waste - extent, causes, and prevention, Food and Agriculture Organization of the United Nations, Rome, 2011.

[5] D.P. Bebber, S.J. Gurr, Crop-destroying fungal and oomycete pathogens challenge food security, Fungal Genetics and Biology, 74 (2015) 62-64.

[6] P.E. Russell, A century of fungicide evolution, J Agric Sci, 143 (2005) 11-25.

[7] F.R.A. Committee, FRAC Code List $\left(\mathcal{C}^{*} 2018\right.$ : Fungicides sorted by mode of action (including FRAC Code numbering), 2018.

[8] J.A. Lucas, N.J. Hawkins, B.A. Fraaije, Chapter Two - The evolution of fungicide resistance, in: S. Sariaslani, G.M. Gadd (Eds.) Adv Appl Microbiol, Academic Press 2015, pp. 29-92.

[9] E.P.C. Lai, Z. Iqbal, T.J. Avis, Combating antimicrobial resistance in foodborne microorganisms, J Food Prot, 79 (2016) 321-336.

[10] R. Dean, L. Van Kan Jan A, A. Pretorius Zacharias, E. Hammond-Kosack Kim, A. Di Pietro, D. Spanu Pietro, J. Rudd Jason, M. Dickman, R. Kahmann, J. Ellis, D. Foster Gary, The top 10 fungal pathogens in molecular plant pathology, Mol Plant Path, 13 (2012) 414-430.

[11] R.W.S. Weber, Resistance of Botrytis cinerea to multiple fungicides in Northern German small-fruit production, Plant Dis, 95 (2011) 1263-1269. 
[12] S. Konstantinou, T. Veloukas, M. Leroch, G. Menexes, M. Hahn, G. Karaoglanidis, Population structure, fungicide resistance profile, and $\mathrm{sdhB}$ mutation frequency of Botrytis cinerea from strawberry and greenhouse-grown tomato in Greece, Plant Dis, 99 (2014) 240-248.

[13] S. Rupp, R.W.S. Weber, D. Rieger, P. Detzel, M. Hahn, Spread of Botrytis cinerea strains with multiple fungicide resistance in German horticulture, Front Microbiol, 7 (2016) 2075.

[14] S. Rupp, C. Plesken, S. Rumsey, M. Dowling, G. Schnabel, R.W.S. Weber, M. Hahn, Botrytis fragariae, a new species causing gray mold on strawberries, shows high frequencies of specific and efflux-based fungicide resistance, Appl Environ Microbiol, 83 (2017) e00269-00217.

[15] A. Panebianco, I. Castello, G. Cirvilleri, G. Perrone, F. Epifani, M. Ferrara, G. Polizzi, D.R. Walters, A. Vitale, Detection of Botrytis cinerea field isolates with multiple fungicide resistance from table grape in Sicily, Crop Prot, 77 (2015) 65-73.

[16] J. Popp, K. Pető, J. Nagy, Pesticide productivity and food security. A review, Agron Sustain Dev, 33 (2013) 243-255.

[17] T. Glare, J. Caradus, W. Gelernter, T. Jackson, N. Keyhani, J. Köhl, P. Marrone, L. Morin, A. Stewart, Have biopesticides come of age?, Trends Biotechnol, 30 (2012) 250-258.

[18] S. Kumar, A. Singh, Biopesticides: Present Status and the Future Prospects, J Fertil Pest, 6 (2015) 1-2.

[19] S.J. Singer, G.L. Nicolson, The fluid mosaic model of the structure of cell membranes, Science, 175 (1972) 720-731.

[20] J. Weete, Fungal lipid biochemistry: distribution and metabolism, Springer Science \& Business Media 2012.

[21] K.C. Gray, D.S. Palacios, I. Dailey, M.M. Endo, B.E. Uno, B.C. Wilcock, M.D. Burke, Amphotericin primarily kills yeast by simply binding ergosterol, Proc. Natl. Acad. Sci, 109 (2012) 2234.

[22] S.M. Hammond, 3 Biological activity of polyene antibiotics, in: G.P. Ellis, G.B. West (Eds.) Progress in Medicinal Chemistry, Elsevier1977, pp. 105-179.

[23] T.M. Anderson, M.C. Clay, A.G. Cioffi, K.A. Diaz, G.S. Hisao, M.D. Tuttle, A.J. Nieuwkoop, G. Comellas, N. Maryum, S. Wang, B.E. Uno, E.L. Wildeman, T. Gonen, C.M. Rienstra, M.D. Burke, Amphotericin forms an extramembranous and fungicidal sterol sponge, Nat Chem Biol, 10 (2014) 400-406. 
[24] S. Droby, M. Wisniewski, D. Macarisin, C. Wilson, Twenty years of postharvest biocontrol research: Is it time for a new paradigm?, Postharvest Biol Technol, 52 (2009) 137-145.

[25] R.R. Sharma, D. Singh, R. Singh, Biological control of postharvest diseases of fruits and vegetables by microbial antagonists: A review, Biol Control, 50 (2009) 205-221.

[26] Y. Elad, R. Barak, I. Chet, Y. Henis, Ultrastructural studies of the interaction between Trichoderma spp. and plant pathogenic fungi, J Phytopathol, 107 (1983) 168-175.

[27] G.E. Harman, C.R. Howell, A. Viterbo, I. Chet, M. Lorito, Trichoderma species opportunistic, avirulent plant symbionts, Nat Rev Microbiol, 2 (2004) 43.

[28] C. Wise, L. Novitsky, A. Tsopmo, T.J. Avis, Production and antimicrobial activity of 3hydroxypropionaldehyde from Bacillus subtilis strain CU12, J Chem Ecol, 38 (2012) 1521-1527. [29] E.A.B. Emmert, J. Handelsman, Biocontrol of plant disease: a (Gram - ) positive perspective, FEMS Microbiol Lett, 171 (1999) 1-9.

[30] J. Liu, I. Hagberg, L. Novitsky, H. Hadj-Moussa, T.J. Avis, Interaction of antimicrobial cyclic lipopeptides from Bacillus subtilis influences their effect on spore germination and membrane permeability in fungal plant pathogens, Fungal Biol, 118 (2014) 855-861.

[31] V. Singh, B.J. Deverall, Bacillus subtilis as a control agent against fungal pathogens of citrus fruit, Trans Br Mycol Soc, 83 (1984) 487-490.

[32] B.T. Demoz, L. Korsten, Bacillus subtilis attachment, colonization, and survival on avocado flowers and its mode of action on stem-end rot pathogens, Biol Control, 37 (2006) 68-74.

[33] L.P. Pusey, C.L. Wilson, Postharvest biological control of stone fruit brown rot by Bacillus subtilis, Plant Dis, 68 (1984) 753-756.

[34] M.V. Shternshis, A.A. Belyaev, T.V. Shpatova, A.A. Lelyak, Influence of Bacillus spp. on strawberry gray-mold causing agent and host plant resistance to disease, Contemp Probl Ecol, 8 (2015) 390-396.

[35] N.T.T. Hang, S.-O. Oh, G.H. Kim, J.-S. Hur, Y.J. Koh, Bacillus subtilis S1-0210 as a biocontrol agent against Botrytis cinerea in strawberries., Plant Pathol J, 21 (2005) 59-63.

[36] R.S. Utkhede, P.L. Sholberg, In vitro inhibition of plant pathogens by Bacillus subtilis and Enterobacter aerogenes and in vivo control of two postharvest cherry diseases, Can J Microbiol, 32 (1986) 963-967.

[37] Y.M. Jiang, X.R. Zhu, Y.B. Li, Postharvest control of litchi fruit rot by Bacillus subtilis, LWT - Food Sci Technol, 34 (2001) 430-436. 
[38] R.N. Okigbo, Biological control of postharvest fungal rot of yam (Dioscorea spp.) with Bacillus subtilis, Mycopathologia, 159 (2005) 307-314.

[39] D. Yang, B. Wang, J. Wang, Y. Chen, M. Zhou, Activity and efficacy of Bacillus subtilis strain NJ-18 against rice sheath blight and Sclerotinia stem rot of rape, Biol Control, 51 (2009) 6165.

[40] P. Shakerifard, F. Gancel, P. Jacques, C. Faille, Effect of different Bacillus subtilis lipopeptides on surface hydrophobicity and adhesion of Bacillus cereus $98 / 4$ spores to stainless steel and Teflon, Biofouling, 25 (2009) 533-541.

[41] M. Ongena, P. Jacques, Bacillus lipopeptides: versatile weapons for plant disease biocontrol, Trends Microbiol, 16 (2008) 115-125.

[42] E. Akpa, P. Jacques, B. Wathelet, M. Paquot, R. Fuchs, H. Budzikiewicz, P. Thonart, Influence of culture conditions on lipopeptide production by Bacillus subtilis, Appl. Biochem. Biotechnol, 91 (2001) 551-561.

[43] S. Buchoux, J. Lai-Kee-Him, M. Garnier, P. Tsan, F. Besson, A. Brisson, E.J. Dufourc, Surfactin-triggered small vesicle formation of negatively charged membranes: a novel membranelysis mechanism, Biophys J, 95 (2008) 3840-3849.

[44] J. Falardeau, C. Wise, L. Novitsky, T.J. Avis, Ecological and mechanistic insights into the direct and indirect antimicrobial properties of Bacillus subtilis lipopeptides on plant pathogens, J. Chem. Ecol, 39 (2013) 869-878.

[45] R. Maget-Dana, F. Peypoux, Iturins, a special class of pore-forming lipopeptides: biological and physicochemical properties, Toxicology, 87 (1994) 151-174.

[46] M. Deleu, M. Paquot, T. Nylander, Effect of fengycin, a lipopeptide produced by Bacillus subtilis, on model biomembranes, Biophys. J, 94 (2008) 2667-2679.

[47] X. Bie, Z. Lu, F. Lu, Identification of fengycin homologues from Bacillus subtilis with ESIMS/CID, J Microbiol Methods, 79 (2009) 272-278.

[48] H. Patel, C. Tscheka, K. Edwards, G. Karlsson, H. Heerklotz, All-or-none membrane permeabilization by fengycin-type lipopeptides from Bacillus subtilis QST713, BBA Biomembranes, 1808 (2011) 2000-2008.

[49] N. Vanittanakom, W. Loeffler, U. Kock, G. Jung, Fengycin-A novel antifungal lipopeptide antibiotic produced by Bacillus subtilis F-29-3, J. Antibiot, 3 (1986) 888-901. 
[50] M. Deleu, M. Paquot, T. Nylander, Fengycin interaction with lipid monolayers at the airaqueous interface-implications for the effect of fengycin on biological membranes, J. Colloid Interface Sci, 283 (2005) 358-365.

[51] Joshua N. Horn, A. Cravens, A. Grossfield, Interactions between fengycin and model bilayers quantified by coarse-grained molecular dynamics, Biophys J, 105 (2013) 1612-1623.

[52] M. Eeman, L. Pegado, Y.F. Dufrêne, M. Paquot, M. Deleu, Influence of environmental conditions on the interfacial organisation of fengycin, a bioactive lipopeptide produced by Bacillus subtilis, J Colloid Interface Sci, 329 (2009) 253-264.

[53] S. Sur, T.D. Romo, A. Grossfield, Selectivity and mechanism of fengycin, an antimicrobial lipopeptide, from molecular dynamics, J Phys Chem B, 122 (2018) 2219-2226.

[54] C. Wise, J. Falardeau, I. Hagberg, T.J. Avis, Cellular lipid composition affects sensitivity of plant pathogens to fengycin, an antifungal compound produced by Bacillus subtilis strain CU12, Phytopathology, 104 (2014) 1036-1041.

[55] S. Fiedler, H. Heerklotz, Vesicle leakage reflects the target selectivity of antimicrobial lipopeptides from Bacillus subtilis, Biophys. J, 109 (2015) 2079-2089.

[56] L.M. González-Jaramillo, F.J. Aranda, J.A. Teruel, V. Villegas-Escobar, A. Ortiz, Antimycotic activity of fengycin $\mathrm{C}$ biosurfactant and its interaction with phosphatidylcholine model membranes, Colloids Surf. B Biointerfaces, 156 (2017) 114-122.

[57] A. Roy, D. Mahata, D. Paul, S. Korpole, O. Franco, S. Mandal, Purification, biochemical characterization and self-assembled structure of a fengycin-like antifungal peptide from Bacillus thuringiensis strain SM1, Front Microbiol, 4 (2013) 332.

[58] M. Deleu, J.-M. Crowet, M.N. Nasir, L. Lins, Complementary biophysical tools to investigate lipid specificity in the interaction between bioactive molecules and the plasma membrane: a review, BBA - Biomembranes, 1838 (2014) 3171-3190.

[59] Z.V. Leonenko, A. Carnini, D.T. Cramb, Supported planar bilayer formation by vesicle fusion: the interaction of phospholipid vesicles with surfaces and the effect of gramicidin on bilayer properties using atomic force microscopy, BBA - Biomembranes, 1509 (2000) 131-147. [60] W.-C. Lin, C.D. Blanchette, T.V. Ratto, M.L. Longo, Lipid asymmetry in DLPC/DSPCsupported lipid bilayers: a combined AFM and fluorescence microscopy study, Biophys J, 90 (2006) 228-237. 
[61] S.J. Attwood, Y. Choi, Z. Leonenko, Preparation of DOPC and DPPC supported planar lipid bilayers for atomic force microscopy and atomic force spectroscopy, Int. J. Mol. Sci, 14 (2013) 3514-3539.

[62] M. Eeman, M. Deleu, From biological membranes to biomimetic model membranes, Biotechnol. Agron. Soc. Environ., 14 (2010) 719-736.

[63] L.A. Bagatolli, To see or not to see: lateral organization of biological membranes and fluorescence microscopy, BBA - Biomembranes, 1758 (2006) 1541-1556.

[64] Y.P. Patil, S. Jadhav, Novel methods for liposome preparation, Chem Phys Lipids, 177 (2014) $8-18$.

[65] E. Mantil, T. Crippin, A. Ianoul, T.J. Avis, Experimental parameters leading to optimal bilayers for total internal reflection fluorescence microscopy visualization, Microsc. Microanal, 23 (2017) 97-112.

[66] L. Gao, J. Han, H. Liu, X. Qu, Z. Lu, X. Bie, Plipastatin and surfactin coproduction by Bacillus subtilis pB2-L and their effects on microorganisms, Antonie van Leeuwenhoek, 110 (2017) 10071018.

[67] L. Zhang, C. Sun, Cyclic lipopeptides fengycins from marine bacterium Bacillus subtilis kill plant pathogenic fungus Magnaporthe grisea by inducing reactive oxygen species production and chromatin condensation, Appl. Environ. Microbiol., (2018). 


\title{
Chapter 2
}

\section{Experimental parameters leading to optimal bilayers for Total Internal Reflection Fluorescence Microscopy visualization}

\author{
E. Mantil ${ }^{1}$, T. Crippin ${ }^{2}$, A. Ianoul ${ }^{1,3}$, and T. Avis ${ }^{1,3}$ \\ ${ }^{1}$ Department of Chemistry, Carleton University, 1125 Colonel By Drive, Ottawa, ON K1S 5B6, \\ Canada \\ ${ }^{2}$ Department of Biology, Carleton University, 1125 Colonel By Drive, Ottawa, ON K1S 5B6, \\ Canada \\ ${ }^{3}$ Institute of Biochemistry, Carleton University, 1125 Colonel By Drive, Ottawa, ON K1S 5B6, \\ Canada
}

This chapter was published in the journal Microscopy and Microanalysis (2017) 23, 97-112 


\section{Abstract}

Supported lipid bilayer systems were evaluated following various experimental procedures in an effort to determine their appropriateness for visualization using total internal reflection fluorescence (TIRF) microscopy. The incorporation and distribution of Texas Red ${ }^{\circledR}$ 1,2dihexadecanoyl-sn-glycero-3-phosphoethanolamine (TR-DHPE) was studied when incorporated into bilayers of variable lipid composition using different forms of mechanical shearing. Results showed that $0.8 \%$ mol TR-DHPE provide the most optimum TIRF images. At this concentration, a sufficient level of photostability can be achieved without an undesirable increase in TR-DHPE aggregates caused by excess probe molecules. Solutions composed of a 3:1 molar ratio of DOPC:DPPC with $0.8 \%$ mol TR-DHPE produce bilayers that consistently display clear, distinct, rounded domains whereas other lipid compositions did not. This optimum phase separation appears to be influenced by an increase in mechanical shearing during the vesicle formation process, when the lipid solutions were exposed to sonication and extrusion processes. The combination of a sonication and extrusion process also helped with eliminating the presence of TR-DHPE aggregates within the model membranes. It was also shown that bilayers formed on conditioned glass, placed on a slide, produced more highly detailed bilayers in which distinct lipid phase separation could be optimally visualized using TIRF microscopy.

Key words: total internal reflection fluorescence microscopy, small unilamellar vesicles, extrusion, lipid phase separation, supported lipid bilayers 


\subsection{Introduction}

The lipid bilayer is the basis of all cellular membranes. It is vital for proper growth and survival of all cells. Cell membranes are responsible for protecting the interior contents of the cell from its external environment, while still being semi-permeable as to allow the trafficking of molecules into and out of the cell $[1,2]$. The idea of the cell membrane being a fluid mosaic model, consisting of fluid lipids with proteins (glyco- and lipoproteins) dispersed throughout, was established in 1972 [1]. In an attempt to study the biophysicochemical properties of cell membranes, Supported Lipid Bilayers (SLB) have been created and studied to help in the understanding of membranes found in nature [3, 4]. Characteristics observed in a SLB have begun to shine light on the action(s) of cell membranes.

SLB models consist of a variety of different lipids, varying in degree of saturation and overall charge, at varying molar ratios, dependent on experimental requirements. Commonly, lipid systems are composed of one lipid, which chiefly takes on a solid gel state, mixed with another lipid, which could predominantly reside- in a disordered state. For example, combinations of (but not limited to) DOPC:DPPC, DOPC:DOPE, DOPC:DOPS, DPPC:DPPE:DPPG, POPC:PG, and egg-PC:DOPE, with or without the addition of a sterol, have been used previously [5-9]. With the addition of cholesterol to these lipid solutions, an ordered state lipid phase can be introduced. These lipids have also been studied individually in SLB systems [10-13].

The vesicle fusion model using conditioned glass as the solid support is the most commonly used method [14-17]. When preparing SLB using vesicle rupture, the method used to form the Small Unilamellar Vesicles (SUV) and its impact on the final bilayer is of paramount importance. 
In particular, the methodological steps must contribute to consistent bilayer formation and bilayers that exhibit clear lipid phase separation. Being able to discern between the solid gel and disordered phases is important in different membrane studies such as the analysis of the effects of membranedisrupting compounds $[6,15,18,19]$. Clear bilayer phase separation can provide insight into the types of lipids, or other membrane components, on which these compounds act. This can help in determining a more specific mode of action for a membrane-disrupting compound of interest $[6$, $15,18,19]$.

Common protocols used in the preparation of lipid solutions to produce SUV generally involve sonication, high-pressure extrusion or a combination thereof $[8,9,12,20,21]$, which affect vesicle uniformity and/or size. The adsorption of large vesicles is thermodynamically favorable compared to the binding of small vesicles [22]. Experimentally, adsorption of vesicles as small as $25 \mathrm{~nm}$ in diameter on mica and $\mathrm{SiO}_{2}$ has been noted [23]. Larger vesicles will spontaneously rupture once adsorbed to a support although work by Dimitrievski [23] notes that an increased vesicle size does not directly lead to an increased tendency for vesicle rupture. Using sonication as a primary method for vesicle formation, SUV can be formed with a relatively low level of variance in vesicle sizing $(50 \mathrm{~nm})$ with a lower limit of approximately $10 \mathrm{~nm}$ [24]. When sonication is the only means of SUV formation, the presence of these $10 \mathrm{~nm}$ vesicles can lead to a disordered and mottled appearance due to the larger sized vesicles $(50 \mathrm{~nm})$ binding and rupturing on the support while the $10 \mathrm{~nm}$ vesicles adsorb and stay intact. The addition of the extrusion process creates a second step of shearing the already existing liposomes, forcing the formation of bilayer fragments which then close in upon themselves to produce a more energetically stable state [25]. Forcing the lipid solution created via sonication through a membrane may assist in further eliminating any multilamellar vesicles (MLV) still present in the solution. Pairing the act of mechanical shearing 
with a $50 \mathrm{~nm}$ pore size would help to influence the bilayer fragments produced and narrow the level of variance in vesicle sizes. With a higher number of large vesicles in the final solution, the number of vesicles, which become adsorbed and rupture on the support, would increase.

The slide method involves placing a conditioned coverslip over a volume of prepared liposome solution, which is then transferred to a slide [10]. The pressure created from placing the coverslip on top of the liposomes leads to a forced mechanical shearing of liposomes (Seelig \& Macdonald, 1987) - essentially rupturing the liposomes. Once open, the lipid fragments adhere to the support to create a bilayer. While domain separation is a thermodynamic property influenced by temperature and lipid type used, forced shearing has been previously shown to help orient microdomains within the bilayer [26, 27]. Forcing the opening of liposomes would reduce the likelihood of small liposomes adhering, and not rupturing on the support.

When preparing and visualizing lipid bilayers, it is also important to take into account such factors as the acyl chain length, transition temperature, and, if tagged with a fluorescent probe, the physical location of the fluorophore on the fluorescent phospholipid. Depending on the interactive forces, lipid molecules may mix evenly among each other or segregate into distinct separate domains due to the repulsive forces between one another. This repulsive force is referred to as the Gibbs free energy of interactions $(\omega)$. Gibbs free energy of interactions typically fall in the range of -300 to $+300 \mathrm{cal} / \mathrm{mol}$. In general, a higher positive $\omega$ indicates a more complete separation of lipid phases. Previous work has shown a $\omega$ equal to $+70 \mathrm{cal} / \mathrm{mol}$ when 1,2 -dipalmitoyl-sn-glycero3-phosphocholine (DPPC) and 1,2-dioleoyl-sn-3-phosphocholine (DOPC) are mixed within bilayers, indicating a distinct separation of lipid phases [28, 29]. DPPC contains saturated 16 carbon acyl chains. Due to its high level of saturation and high transition temperature (melting temperature) of $41^{\circ} \mathrm{C}$, it maintains a rigid, gel state at room temperature [30]. This is often referred 
to as the solid gel state $\left(1_{\beta}\right)$. DOPC has longer acyl chains of 18 carbons, each containing an unsaturation in the ninth position. Being unsaturated with a transition temperature of $-17^{\circ} \mathrm{C}, \mathrm{DOPC}$ remains in a fluid state (liquid disordered state; $1_{\alpha}$ ) when viewed at room temperature. Based on the phase diagram of two such lipids mixed, it is expected that a mixture of solid gel and liquid disordered states will be observed [31].

Commonly used fluorophores include DilC 18 , Texas Red, rhodamine, NBD, and BODIPY. Fluorescent probes are chosen based on their characteristics within a membrane. Some act as membrane-like compounds while others do not. Often probes are chosen based on their preference to associate within one lipid phase. Most fluorescent probes preferentially partition into the liquid disordered state $\left(1_{\alpha}\right)$ allowing identification of two separate domains [32]; the $1_{\alpha}$ containing the fluorescent probe and the dark, non-fluorescent $1_{\beta}$, which are useful for fluorescent visualization techniques. This probe characteristic would enable the visualization of the distribution of lipids within a model. Previous work indicated the use of TR-DHPE in lipid solutions at concentration between $0.1-2 \% \mathrm{~mol}[5,7,12,20,33-36]$. In some cases, up to $5 \%$ mol has been used [37]. It has been shown previously that fluorescent probes have little to no impact on the membrane dynamics when used in minute quantities $(\leq 1 \%)$ [38]. Using this as a starting point, the level of TR-DHPE was evaluated to determine an appropriate level at which an even level of fluorescence, few molecular aggregates, and limited photobleaching could be obtained when using fluorescence techniques.

Among microscopy techniques, Atomic Force Microscopy (AFM) is a powerful tool in the analysis of lipid bilayers. An AFM probe scans the surface of a bilayer providing information on the height and diameter of lipid domains. This information can be further assessed to determine the acyl-chain lengths of the lipids present. Used in rupture force experiments, AFM can provide 
details on intrinsic properties of the bilayer, such as $\mathrm{pH}$, lipid composition, ionic charge within and around the bilayer, as well as deposition pressure [39]. Another imaging tool for membrane visualization is Total Internal Reflection Fluorescence Microscopy (TIRFM). TIRFM relies on the application of an excitation light at the surface of a sample, creating an evanescent wave near the surface $(\leq 100 \mathrm{~nm})$ of the support (i.e., a glass slide, culture dishes, etc.). This evanescent wave eliminates the illumination of species in the bulk water phase of the sample of interest, ultimately producing a more clear, well detailed image [40]. The elimination of background interference gives TIRFM an advantage over epifluorescence or confocal microscopy [41-43]. TIRFM is commonly used in the imaging of cells for determination of cell morphology and cellular dynamics; be it cell signaling, surface receptors or proteins, ion channels, or substrate binding sites [40]. TIRFM can also be used to view single molecule fluorescence at the support. This is ideal when viewing SLB containing a fluorescent probe, or multiple probes, making it a useful technique in biomembrane analysis $[30,35,36,44]$.

Whereas much work has employed SLB in membrane studies using AFM, there is comparatively less work on the use of SLB for fluorescent studies including TIRFM. Fluorescence microscopy relies mainly on typical image analysis approaches. It as a useful technique when evaluating the movement/interaction of molecules with or within a membrane [44]. Fluorescence microscopy techniques also typically rely on the use of large intact unilamellar vesicles $[31,45$, 46]. In the present study, different methodological preparations of SLB from SUV were produced to determine their appropriateness for TIRFM analysis. SLB were produced via spontaneous rupture of SUV on conditioned glass supports with a large aqueous layer above the sample $(1 \mathrm{~cm})$ as well as produced via forced rupture of SUV with a minimal bulk water phase above the bilayer (approximately 100 micrometer). 
Because of the rather broad diversity of protocols use to prepare SLB for visualization using TIRF microscopy, the aim of this work was to identify experimental parameters promoting the even distribution of fluorophores within a bilayer and the evaluation of domain distribution of DOPC and DPPC. This work specifically evaluated (i) the photostability and distribution of fluorescent probes, (ii) the effect of sonication and extrusion on domain formation and distribution as well as probe distribution, and (iii) the effect of lipid composition on the formation of bilayers with consistent and clear domain separation for use in visualization experiments with TIRFM.

\subsection{Materials and Methods}

\subsubsection{Materials}

1,2-Dioleoyl-sn-glycero-3-phosphocholine （DOPC), 1,2-dipalmitoyl-sn-glycero-3phosphocholine (DPPC), and 1,2-dimyristol-sn-glycero-3-phosphoethanolamine-N-(7-nitro-21,3-benzoxadiazol-4-yl, triethylammonium salt) (NBD-PE) were purchased from Avanti Polar Lipids (Alabaster, AL, $\quad$ USA). $\quad$ Texas $\quad \operatorname{Red}{ }^{\circledR} \quad$ 1,2-dihexadecanoyl-sn-glycero-3phosphoethanolamine, triethylammonium salt (TR-DHPE) was obtained from Life Technologies (Burlington, ON, Canada). BODIPY® $\quad N$-(4,4-difluoro-5,7-dimethyl-4-bora-3a,4a-diaza-sindacene-3-propionyl)-1,2-dihexadecanoyl-sn-glycero-3-phosphoethanolamine,

triethylammonium salt (BODIPY FL-DHPE) was purchased from Thermo Fisher Scientific (Burlington, ON, Canada). Reagent grade chloroform was obtained from Caledon Laboratories (Georgetown, ON, Canada). The lipid solution buffer used in all experiments contained BioPerformance Certified ( $\geq 99.0 \%$ ) Trizma ${ }^{\circledR}$ hydrochloride (Tris-HCl; Sigma-Aldrich, Oakville, ON, Canada), calcium chloride ( $\mathrm{CaCl}_{2}$; Sigma-Aldrich), sodium chloride ( $\mathrm{NaCl}$; BioShop Canada, 
Burlington, ON, Canada), and Milli-Q water deionized to a resistivity of $18.2 \mathrm{M} \Omega \mathrm{cm}$. Glass coverslips (\#1.5) from Warner Instruments (Hamden, CT, USA) were conditioned in a boiling Sparkleen (Thermo Fisher Scientific) solution for ten min, followed by washing with copious amounts of distilled water for $20 \mathrm{~min}$. After rinsing, coverslips were dried in a $343^{\circ} \mathrm{C}$ kiln for a minimum of six hours.

\subsubsection{Small Unilamellar Vesicle (SUV) Preparation}

\subsubsection{General Vesicle Preparation Protocol}

Lipid solutions were produced as previously described [12, 20, 21, 37, 47, 48]. Briefly, lipid stock solutions were prepared, using chloroform, in glass vials. Aliquots of stock solutions were used to obtain solutions with a DOPC:DPPC molar ratio of 1:1. Initially, all lipid solutions contained $0.8 \%$ mol TR-DHPE.

Chloroform was evaporated under vacuum for a minimum of one hour or until all traces of solvent were absent. The lipid films were rehydrated with SLB buffer $\left(3 \mathrm{mM} \mathrm{CaCl}_{2}, 10 \mathrm{mM}\right.$ Tris$\mathrm{HCl}, 100 \mathrm{mM} \mathrm{NaCl} ; \mathrm{pH} 7$ ) to a final concentration of $0.75 \mathrm{mg}$ lipids $/ \mathrm{ml}$, and lipids were hydrated at $80^{\circ} \mathrm{C}$ for one hour. The hydrated lipid solution was then sonicated for one hour in a water bath $\left(80^{\circ} \mathrm{C}\right)$. After sonication, lipids were left to cool in the water bath until room temperature was obtained. Solutions were then stored at $4^{\circ} \mathrm{C}$ for no longer than 4 days, at which point vesicle deterioration became noticeable. 


\subsubsection{Effect of Lipid Probe}

Lipid solutions were prepared as described above with the exception of lipid probe used. First, lipid solutions were initially prepared with either $0.8 \%$ mol TR-DHPE, $0.8 \%$ mol BODIPY FL-DHPE or 1\% mol NBD-PE. In a second trial, TR-DHPE was evaluated at concentrations ranging from $0.1-0.8 \%$ mol. These concentrations were chosen to reflect what was found in the relevant literature. Bilayers were viewed both in chambers and on slides as described below.

\subsubsection{Effect of Sonication}

Lipid solutions were prepared as described above. The solutions were sonicated at $80^{\circ} \mathrm{C}$ for either 1 or 2 hours, using an ultrasonic cleaner. These two sonication times were performed for all lipid solutions containing 0.1-0.8\% mol TR-DHPE. Bilayers were viewed both in chambers and on slides as described below.

\subsubsection{Effect of Extrusion}

Lipid solutions were prepared as described above, with an additional extrusion process after one hour of sonication. Following sonication, solutions were manually extruded using gas tight glass syringes and polycarbonate membranes $(0.05 \mu \mathrm{m})$, according to manufacturer's instructions (Avanti Polar Lipids). Extrusion apparatus was kept at $60^{\circ} \mathrm{C}$ during the entire process. Bilayers were viewed both in chambers and on slides as described below. 


\subsubsection{Effect of Lipid Composition}

Lipid solutions were prepared as described above. Solutions were altered to contain either pure DOPC, molar ratios of 3:1 DOPC:DPPC, 1:1 DOPC:DPPC or 1:3 DOPC:DPPC, or pure DPPC. Each solution contained $0.75 \mathrm{mg}$ total lipids/ml. TR-DHPE concentration was maintained at $0.8 \%$ mol for all treatments. Half of each solution was exposed to an additional extrusion process as described in the previous section. Bilayers were viewed both in chambers and on slides as described below.

\subsubsection{Bilayer Formation}

Supported lipid bilayers (SLB) were prepared via the vesicle fusion method $[10,12,14$, $17,20]$.

\subsubsection{Preparation in Chambers}

Conditioned coverslips $(18 \mathrm{~mm})$ were placed in a Chamlide $35-\mathrm{mm}$ polycarbonate magnetic dish (Quorum Technologies, Guelph, ON, Canada), with an internal volume of approximately one millilitre, along with $75 \mu \mathrm{l}$ of vesicle solution and $150 \mu \mathrm{l}$ of $\mathrm{CaCl}_{2}(15 \mathrm{mM})$. The solution was incubated for one hour at $60^{\circ} \mathrm{C}$. After incubation, chambers were left to cool to room temperature before rinsing off excess lipids with Milli-Q water. Schematic experimental setup is shown in Fig. 1a. 


\subsubsection{Preparation on Slides}

Seventy-five $\mu \mathrm{L}$ of vesicle solution was aliquoted into the bottom of a crystallization dish and covered with a conditioned coverslip. Coverslips were incubated at $60^{\circ} \mathrm{C}$ for $10 \mathrm{~min}$. After incubation, a two-inch layer of Milli-Q water was poured over top. Working within the water layer, coverslips were lifted and shaken for 10 seconds to remove excess lipids. Double sided tape was used to anchor the edges to the slide, as previously suggested by Brian \& McConnell [10]. Slides were removed from the water and excess water on the slide was removed. Schematic experimental setup is shown in Fig. 1b.

A

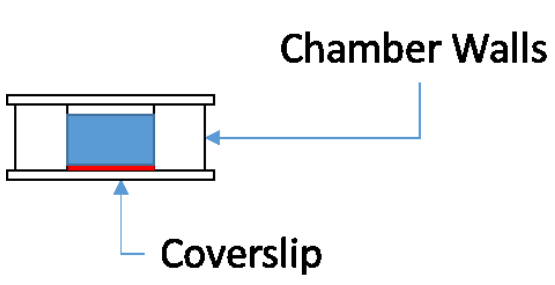

B

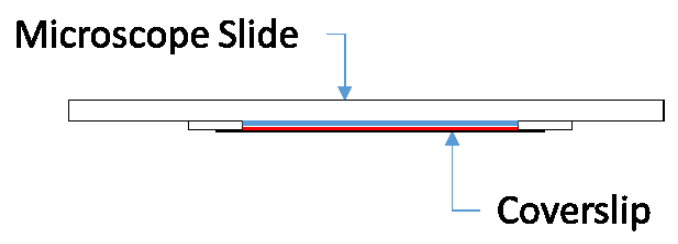

Figure 2.1. Experimental setups use for Total Internal Reflection Fluorescence Microscopy. A. Chamber apparatus containing the lipid bilayer coated coverslip. B. Microscope slide setup containing the lipid bilayer. Red $=$ phospholipid bilayer; blue $=$ aqueous layer covering the bilayer.

\subsubsection{TIRF Imaging}

TIRF images for all bilayers were carried out on an IX83 inverted microscope (Olympus Canada, Toronto, ON, Canada), equipped with a $473 \mathrm{~nm}$ diode laser, a $568 \mathrm{~nm}$ diode-pumped solid state (DPPS) laser, and a 60X TIRF oil immersion objective with a numerical aperture (NA) of 1.49. Z-Drift compensation was enabled on the IX3 microscope of the cellTIRF-4Line system (Olympus Canada). Bilayers containing BODIPY FL-DHPE were excited using the 473 and 568 $\mathrm{nm}$ lasers, separately, and emissions were collected using a multicube. Bilayers containing NBD- 
PE were excited via the $473 \mathrm{~nm}$ lasers and emissions collected using a multicube. Bilayers containing TR-DHPE were excited with the $473 \mathrm{~nm}$ and $568 \mathrm{~nm}$ lasers, separately, with emissions being collected using the multicube and the LF561 cube, respectively. Lasers used had a polarization ratio of 100:1 and were vertically oriented. Fluorescent images were collected using

a Rolera EM-C ${ }^{2}$ camera (QImaging, Surrey, BC, Canada). An electron-multiplying (EM) gain of 1 and $10 \mathrm{MHz}$ digitizer were used when acquiring images. Exposure times used for the $473 \mathrm{~nm}$ and $568 \mathrm{~nm}$ were $15 \mathrm{~ms}$ and $2 \mathrm{~ms}$, respectively. When using multiple lasers to excite the same sample region, there were no differences in the images with respect to the features visualized and therefore images from only one of the tested excitation wavelengths are provided. Fluorescence levels were thresholded and images were analyzed using MetaMorph ${ }^{\circledR}$ Advanced version 7.8.2.0 software (Molecular Devices, Sunnyvale, CA, USA). Fluorescence Recovery after Photobleaching (FRAP) was performed to ensure that images obtained were those of intact bilayers. Three trials were performed for each combination of experimental parameters.

\subsection{Results}

\subsubsection{Effect of Type of Lipid Probe}

Initial trials were completed using lipid solutions composed of 1:1 DOPC:DPPC with either 1\% mol NBD-PE, $0.8 \%$ mol BOPIDY FL-DHPE, or 0.8\% TR-DHPE. Overall, bilayers containing NBD-PE and BODIPY FL-DHPE were found to photobleach almost instantaneously (data not shown). Poor photostability of these fluorophores has been noted in the literature [4951]. Both the 473 and $568 \mathrm{~nm}$ lasers were used in the imaging of BODIPY FL-DHPE as they were the closest to its known excitation at $505 \mathrm{~nm}$. Due to rapid photobleaching, the lasers were 
attenuated to their lowest power output. However, laser attenuation did not prevent rapid photobleaching. In the few cases where images could be obtained with NBD-PE and BODIPY FLDHPE, they were of much lower quality (uneven fluorescence producing indistinct domains) than those containing TR-DHPE. Bilayers containing TR-DHPE at the tested concentration $(0.8 \% \mathrm{~mol})$ produced consistent bilayers with relatively distinct domain formation and few probe aggregates, as seen in Fig. 2.20.

\subsubsection{Effects of Lipid Probe (TR-DHPE) Concentration and Sonication}

Lipid solutions containing 1:1 DOPC:DPPC were tested with varying levels of TR-DHPE (0.1-0.8\%). Half of the lipid solutions were sonicated for an additional hour in an effort to reduce TR-DHPE clusters found in the lipid bilayers. Eliminating TR-DHPE clusters was pursued in an attempt to obtain a more homogeneous distribution of the probe to assist in clear visualization of lipid phases. When viewing the bilayers in the chamber setup (Fig. 2.2), there was very little difference between size, number, and distribution of clusters with an additional hour of sonication.

Concerning probe concentration, less definition among the bilayer was seen in the chamber setup when the lipid solution contained $0.4-0.7 \%$ mol TR-DHPE (Figs. 2.2g-n). Specifically, the separation between the liquid and solid states was less visible at these concentrations. The lower TR-DHPE levels (0.1-0.3\% mol; Figs. 2.2a-f) and the highest level tested (0.8\% mol; Figs. 2.2op) produced bilayers with clear distinctions between domains. At the lower TR-DHPE levels, 0.1$0.5 \% \mathrm{~mol}$, the lower number of total fluorescent compounds caused rapid photobleaching to occur 
when compared to higher TR-DHPE concentrations, creating difficulties in focusing and obtaining clear images of the bilayer.

When viewing bilayers using the slide setup (Fig. 2.3), more details could be generally seen when compared to images obtained using the chamber setup (Fig. 2.2). Indeed, in all cases, with
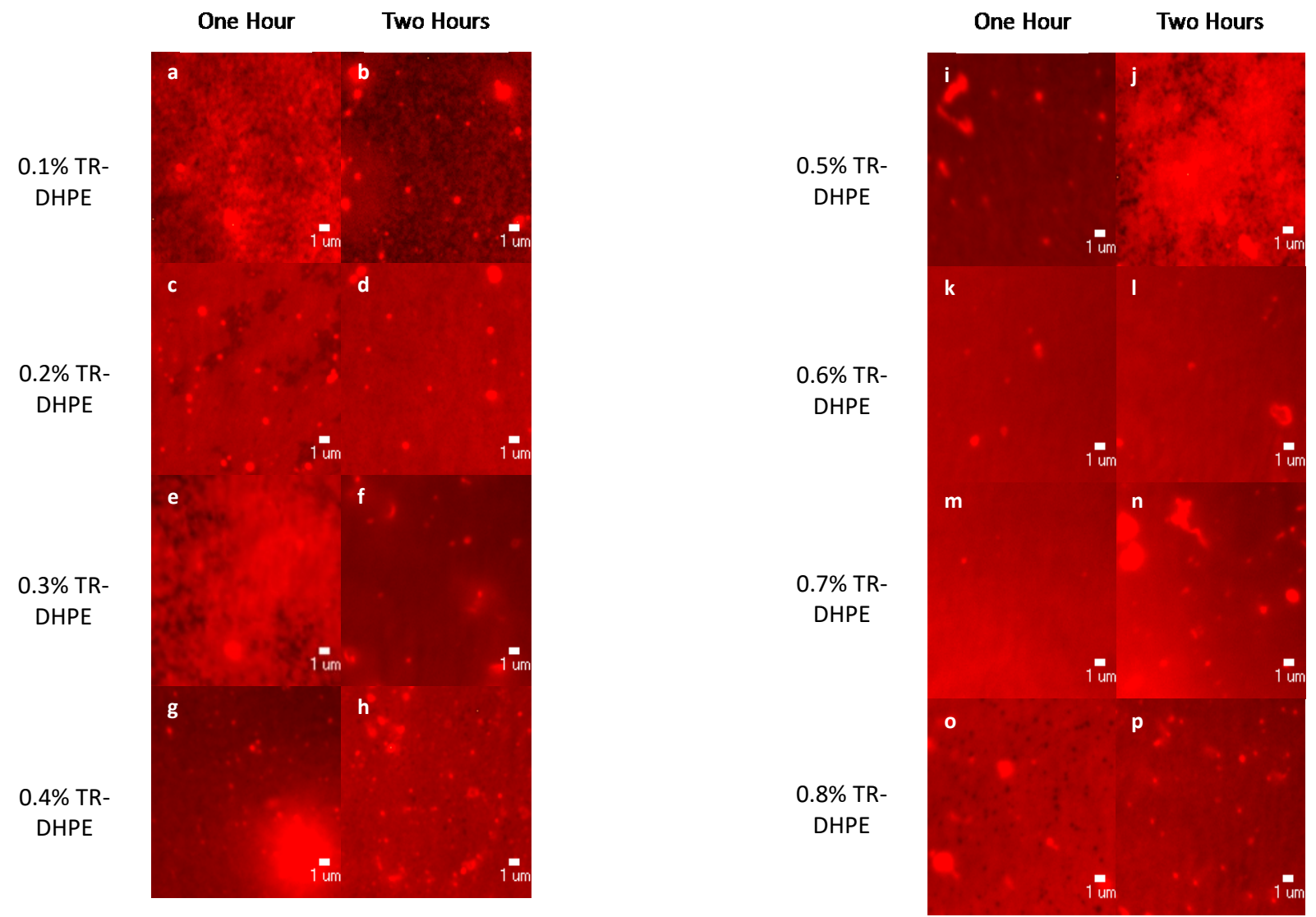

Figure 2.2. Effect of fluorescent probe concentration and sonication time on supported lipid bilayer visualization using the chamber setup. Bilayers were prepared using 1:1 DOPC:DPPC lipid solutions containing $0.1-0.8 \%$ mol TR-DHPE, as indicated. Lipid solutions were prepared using one (a, c, e, g, i, k, $\mathrm{m}, \mathrm{o})$ or two hours (b, d, f, h, j, l, n, p)of sonication. Scale bars $=1 \mu \mathrm{m}$.

the exception of $0.3 \% \mathrm{~mol}$ TR-DHPE, only small TR-DHPE aggregates could be observed. The extra hour of sonication did in some cases reduce the number of disordered domains consisting mainly of TR-DHPE, specifically those containing $0.1-0.4 \%$ mol TR-DHPE. The opposite trend was seen among solutions containing $0.5-0.8 \%$ mol TR-DHPE. 
In addition, all probe levels produced detailed images in which the solid gel and disordered domains are distinct from one another in the slide trials (Fig. 2.3). Similar to the chambers, the $0.8 \%$ mol solution was more optimal as limited photobleaching occurred. There did appear to be slightly more probe clusters in the $0.8 \%$ mol TR-DHPE solution, but when all repetitions were evaluated, it was not significantly greater than those of the lower probe levels $(\mathrm{P}>0.05)$.
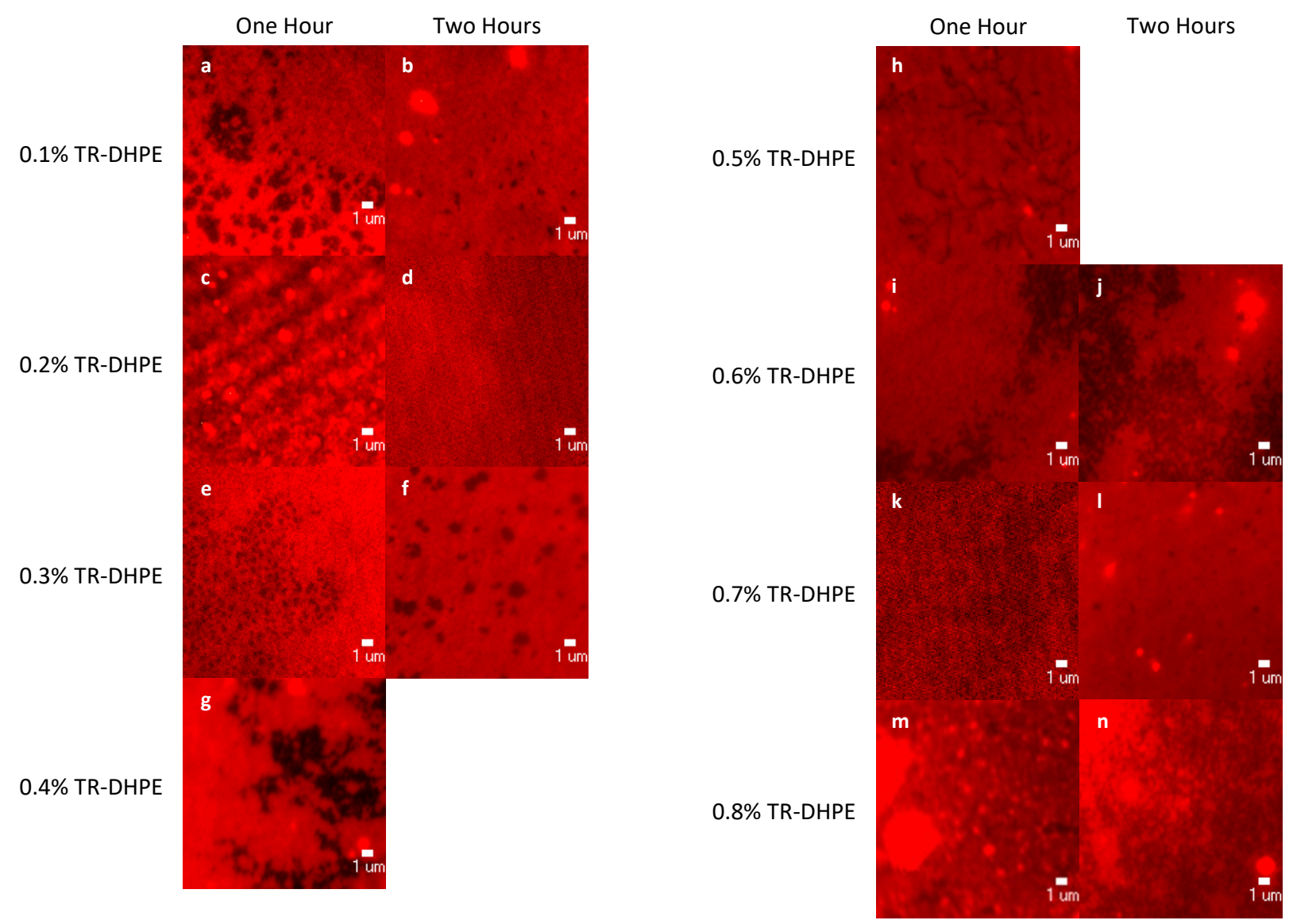

Figure 2.3. Effect of fluorescent probe concentration and sonication time on supported lipid bilayer visualization using the slide setup. Bilayers were prepared using 1:1 DOPC:DPPC lipid solutions containing $0.1-0.8 \%$ mol TR-DHPE, as indicated. Lipid solutions were prepared using one (a, c, e, g, h, i, $\mathrm{k}, \mathrm{m}$ ) or two hours (b, d, f, j, l, n). Scale bars $=1 \mu \mathrm{m}$. 


\subsubsection{Effect of Extrusion}

Similar to the previous tested trials, lipid solutions of 1:1 DOPC:DPPC with varying levels of TR-DHPE (0.1-0.8\%) were evaluated along with the addition of an extrusion process during solution preparation. When viewing bilayers using the chamber set up, the sonicated solutions created an even bilayer with a mottled appearance (Fig. 2.4). This appearance resembled an even distribution of sub-micron domains of solid gel and disordered states. Larger clusters of TR-DHPE concentrated domains $(1-10 \mu \mathrm{m})$ were present in these bilayers, along with many sub-micron clusters of the TR-DHPE probe. The solutions that went through the additional extrusion process, created similar bilayers with a few exceptions. Small aggregates of TR-DHPE $(\leq 1 \mu \mathrm{m})$ were still present, but fewer of the larger aggregates could be seen. These larger aggregates were overall smaller $(1-5 \mu \mathrm{m})$ than those seen in the solution that was only sonicated (Fig. 2.4). 


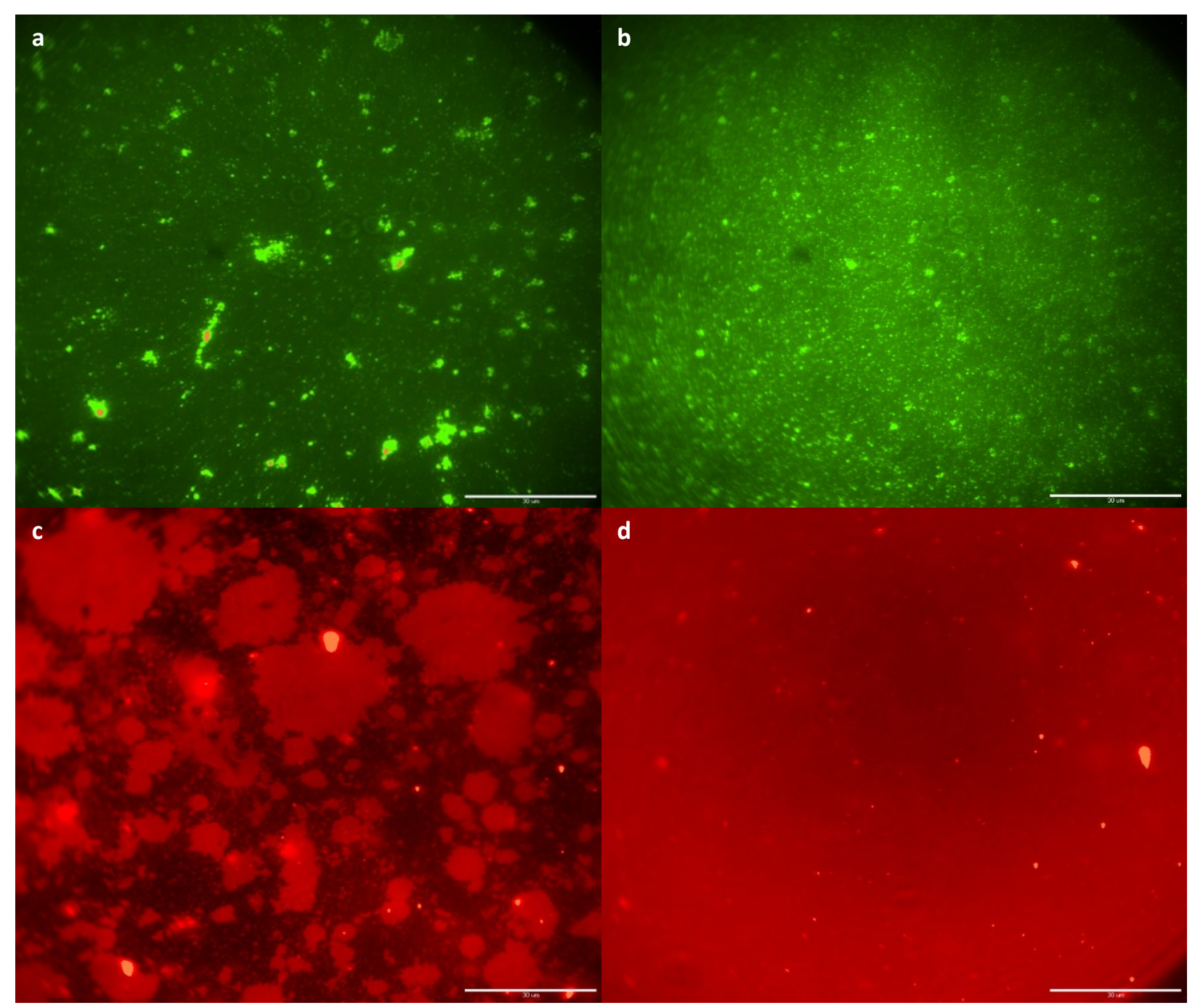

Figure 2.4. Effect of extrusion on supported lipid bilayer visualization. Bilayers were prepared using 1:1 DOPC:DPPC lipid solutions containing $0.8 \%$ mol TR-DHPE. Bilayers were visualized using the chamber $(a, b)$ and slide $(c, d)$ setup. Lipid solutions were prepared using sonication $(a, c)$ or sonication with an additional extrusion process $(\mathrm{b}, \mathrm{d})$. Scale bars $=30 \mu \mathrm{m}$. Green images $(\mathrm{a}, \mathrm{b})$ visualized with a $473 \mathrm{~nm}$ laser and a multicube; red images (c, d) visualized with a $568 \mathrm{~nm}$ laser and a $561 \mathrm{~nm}$ cube.

When viewing bilayers on slides, the sonicated solution contains solid gel domains $(10-30 \mu \mathrm{m})$ with many smaller disordered domains $(\leq 1-3 \mu \mathrm{m})$ within them and large, expansive domains of the disordered state (DOPC and TR-DHPE) $(5-40 \mu \mathrm{m})$. Aggregates of TR-DHPE $(\leq 1-3 \mu \mathrm{m})$ could be observed within the disordered state domains. Pure DPPC domains have been recorded to be $300-700 \mathrm{~nm}$ in diameter, smaller than the values obtained here [20]. This can be attributed to the presence of smaller DOPC + TR-DHPE domains within the DPPC domains. The extruded solution 
did not exhibit distinct DPPC domains. An even base fluorescence was observed indicating a thorough mixing of DPPC into DOPC. The separate domains were so small that they appeared to be evenly mixed and visual separation could not be observed. Small concentrations of TR-DHPE $(\leq 1-3 \mu \mathrm{m})$ may still be in this solution indicating that DPPC may be displacing the TR-DHPE and allowing it to aggregate with itself.

\subsubsection{Effect of Lipid Composition}

In addition to general lipid solution containing a molar ratio of 1:1 DOPC:DPPC (Fig. 2.4), lipid solutions with molar DOPC:DPPC ratios of 1:0, 3:1, 1:3, and 0:1 were viewed and the trends in domain formation were evaluated (Figs. 2.5-8). As with the 1:1 DOPC:DPPC bilayers, half of each lipid solution was exposed to the extra extrusion process and bilayers were viewed using the chamber and slide setups.

When viewing bilayers in the chamber, the sonicated solutions of pure DOPC with $0.8 \% \mathrm{~mol}$ TR-DHPE (Figs. 2.5a and c) produced bilayers which exhibited an even level of fluorescence with randomly distributed clusters with a higher level of fluorescence (brighter). This indicated an even distribution of TR-DHPE among DOPC with aggregates of pure TR-DHPE $(\leq 1-3 \mu \mathrm{m})$. In the bilayers produced using extruded solutions (Figs. $2.5 \mathrm{~b}$ and d), a higher number of the TR-DHPE aggregates were observed. However, these aggregates were of a smaller size $(\leq 1 \mu \mathrm{m})$, and are more evenly distributed when compared to the non-extruded bilayers.

In this same experiment, viewing slides with the sonicated pure DOPC $(+0.8 \%$ mol TR-DHPE) solution (Fig. 2.5) also revealed an uneven distribution of TR-DHPE within the liquid disordered phase. This distribution showed areas of (i) even mixing of DOPC and TR-DHPE producing an even layer of fluorescence, (ii) pure DOPC with traces of TR-DHPE producing pockets ( $\leq 30 \mu \mathrm{m}$ ) 
of low fluorescence with specks of TR-DHPE, and (iii) small aggregates $(\leq 1-3 \mu \mathrm{m})$ of TR-DHPE above the threshold distribution of fluorescence (represented by a bright orange cluster). The extruded solution created a bilayer in which these separations were less apparent. They exhibited an even distribution of very small pure DOPC (black) and DOPC + TR-DHPE (red) domains. This gave an overall appearance of even fluorescence. Small aggregates of TR-DHPE could still be seen, but were smaller than those observed in the non-extruded solutions.

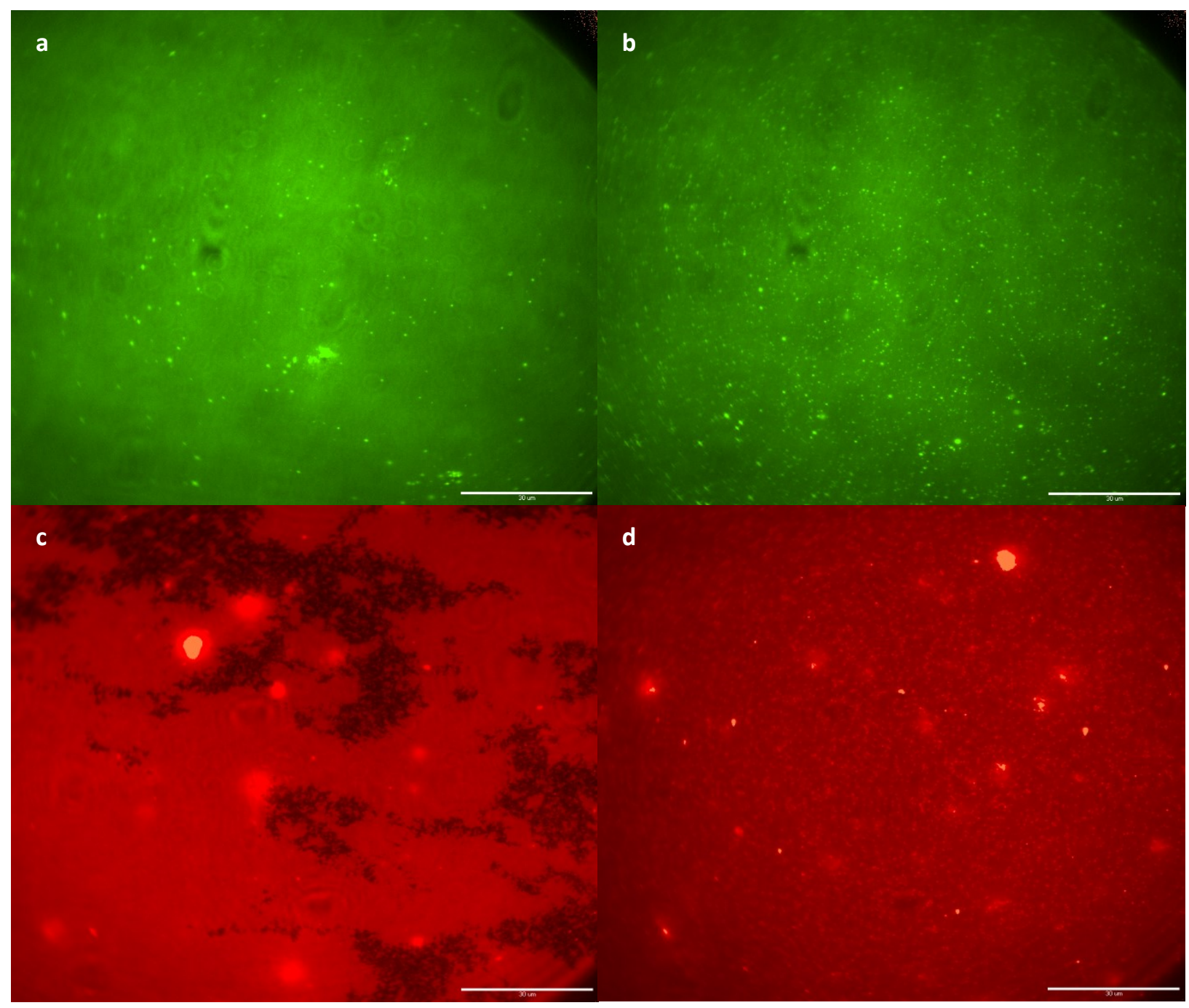

Figure 2.5. Effect of pure DOPC lipid composition on supported lipid bilayer visualization. Bilayers were prepared using $0.8 \%$ mol TR-DHPE. Bilayers were visualized using the chamber $(a, b)$ and slide $(c, d)$ setup. Lipid solutions were prepared using sonication $(a, c)$ or sonication with an additional extrusion process (b, d). Scale bars $=30 \mu \mathrm{m}$. Green images (a, b) visualized with a $473 \mathrm{~nm}$ laser and a multicube; red images $(\mathrm{c}, \mathrm{d})$ visualized with a $568 \mathrm{~nm}$ laser and a $561 \mathrm{~nm}$ cube. 
Sonicated solutions containing 3:1 DOPC:DPPC ( $+0.8 \%$ mol TR-DHPE) viewed using chambers (Figs. 2.6a and b) exhibited very small solid gel domains $(\leq 1 \mu \mathrm{m})$ among larger disordered domains $(5-15 \mu \mathrm{m})$. The small solid gel domains were mixed within slightly larger disordered domains. Small clusters with higher concentrations of TR-DHPE could also be observed. A larger number of these TR-DHPE clusters could be observed at this molar ratio when compared to pure DOPC. This indicated that in the presence of DPPC, TR-DHPE was segregated from the solid gel domains and was located within DOPC domains. The extruded solutions (Figs. 2.6b and d) did not exhibit the distinct DPPC domains observed in the solutions that had not received extrusion. An even base fluorescence were produced indicating a thorough mixing of DOPC and DPPC. The separate domains were so small that they appeared to be evenly mixed and a physical separation could not be observed. Small concentrations of TR-DHPE $(\leq 1-3 \mu \mathrm{m})$ could still be seen indicating some DPPC was displacing the TR-DHPE and forced it to aggregate with itself. 
Figure 2.6. Effect of $3: 1$ DOPC:DPPC lipid composition on supported lipid bilayer visualization. Bilayers were prepared using $0.8 \%$ mol TR-DHPE. Bilayers were visualized using the chamber $(a, b)$ and slide (c, d) setup. Lipid solutions were prepared using sonication $(a, c)$ or sonication with an additional extrusion process $(\mathrm{b}, \mathrm{d})$. Scale bars $=30 \mu \mathrm{m}$. Images visualized with a $568 \mathrm{~nm}$ laser and a $561 \mathrm{~nm}$ cube.

Viewing of the sonicated 3:1 DOPC:DPPC solutions on slides produced solid gel domains (10$30 \mu \mathrm{m})$ with many smaller disordered domains $(\leq 1-3 \mu \mathrm{m})$ within them. Large expansive domains of the disordered state $(>30 \mu \mathrm{m})$ were seen, as to be expected when the ratio of DOPC is larger than that of DPPC. Few small clusters of TR-DHPE were observed, but only within the disordered state domains. The extruded solution produced a different picture. Unlike the non-extruded solution in which the DPPC domains were amorphous with small disordered domains within, the 
solid gel domains were small and spherical. These domains lacked DOPC. These DPPC domains $(1-2 \mu \mathrm{m})$ were evenly distributed throughout the field of view, with DOPC accounting for the majority of the field of view. These bilayers were absent of TR-DHPE aggregates.

As seen in Fig. 2.7, both the sonicated (Fig. 2.7a) and extruded (Fig. 2.7b) 1:3 DOPC:DPPC solutions viewed in chambers produced an even level of fluorescence with many submicron clusters of TR-DHPE. The non-extruded lipid solutions contained a small number of larger TRDHPE aggregates (1-5 $\mu \mathrm{m})$. Many of the clusters exhibited a level of fluorescence above that of the external threshold indicating that they were not clusters of TR-DHPE, but possibly TR-DHPErich liposomes attached to the top of the bilayer. When examining the slides (Figs. 2.7c and d), large solid gel domains of pure DPPC $(8-20 \mu \mathrm{m})$ with submicron liquid disordered domains within were observed. Large solid gel domains of DPPC plus TR-DHPE $(\leq 5-20 \mu \mathrm{m})$ with virtually no clusters of TR-DHPE could also be observed. The extruded lipid solution on slides (Fig. 2.7d) produced similar results to those seen in chambers (Fig. 2.7b). 


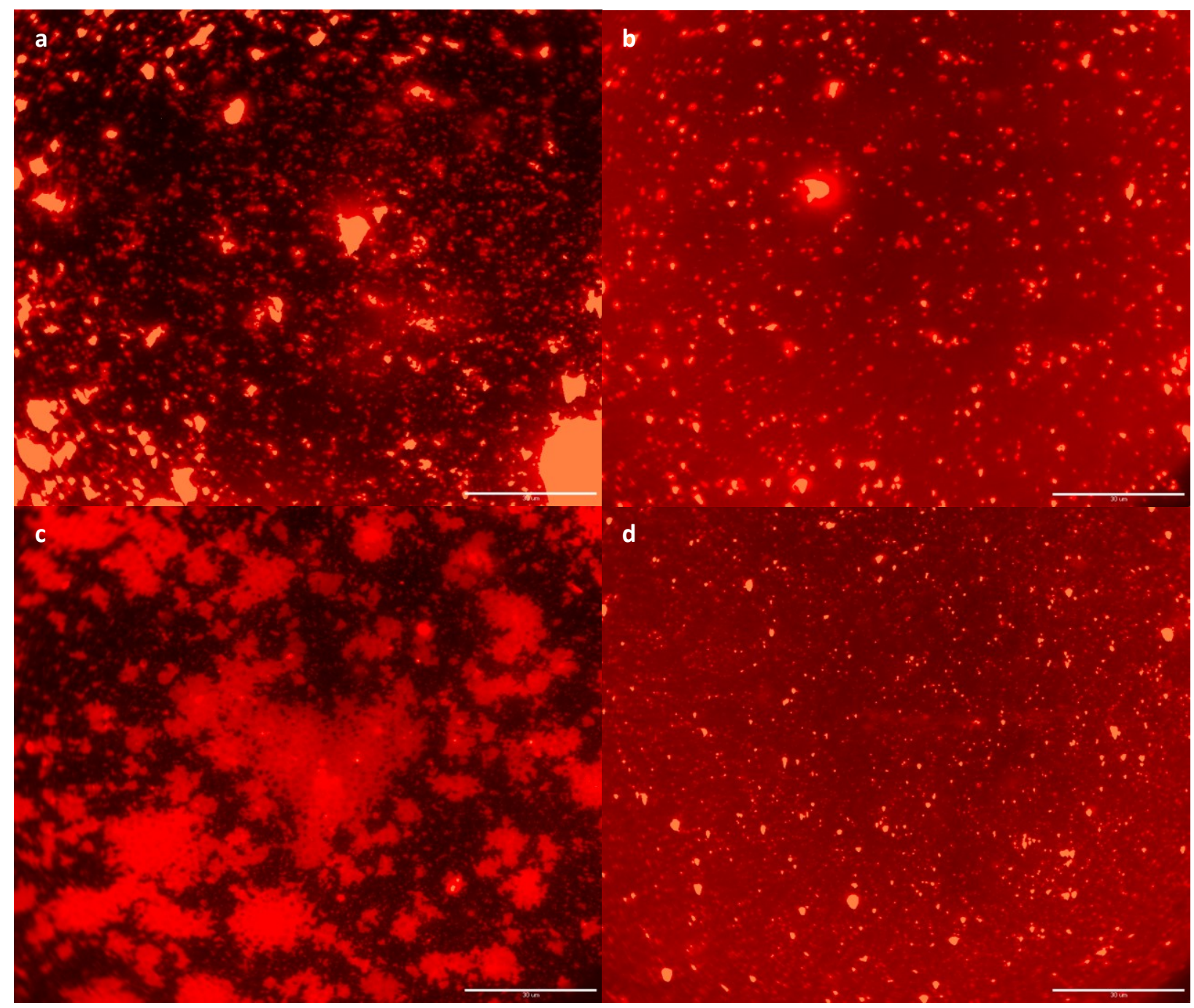

Figure 2.7. Effect of 1:3 DOPC:DPPC lipid composition on supported lipid bilayer visualization. Bilayers were prepared using $0.8 \%$ mol TR-DHPE. Bilayers were visualized using the chamber $(\mathrm{a}, \mathrm{b})$ and slide (c, d) setup. Lipid solutions were prepared using sonication (a, c) or sonication with an additional extrusion process $(\mathrm{b}, \mathrm{d})$. Scale bars $=30 \mu \mathrm{m}$. Images visualized with a $568 \mathrm{~nm}$ laser and a $561 \mathrm{~nm}$ cube.

Evaluating the pure DPPC solution using chambers, the sonicated lipid solution created a bilayer with an even distribution of fluorescence (Fig. 2.8a). Again, aggregates of TR-DHPE $(\leq 1$ $3 \mu \mathrm{m}$ ) can be observed. The extruded solution (Fig. 8b) produced similar results with the presence of small DPPC domains. These domains were difficult to observe due to the mottled appearance of the bilayer. A greater number of TR-DHPE aggregates (1-5 $\mu \mathrm{m})$ could be seen possibly because TR-DHPE will mix poorly with DPPC. This results in domains of pure TR-DHPE or TR-DHPE with a very small amount of DPPC. In this environment, TR-DHPE may be forced out of the 
bilayer due to unfavorable mixing. When observing slides, both the sonicated (Fig. 2.8c) and extruded solutions (Fig. 2.8d) produced similar results of large ordered domains of pure DPPC (8$20 \mu \mathrm{m})$ and DPPC plus TR-DHPE $(\geq 20 \mu \mathrm{m})$ with a large number of pure TR-DHPE aggregates (1-5 $\mu \mathrm{m})$. The extruded solution created a considerably lower number of the TR-DHPE clusters, which were also of a much smaller size $(\leq 1-2 \mu \mathrm{m})$. This was similar to the results observed in the extruded sample, when viewed in chambers (Fig. 2.8b), on a more exaggerated scale.

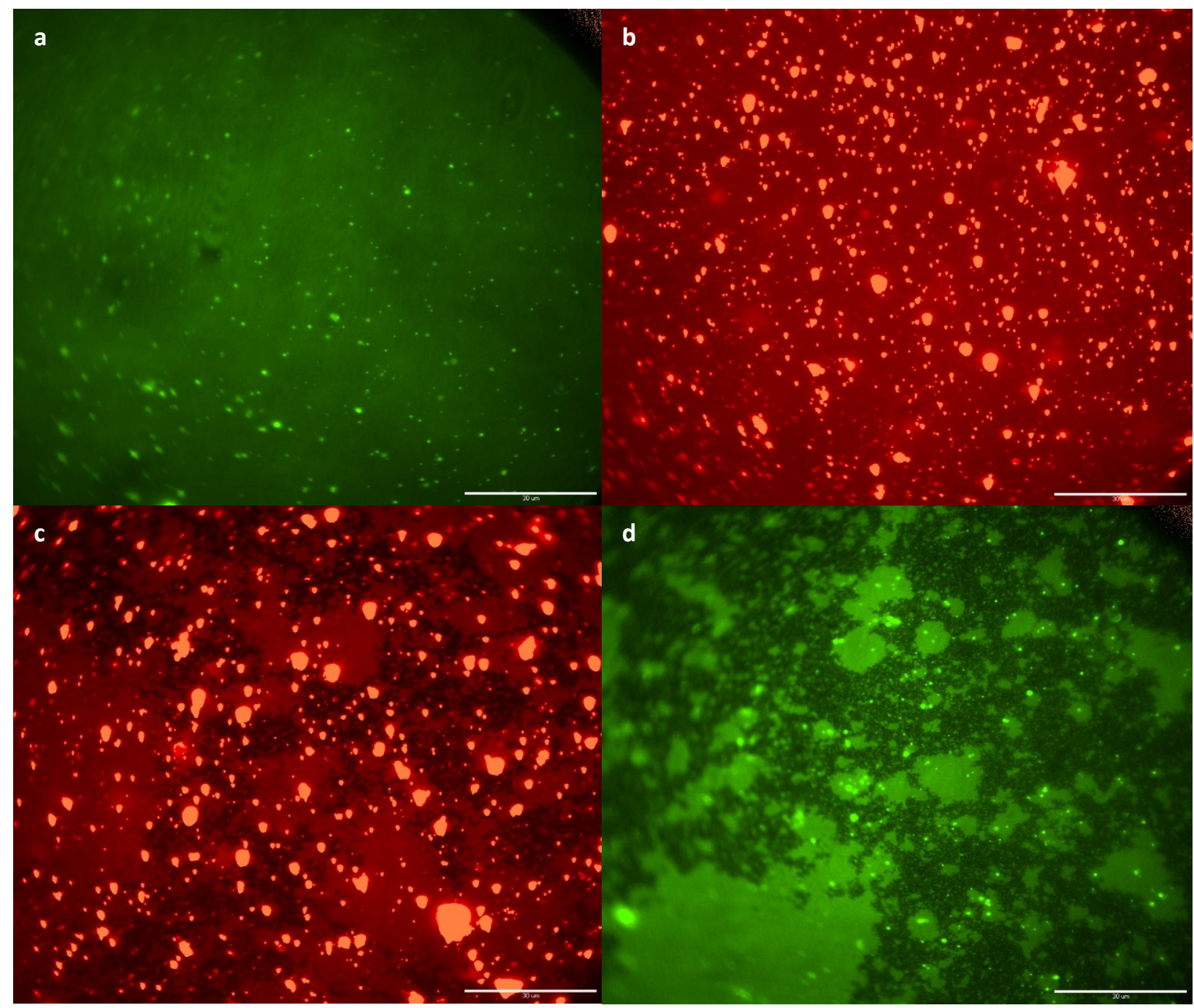

Figure 2.8. Effect of pure DPPC lipid composition on supported lipid bilayer visualization. Bilayers were prepared using $0.8 \%$ mol TR-DHPE. Bilayers were visualized using the chamber $(a, b)$ and slide $(c, d)$ setup. Lipid solutions were prepared using sonication $(a, c)$ or sonication with an additional extrusion process (b, d). Scale bars $=30 \mu \mathrm{m}$. Green images (a, d) visualized with a $473 \mathrm{~nm}$ laser and a multicube; red images (b. c) visualized with a $568 \mathrm{~nm}$ laser and a $561 \mathrm{~nm}$ cube. 


\subsection{Discussion}

TIRF microscopy is an increasingly prevalent and valuable technique used to study biomembranes. This technique is particularly useful when studying surface bound molecules, such as macromolecules, within an intact cell membrane or those found within a constructed SLB [41, 42, 52]. Previously studied SLB commonly use AFM. AFM's ability to differentiate between lipid domains relies on its ability to measure a difference in height between the two domains, and therefore the use of fluorescent probes is not required. One advantage of using TIRFM is it enables the visualization of molecular movement, whether this be in an intact cell or model membrane $[30$, 44]. Aside from experimental temperature [53,54], relatively limited information is available on the influence of methodological parameters on the distribution of a fluorescent probe in a model membrane. In this study, TIRF microscopy was used to investigate lipid bilayer characteristics when composed of varying levels of DOPC, DPPC, and TR-DHPE. In addition to changing lipid composition, the use of sonication and extrusion was performed and SLB were visualized using chamber and slide setups. These experimental processes were used to evaluate the detail in which solid gel and disordered domains were produced as well as their effects on aggregation of the fluorescent probe.

Repeated trials using NBD-PE and BODIPY FL-DHPE were not successful due to rapid photobleaching of these probes under our experimental conditions. These probes were therefore not used in additional experiments. Conversely, the fluorescent probe TR-DHPE successfully labeled the disordered domains within the bilayer without rapid photobleaching (Figs. 2.2 and 3). These results support previous work showing TR-DHPE preferentially inserting itself within the liquid disordered domains (DOPC) $[35,36,55,56]$. 
Throughout this study, a common hurdle was encountered: the TR-DHPE molecules appear to frequently aggregate with one another rather than distribute evenly throughout the lipid bilayer. These aggregates could be due to TR-DHPE within each leaflet of the bilayer interacting with each other [57] or trapping of TR-DHPE within the solid gel phase lipids (DPPC), where it has less mobility [36]. Less aggregation occurred among samples prepared using an extruder (image $d$ in Figs. 2.4-8). As previously shown, fluorescence of Texas Red is affected when forced through nano gaps [58], such as the extruder membranes, which may have reduced the presence of aggregates in the bilayer.

Results showed that chambers holding membranes containing $0.1-0.3 \%$ mol (Figs. 2.2a, c, and e) and $0.8 \%$ mol TR-DHPE (Fig. 2.20) produced detailed images with distinct contrast between $l_{\alpha}$ and $l_{\beta}$ domains. In addition, membranes containing $0.8 \%$ mol provided more photostability when compared to lower TR-DHPE concentrations - an important characteristic when evaluating membranes using fluorescence microscopy techniques. Our results showing $0.8 \%$ mol was the most optimal probe concentration used is most similar to the concentration used by Zhao et al. (2007). Higher concentrations of TR-DHPE were not tested in this study, as $0.8 \%$ was the minimal concentration used that provided appropriate visualization. TR-DHPE concentrations higher than $0.8 \%$ could not only cause excessive fluorescence, but may also begin to affect membrane dynamics if over $1 \%$ as previously described (Bouvrais et al., 2010).

Fig. $2.2(b, d, f, h, j, 1, n$, and p) showed that an extra hour of sonication did not lead to more distinct separation between domains. It is believed that the extended sonication is leading to the distribution of lipids in a manner such that the domains formed were significantly smaller. This produced a bilayer with closely spaced, alternating microdomains, producing an even level of 
fluorescence throughout and a mottled appearance. Bilayers on slides exhibited a more distinct separation between lipid domains than those formed in chambers.

It is interesting to note that, in multiple experiments, bilayers containing $0.8 \% \mathrm{~mol}$ TR-DHPE (Fig. $2.2 p$ ) produced bilayers similar to that of those containing $0.1-0.3 \%$ mol TR-DHPE (Figs. 2.2b, d, and f). Bilayers containing 0.4-0.7\% mol (Figs. $2.2 \mathrm{~h}, \mathrm{j}, 1$, and n) produced bilayers in which separate solid gel and disordered domains could not be observed when viewing the bilayers in chambers. When viewing bilayers on slides, bilayers containing 0.4 and $0.5 \%$ mol were nonreproducible (Fig. 2.3). More detail can be observed among bilayers that included an extrusion process in their protocol. TR-DHPE contains a relatively large dye moiety, making it fairly bulky. Previous work has shown that when Texas Red is forced through a nano gap, there is a lower level of fluorescence on the other side of the gap $[58,59]$. More specifically, the large dye moiety appears to inhibit the molecule's ability to pass through small channels. This results in a buildup of the molecules on the starting side of the channel $[58,59]$. This is believed to be happening during the extrusion process. At levels of $0.1-0.3 \%$ mol (Figs. $2.3 \mathrm{~b}, \mathrm{~d}$, and f), there were few enough molecules that the Texas Red can more easily pass through the pores in the polycarbonate membrane, with few being trapped before or within the membrane. When solutions contain 0.4$0.7 \%$ mol TR-DHPE (Figs. $2.3 \mathrm{j}$ and 1 ), there were still few enough molecules that they could easily pass through the membrane pores. Because almost all of the fluorescent molecules would cross the extruder membrane, the fluorescent intensity increased, as is to be expected when increasing the probe concentration. This level of fluorescence makes it more difficult to visualize distinct separations between domains. As probe concentration increases, it is to be expected that more of the probe will become trapped in the extruder membrane. At a concentration of $0.8 \%$ mol (Fig. 2.3n), larger decreases in overall fluorescence appear and more clear distinction of domain 
separations may be observed. As previously shown by Kashimura et al. [58], a decrease in fluorescent intensity of approximately $30 \%$ can be seen when the fluorescent probe is passed through a nano gap of $40 \mathrm{~nm}$. The intensity continues to decrease as the gap size is reduced. With a pore size of $50 \mathrm{~nm}$ in the polycarbonate filters used in this study, it can be suggested that significant traces of the TR-DHPE become trapped within the extruder membrane, resulting in lowering of the overall fluorescence intensity in the SLB. This may explain the decrease in fluorescent intensity seen at $0.8 \%$ mol TR-DHPE (Fig. 2.3n). This could be observed after the completion of the extrusion process, when colored traces could be seen in the membrane filters. The accumulation of colored compounds in the filter was more intense as the concentration within the solution increased. Therefore, the use of $0.8 \%$ mol TR-DHPE in combination with extrusion was the most optimal process used in this study.

In addition to the above-mentioned parameters, work was also performed on the role of lipid composition of final bilayer visualization in this system. In general, these results showed that the extrusion process led to a significant decrease, and in some cases absence of, TR-DHPE aggregates. Furthermore, bilayers on slides produced more detailed results than in chambers as in other trials in this study. Extruded solutions are thought to contain a more even distribution of lipids within the vesicles. Moreover, distinct separation among TR-DHPE could be observed. This trend was also observed on bilayers formed on slides (Figs. 2.3-2.7), whereby clearer distinction between TR-DHPE rich domains ( $l_{\alpha}$ domains) and those without ( $l_{\beta}$ domains) could be observed.

As previously stated, extrusion can help to decrease the variance in vesicle size, producing a higher number of uniform vesicles which improves the adsorption and rupturing of vesicles on the planar support. This can be observed in the improvement of bilayer formation seen in the figures produced using the extrusion process. Production and redistribution of bilayer fragments would 
also introduce the potential for a more thorough mixing of lipids within vesicles. This may result in a more even distribution of domains (Fig. 2.6d). The increase in lipid mixing and decrease in vesicle size variances become more pronounced when bilayers were prepared using the slide method. Forced mechanical shearing of the liposomes helps to improve orientation of microdomains [26, 27]. This is visible in all of the figures provided. The degree of clarity and details visible is therefore greatly increased in bilayers using the slide setup. As mentioned previously, by including an extrusion step prior to transferring to the slide setup, better mixing of the lipids would be achieved and, unlike the bilayers formed in chambers, those transferred to slides show this greater clarity and detail in the experiments performed.

When lipid composition was specifically evaluated, many trends in domain formation were found. An equal ratio of lipids (1:1 DOPC:DPPC) led to distinct separation of the solid gel and disordered domains, but when extruded, these distinct separations are harder to see. This may be due to better distribution of lipids within SUV resulting in even smaller domains with random mixing. Pure DOPC solutions created an even layer of fluorescence with many pockets rich in TRDHPE. When these pure DOPC solutions were only sonicated, areas in which TR-DHPE did not partition could be observed. This further supported the use of extrusion to assist with providing a more even distribution of lipids.

When studying the bilayers on slides, those which were only exposed to a sonication period produced fractal domains (image $\mathrm{c}$ in Figs. 2.4-2.8). There existed larger disordered domains with amorphous, feathery edges. Within the large $l_{\alpha}$ domains, $l_{\beta}$ of only a couple of microns in size were observed. The opposite trend was revealed within the large solid gel domains. This pattern of domains suggested an unstable growth of domain phases [16]. Along with only the use of 
sonication, the use of conditioned glass has been shown to support the production of fractal domains [16].

As seen in Figs. 2.3, 2.4, and 2.6, fractal domains were formed after the sonication period (Figs. 2.3a, c, e, g, h, I, k, and m; Fig. 2.4c; Fig. 2.6c), but with the addition of an extrusion process (Figs. 2.3b, d, f, j, 1, and n; Fig. 2.4d; Fig 2.6d), the fractal domains were replaced by many submicron domains producing a mottled appearance. This trend has previously been noted when bilayers are heated close to their $T_{m}[16]$. The bilayers shown in Fig. 2.3 and Fig. 2.6d were imaged at room temperature, showing that the extrusion process may produce similar effects to that of increasing the temperature of the environment. When lipids of both phases are brought close to their $T_{m}$, the lipids become more mobile. This increases the lateral mobility of lipids, creating more evenly mixed lipids and, as a result, a more homogeneous distribution of the fluorescent probe. This created bilayers with virtually no distinct domains, referred to as a Super Lattice model $[16,60]$. Fig. 2.4 shows the bilayer of a solution containing a 1:1 ratio of DOPC:DPPC in which the application of an extrusion process appeared to be forcing a more even mixing of the lipids within the SUV produced. It was not surprising to see the same mottled appearance in Fig. 2.5, as the lipid solution is composed of pure DOPC and TR-DHPE. Because TR-DHPE preferentially partitions into DOPC, an even level of fluorescence throughout the bilayer was expected. For pure DOPC bilayers, domains absence of fluorescence would only be expected when a low concentration of TR-DHPE is used, which was not the case with $0.8 \%$ mol of the probe. Conversely, it is somewhat surprising to see an even layer of fluorescence in bilayers comprised of pure DPPC (Fig. 2.8). TR-DHPE had been shown to partition into DPPC only if in the presence of a DPPC-rich environment $[34,57,61]$. This was supported by the large number of highly fluorescent domains, clearly composed of pure TR-DHPE (Figs. 2.8a and b). The even level of 
fluorescence obtained in these bilayers indicated that the use of an extrusion process helps to more evenly distribute lipids within SUV. The motion of forcing lipids through a pore mechanically shears liposomes producing lipid bilayer fragments [25]. This additional process of shearing and creating bilayer fragments may create an optimal environment for further mixing and lipid rearrangement before the fragments once again fold into liposomes. Extrusion may therefore promote a more even distribution of lipids among SUV and even domain formation throughout the SLB.

As shown in Figs. 2.6 and 2.7, altering the lipid molar ratios to 1:3 and 3:1 DOPC:DPPC allows distinct domain formation in the sonicated (Figs. 2.6c and 2.7c) and in the sonicated and extruded solutions (Figs. 2.6d and 2.7d) deposited on slides. More specifically, Fig. 2.7 showed the potential difference in distribution of lipids within differently prepared SUV. When the 1:3 DOPC:DPPC bilayer was exposed to only the sonication process (Fig. 2.7c), large fractal domains were formed. Within almost all of the fractal disordered domains, there were submicron solid gel domains with rounded edges. This greater coverage of solid gel domains would be expected when the ratio of DPPC is higher than that of DOPC. Indeed, the repulsion experienced by the two lipids, domains will still form, but the smaller quantity of DPPC within the membrane will produce separate domains smaller than that of DOPC. Conversely, as seen in Fig. 2.6d, the field of view consisted primarily of the disordered phase because it was composed primarily of DOPC, which maintained a liquid disordered phase when at room temperature.

Of all the lipid compositions, achieving consistent bilayer formation was most difficult when using the 1:3 DOPC:DPPC lipid solution. This may be due to the difficulty of TR-DHPE mixing with DPPC, creating an unfavorable environment in which the exclusion force is too large [28, 29]. Strong repulsive forces will cause lipids to separate and form two distinct domains [28, 31]. 
In this case, the large number of DPPC molecules would cause expulsion of the TR-DHPE out of the bilayer unless it was able to organize with the DOPC. With limited DOPC in this model, this became challenging, leading to difficulties in visualizing this membrane without sufficient presence of the probe. With the addition of an extrusion step, distinction between domains is lost in the 1:3 DOPC:DPPC solution. Clustering of TR-DHPE was also reintroduced after this step, similar to the bilayers composed of pure DPPC. Again, this was likely due to TR-DHPE's preference to not partition with DPPC. Therefore, these factors make the 1:3 DOPC:DPPC a nonideal model bilayer to study using this system.

The solution containing a 3:1 DOPC:DPPC ratio produced clear distinct, rounded domains in our system of study (Fig. 2.7d). These domains are highly sought after and generally considered the ideal domain shape $[5,20,48,62]$. The mechanical shearing of SUV in the extrusion process appeared to influence the separation of like lipids. Separating the lipids would increase the probability of them interacting with other components within the solution, resulting in a better mixing of the lipids, thus achieving even size and distribution of DPPC domains within the bilayer with this lipid composition.

Overall, a lipid composition of 3:1 DOPC:DPPC with $0.8 \%$ mol TR-DHPE (Fig. 2.7d) provided the best visualization of SLB using TIRFM in our system of study. When processing the lipid solution, sonication with an additional extrusion process yielded a bilayer virtually absent of TR-DHPE aggregates. While these parameters have been most optimal for viewing SLB in this work as well as SLB containing variable amounts of sterols and SLB prepared from complex lipid extracts (unpublished data), these parameters may not be optimal in all cases.

TIRFM is an excellent tool when qualitatively evaluating lipid bilayers. As seen in previous work, TIRFM can be used to evaluate substrate binding of a cell membrane $[40,52,63]$. This could 
be evaluated further by looking more closely solely at the phospholipid bilayer when in contact with single molecules, such as known membrane disruptors with unknown modes of action. Association of the compound and bilayer can be seen via TIRFM before it may be detectable with AFM [20]. By evaluating such interactions, it becomes possible elucidate the precise mode of action and determine factors that affect membrane-targeting compound activities (e.g., overall lipid charge, acyl chain lengths, membrane fluidity, etc.). TIRFM is, however, limited, in that it cannot be used to take topographical quantitative measurements. Such measurements are useful, for example, in the determination of acyl chain tilt, giving insight into how such qualities affect separations of lipid phases in ternary systems [16]. Therefore, TIRFM can be a valuable addition to AFM and other techniques in gaining additional information on interactions occurring in biomembranes.

\subsection{Conclusions}

Neutral bilayers were formed via vesicle fusion on conditioned glass. Using TIRF microscopy, the domain formation seen in a mixture composed of DOPC, DPPC, and TR-DHPE was evaluated. Specifically, TIRF microscopy was used to evaluate the distribution of TR-DHPE in bilayers composed of DOPC and DPPC. Domain formation and shape of these bilayers were evaluated in two fluid systems (chambers and slides). It was found that bilayers formed on conditioned glass, placed on a slide, produced highly detailed bilayers. Distinct lipid phase separation could be seen. Bilayers composed of 3:1 DOPC:DPPC with $0.8 \%$ mol TR-DHPE produced bilayers virtually absent of dye aggregates, and the best photostability. The information obtained in this work will assist in creating well-controlled environments for SLB to be studied via TIRFM. Future work will 
look at the application of membrane-targeting antimicrobials to these environments, to determine their actions on lipid phases.

\section{Acknowledgement}

The authors thank Justin Falardeau for technical assistance. This work was financially supported by grant RGPIN-2015-05679 from the Natural Sciences and Engineering Research Council (NSERC) of Canada to Tyler J. Avis. 


\subsection{References}

[1] S.J. Singer, G.L. Nicolson, The fluid mosaic model of the structure of cell membranes, Science, 175 (1972) 720-731.

[2] D.E. Green, A. Tzagoloff, Role of lipids in the structure and function of biological membranes, Journal of Lipid Research, 7 (1966) 587-602.

[3] Y.-H.M. Chan, S.G. Boxer, Model membrane systems and their applications, Current Opinion in Chemical Biology, 11 (2007) 581-587.

[4] H. Schönherr, J.M. Johnson, P. Lenz, C.W. Frank, S.G. Boxer, Vesicle adsorption and lipid bilayer formation on glass studied by atomic force microscopy, Langmuir, 20 (2004) 11600-11606. [5] D.M.C. Ramirez, W.W. Ogilvie, L.J. Johnston, NBD-cholesterol probes to track cholesterol distribution in model membranes, Biochimica et Biophysica Acta (BBA) - Biomembranes, 1798 (2010) 558-568.

[6] J.M. Vanegas, M.F. Contreras, R. Faller, M.L. Longo, Role of unsaturated lipid and ergosterol in ethanol tolerance of model yeast biomembranes, Biophysical Journal, 102 (2012) 507-516.

[7] C. Hamai, T. Yang, S. Kataoka, P.S. Cremer, S.M. Musser, Effect of average phospholipid curvature on supported bilayer formation on glass by vesicle fusion, Biophysical Journal, 90 (2006) 1241-1248.

[8] W.J. Galush, J.A. Nye, J.T. Groves, Quantitative fluorescence microscopy using supported lipid bilayer standards, Biophysical Journal, 95 (2008) 2512-2519.

[9] E. Kalb, S. Frey, L.K. Tamm, Formation of supported planar bilayers by fusion of vesicles to supported phospholipid monolayers, Biochimica et Biophysica Acta, 1103 (1992) 307-316.

[10] A.A. Brian, H.M. McConnell, Allogeneic stimulation of cytotoxic T cells by supported planar membranes, Proceedings of the National Academy of Sciences, 81 (1984) 6159-6163.

[11] P.S. Cremer, J.T. Groves, L.A. Kung, S.G. Boxer, Writing and erasing barriers to lateral mobility into fluid phospholipid bilayers, Langmuir, 15 (1999) 3893-3896.

[12] Ira, S. Zou, D. Ramirez, S. Vanderslip, W. Ogilvie, Z. Jakubek, L. Johnston, Enzymatic generation of ceramide induces membrane restructuring: correlated AFM and fluorescence imaging of supported bilayers, Journal of Structural Biology, 168 (2009) 78-89. 
[13] O.G. Travkova, G. Brezesinski, Adsorption of the antimicrobial peptide arenicin and its linear derivative to model membranes - A maximum insertion pressure study, Chemistry and Physics of Lipids, 167-168 (2013) 43-50.

[14] E.T. Castellana, P.S. Cremer, Solid supported lipid bilayers: from biophysical studies to sensor design, Surface Science Reports, 61 (2006) 429-444.

[15] G.J. Hardy, R. Nayak, S. Zauscher, Model cell membranes: techniques to form complex biomimetic supported lipid bilayers via vesicle fusion, Current Opinion in Colloid and Interface Science, 18 (2013) 448-458.

[16] A.E. McKiernan, T.V. Ratto, M.L. Longo, Domain growth, shapes, and topology in cationic lipid bilayers on mica by fluorescence and atomic force microscopy, Biophysical Journal, 79 (2000) 2605-2615.

[17] H.M. McConnell, T.H. Watts, R.M. Weis, A.A. Brian, Supported planar membranes in studies of cell-cell recognition in the immune system, Biochimica et Biophysica Acta (BBA) - Reviews on Biomembranes, 864 (1986) 95-106.

[18] F. Neville, M. Cahuzac, O. Konovalov, Y. Ishitsuka, K.Y.C. Lee, I. Kuzmenko, G.M. Kale, D. Gidalevitz, Lipid headgroup discrimination by antimicrobial peptide LL-37: insight into mechanism of action, Biophysical Journal, 90 (2006) 1275-1287.

[19] R.M. Epand, R.F. Epand, Lipid domains in bacterial membranes and the action of antimicrobial agents, Biochimica et Biophysica Acta, 1788 (2009) 289-294.

[20] A. Choucair, M. Chakrapani, B. Chakravarthy, J. Katsaras, L.J. Johnston, Preferential accumulation of $A \beta(1-42)$ on gel phase domains of lipid bilayers: an AFM and fluorescence study, Biochimica et Biophysica Acta, 1768 (2007) 146-154.

[21] S. Gromelski, A.M. Saraiva, R. Krastev, G. Brezesinski, The formation of lipid bilayers on surfaces, Colloids and Surfaces B: Biointerfaces, 74 (2009) 477-483.

[22] U. Seifert, R. Lipowsky, Adhesion of vesicles, Physical Review A, 42 (1990) 4768-4771.

[23] K. Dimitrievski, Deformation of adsorbed lipid vesicles as a function of vesicle size, Langmuir, 26 (2010) 3008-3011.

[24] B.A. Cornell, G.C. Fletcher, J. Middlehurst, F. Separovic, The lower limit to the size of small sonicated phospholipid vesicles, Biochimica et Biophysica Acta (BBA) - Biomembranes, 690 (1982) 15-19.

[25] D.D. Lasic, The mechanism of vesicle formation, Biochemical Journal, 256 (1988) 1-11. 
[26] J. Seelig, P.M. Macdonald, Phospholipids and proteins in biological membranes. Deuterium NMR as a method to study structure, dynamics, and interactions, Accounts of Chemical Research, 20 (1987) 221-228.

[27] J.J. De Vries, H.J.C. Berendsen, Nuclear magnetic resonance measurements on a macroscopically ordered smectic liquid crystalline phase, Nature, 221 (1969) 1139-1140.

[28] P.F.F. Almeida, Thermodynamics of lipid interactions in complex bilayers, Biochimica et Biophysica Acta, 1788 (2009) 72-85.

[29] T. Dewa, S.J. Vigmond, S.L. Regen, Lateral heterogeneity in fluid bilayers composed of saturated and unsaturated phospholipids, Journal of the American Chemical Society, 118 (1996) 3435-3440.

[30] J.E. Shaw, A. Slade, C.M. Yip, Simultaneous in situ total internal reflectance fluorescence/atomic force microscopy studies of DPPC/dPOPC microdomains in supported planar lipid bilayers, Journal of the American Chemical Society, 125 (2003) 11838-11839.

[31] S.L. Veatch, S.L. Keller, Seeing spots: complex phase behavior in simple membranes, Biochimica et Biophysica Acta (BBA) - Molecular Cell Research, 1746 (2005) 172-185.

[32] T. Baumgart, G. Hunt, E.R. Farkas, W.W. Webb, G.W. Feigenson, Fluorescence probe partitioning between Lo/Ld phases in lipid membranes, Biochimica et Biophysica Acta, 1768 (2007) 2182-2194.

[33] M.A. Holden, S.-Y. Jung, T. Yang, E.T. Castellana, P.S. Cremer, Creating fluid and air-stable solid supported lipid bilayers, Journal of the American Chemical Society, 126 (2004) 6512-6513. [34] J. Zhao, J. Wu, H. Shao, F. Kong, N. Jain, G. Hunt, G. Feigenson, Phase studies of model biomembranes: macroscopic coexistence of $L \alpha+L \beta$, with light-induced coexistence of $L \alpha+$ Lo Phases, Biochimica et Biophysica Acta, 1768 (2007) 2777-2786.

[35] S.L. Veatch, S.L. Keller, Separation of liquid phases in giant vesicles of ternary mixtures of phospholipids and cholesterol, Biophysical Journal, 85 (2003) 3074-3083.

[36] J. Yuan, C. Hao, M. Chen, P. Berini, S. Zou, Lipid reassembly in asymmetric langmuirblodgett/langmuir-schaeffer bilayers, Langmuir, 29 (2013) 221-227.

[37] T. Cha, A. Guo, X.Y. Zhu, Formation of supported phospholipid bilayers on molecular surfaces: role of surface charge density and electrostatic interaction, Biophysical Journal, 90 (2006) 1270-1274. 
[38] H. Bouvrais, T. Pott, L.A. Bagatolli, J.H. Ipsen, P. Méléard, Impact of membrane-anchored fluorescent probes on the mechanical properties of lipid bilayers, Biochimica et Biophysica Acta (BBA) - Biomembranes, 1798 (2010) 1333-1337.

[39] S.J. Attwood, Y. Choi, Z. Leonenko, Preparation of DOPC and DPPC supported planar lipid bilayers for atomic force microscopy and atomic force spectroscopy, International Journal of Molecular Science, 14 (2013) 3514-3539.

[40] D. Axelrod, Cell-substrate contacts illuminated by total internal reflection fluorescence, The Journal of Cell Biology, 89 (1981) 141-145.

[41] A.L. Mattheyses, S.M. Simon, J.Z. Rappoport, Imaging with total internal reflection fluorescence microscopy for the cell biologist, Journal of Cell Science, 123 (2010) 3621-3628. [42] M.A. Ravier, T. Tsuboi, G.A. Rutter, Imaging a target of Ca2+ signalling: Dense core granule exocytosis viewed by total internal reflection fluorescence microscopy, Methods, 46 (2008) 233238.

[43] S. Hageneder, M. Bauch, J. Dostalek, Plasmonically amplified bioassay - Total internal reflection fluorescence vs. epifluorescence geometry, Talanta, 156-157 (2016) 225-231.

[44] J. Oreopoulos, C.M. Yip, Combinatorial microscopy for the study of protein-membrane interactions in supported lipid bilayers: Order parameter measurements by combined polarized TIRFM/AFM, Journal of Structural Biology, 168 (2009) 21-36.

[45] L.A. Bagatolli, To see or not to see: lateral organization of biological membranes and fluorescence microscopy, Biochimica et Biophysica Acta (BBA) - Biomembranes, 1758 (2006) 1541-1556.

[46] D. Chen, M.M. Santore, 1,2-Dipalmitoyl-sn-glycero-3-phosphocholine (DPPC)-rich domain formation in binary phospholipid vesicle membranes: two-dimensional nucleation and growth, Langmuir, 30 (2014) 9484-9493.

[47] P.S. Cremer, S.G. Boxer, Formation and spreading of lipid bilayers on planar glass supports, Journal of Physical Chemistry B, 103 (1999) 2554-2559.

[48] Ira, L.J. Johnston, Sphingomyelinase generation of ceramide promotes clustering of nanoscale domains in supported bilayer membranes, Biochimica et Biophysica Acta (BBA) - Biomembranes, 1778 (2008) 185-197.

[49] S.M. Polyakova, V.N. Belov, S.F. Yan, C. Eggeling, C. Ringemann, G. Schwarzmann, A. de Meijere, S.W. Hell, New GM1 Ganglioside Derivatives for Selective Single and Double Labelling 
of the Natural Glycosphingolipid Skeleton, European Journal of Organic Chemistry, 2009 (2009) 5162-5177.

[50] P.S. Uster, R.E. Pagano, Resonance energy transfer microscopy: observations of membranebound fluorescent probes in model membranes and in living cells, The Journal of Cell Biology, 103 (1986) 1221-1234.

[51] D. Dulin, A. Le Gall, K. Perronet, N. Soler, D. Fourmy, S. Yoshizawa, P. Bouyer, N. Westbrook, Reduced photobleaching of BODIPY-FL, Physics Procedia, 3 (2010) 1563-1567.

[52] N.L. Thompson, K.H. Pearce, H.V. Hsieh, Total internal reflection fluorescence microscopy: application to substrate-supported planar membranes, European Biophysics Journal, 22 (1993) 367-378.

[53] T. Kaasgaard, C. Leidy, J.H. Crowe, O.G. Mouritsen, K. Jørgensen, Temperature-controlled structure and kinetics of ripple phases in one- and two-component supported lipid bilayers, Biophysical Journal, 85 (2003) 350-360.

[54] A.R. Honerkamp-Smith, S. Veatch, S.L. Keller, An introduction to critical points for biophysicists; observations of compositional heterogeneity in lipid membranes, Biochimica et Biophysica Acta, 1788 (2009) 53-63.

[55] S. Daniel, A.J. Diaz, K.M. Martinez, B.J. Bench, F. Albertorio, P.S. Cremer, Separation of membrane-bound compounds by solid-supported bilayer electrophoresis, Journal of the American Chemical Society, 129 (2007) 8072-8073.

[56] J. Juhasz, J. Davis, F. Sharom, Fluorescent probe partitioning in giant unilamellar vesicles of 'lipid raft' mixtures, Biochemical Journal, 430 (2010) 415-423.

[57] M.J. Skaug, M.L. Longo, R. Faller, The impact of texas red on lipid bilayer properties, The Journal of Physical Chemistry B, 115 (2011) 8500-8505.

[58] Y. Kashimura, J. Durao, K. Furukawa, T. Keiichi, Self-spreading behavior of supported lipid bilayer through single sub-100-nm gap, Japanese Journal of Applied Physics, 47 (2008) 3248.

[59] Y. Kashimura, K. Furukawa, T. Keiichi, Self-spreading supported lipid bilayer passing through single nanogap structure: effect of position of dyes in lipid molecules, Japanese Journal of Applied Physics, 49 (2010) 04DL15.

[60] P. Somerharju, J.A. Virtanen, K.H. Cheng, M. Hermansson, The superlattice model of lateral organization of membranes and its implications on membrane lipid homeostasis, Biochimica et Biophysica Acta (BBA) - Biomembranes, 1788 (2009) 12-23. 
[61] L.M.S. Loura, J.P.P. Ramalho, Recent developments in molecular dynamics simulations of fluorescent membrane probes, Molecules, 16 (2011) 5437-5452.

[62] L.J. Johnston, Nanoscale imaging of domains in supported lipid membranes, Langmuir, 23 (2007) 5886-5895.

[63] N.L. Thompson, X. Wang, P. Navaratnarajah, Total internal reflection with fluorescence correlation spectroscopy: applications to substrate-supported planar membranes, Journal of Structural Biology, 168 (2009) 95-106. 


\title{
Chapter 3
}

Domain redistribution within ergosterol-containing model membranes in the presence of the antimicrobial compound fengycin

\author{
Elisabeth Mantila ${ }^{\mathrm{a}}$, Trinda Crippina, Tyler J. Avis ${ }^{\mathrm{a}, \mathrm{b}, *}$
}

${ }^{1}$ Department of Chemistry, Carleton University. 1125 Colonel By Drive, Ottawa, ON K1S 5B6,

Canada

${ }^{2}$ Institute of Biochemistry, Carleton University, 1125 Colonel By Drive, Ottawa, ON K1S 5B6,

Canada 


\section{Abstract}

The cyclic lipopeptide fengycin, produced by Bacillus subtilis, exhibits its antimicrobial capabilities by altering the integrity of the cell membrane of plant pathogens. Previous work has correlated fengycin activity with membrane characteristics, such as sterol content. This work focused on the influence of fengycin on supported lipid bilayers containing varying levels of ergosterol. Total internal reflection fluorescence (TIRF) microscopy was used to visualize and distinguish ordered $\left(\mathrm{L}_{\mathrm{o}}\right)$ and disordered $\left(\mathrm{L}_{\mathrm{d}}\right)$ domains in the model membranes following exposure to low $(50 \mu \mathrm{g})$ and high $(500 \mu \mathrm{g})$ fengycin doses. Application of a low dose of fengycin to $0 \%$ and $3 \%$ ergosterol-containing bilayers resulted in redistribution of $\mathrm{L}_{o}$ and $\mathrm{L}_{d}$ domains, which the bilayers compensated and corrected for over time. These membranes were unable to tolerate a second $50 \mu \mathrm{g}$ dose or the high fengycin dose. The $6 \%$ ergosterol bilayers were able to tolerate sequential low doses of fengycin. Exposure of these bilayers to the high fengycin dose caused a decrease in the number of $\mathrm{L}_{\mathrm{o}}$ domains, albeit less than that seen in the $0 \%$ and $3 \%$ ergosterol bilayers. Bilayers containing $12 \%$ ergosterol, exhibited the least amount of change after fengycin exposure. These were the only bilayer to exhibit an increase in area taken up by ordered domains. These results suggest fengycin may preferentially act on the $\mathrm{L}_{\mathrm{o}}$ phase, the area in which ergosterol resides. Bilayers containing low levels of ergosterol appear to be more sensitive to the lipopeptide, suggesting ergosterol plays a role in buffering perturbations caused by fengycin.

Keywords

Fengycin, antimicrobial compound, ergosterol, model membranes, domain distribution 


\subsection{Introduction}

The cell membrane is an integral part to the growth and development of a microorganism [1, 2]. It is responsible for protecting the contents of the cell from the external environment while maintaining a semi-permeable nature $[3,4]$. With respect to fungi, compounds that target the fungal membrane are of great interest when combatting human, animal, and plant mycoses. Such antifungal compounds include azoles, polyenes, and peptides.

Azoles interfere with the biosynthesis of the main fungal sterol, ergosterol, leading to a reduction in ergosterol production and an accumulation of lanosterol and 14-methylated sterols in the plasma membrane [5-7]. Azoles inhibitory effects can be attributed to their binding to cytochrome $\mathrm{P} 450$ proteins, such as cytochrome P450-Erg11p or Cyp51p. The efficacy of a specific azole is dependent on its activity and the P450 it is bound to [5, 8]. Impairment in ergosterol availability affects the overall integrity of the plasma membrane, making it vulnerable to additional damage.

Conversely, polyenes tend to target specific membrane components and the presence or absence of that component is directly related to its efficacy. Examples include amphotericin B $(\mathrm{AmpB})$, natamycin, and nystatin, which are gold standard antifungal agents with activity related to the presence of ergosterol in fungal membranes. AmpB and nystatin are known to mediate ion channel membrane permeabilization with ergosterol binding and are strongly influenced by the presence of the sterol. Binding of ergosterol influences its increased efficacy in fungal cells versus human cells [9-11]. Natamycin also binds directly to ergosterol but does not induce pore formation. The binding of natamycin to ergosterol blocks membrane protein transport functionality [12, 13].

Focus has shifted from the use of common synthetic fungicidal agents to antimicrobial peptides, in an effort to reduce the potential for resistance development [14]. Antimicrobial 
peptides (AMPs) are attractive antimicrobial agents due to their broad spectrum of activity against microbes, as a result of having a more generalized effect on membranes versus other antimicrobials, which target specific biochemical pathways [15]. Having a generalized effect means they may act on the membrane in a number of manners such as influencing membrane thickness, membrane polarization and lipid charge, and/or introducing pore formation $[15,16]$.

Among other membrane-targeting antimicrobials, fengycins, cyclic lipopeptides produced primarily by bacteria of the Bacillus genus, have gained attention as potential antifungal agents [17-20], specifically against fungal plant pathogens sharing the same ecological niche as these bacteria [21]. The structure of fengycin, a lipodecapeptide, consists of an internal lactone ring within the peptide moiety along with a $\beta$-fatty acid chain (containing 14 to 18 carbons) that can be saturated or unsaturated $[19,22]$. Fengycin has been shown to act in an all-or-none fashion rather than in a graded manner, such as that shown by amphotericin B and other polyene antimycotics, albeit the full mechanism of fengycin has not been elucidated $[23,24]$. It has been shown that fengycin readily interacts with the lipid bilayer, but the specific interaction(s) within the membrane are not fully understood. Fengycin has exhibited antimicrobial activity against numerous plant pathogens through alteration of the integrity of the cell membranes [25]. These interactions lead to increased permeability and pore formation within the bilayer $[17,26]$.

Previous work indicated that membrane lipid content was a main determinant in the sensitivity of phytopathogens to fengycin. More specifically, fungal membranes containing lower levels of ergosterol were more sensitive to fengycin [25]. In liposome leakage assays, liposome mixtures containing sterols inhibited leakage caused by fengycin [27]. In these assays, ergosterol was less effective than cholesterol when using a $30 \mathrm{~mol} \%$ concentration and the presence of sterols did not impact the all-or-none action of fengycin [27]. Ergosterol has been shown to buffer fluidity 
changes in the presence of other membrane-targeting antimicrobials [28], suggesting a similar process may be happening in the presence of fengycin.

In the present study, supported lipid bilayers (SLBs) were used to investigate the effects of fengycin on membranes. While SLBs are not as complex as lipid membranes found in nature, they are useful models for the study of interactions at the bilayer surface [29-31]. SLBs can be composed of a variety of lipids varying in overall charge, degree of saturation, and molar ratio, as summarized in previous work [32].

Earlier studies have shown that SLBs composed of a 3:1 molar ratio of 1,2-dioleoyl-snglycero-3-phosphocholine (DOPC) to 1,2-dipalmitoyl-sn-glycero-3-phosphocholine (DPPC) with $0.8 \quad \operatorname{mol} \% \quad$ Texas $\quad \operatorname{Red}{ }^{\circledR} \quad$ 1,2-dihexadecanoyl-sn-glycero-3-phosphoethanolamine triethylammonium salt (TR-DHPE) were an appropriate system to study membrane characteristics using total internal reflection fluorescence (TIRF) microscopy [32]. In the present work, these SLB were used to perform a TIRF microscopy investigation on the effect of varying levels of ergosterol in membranes exposed to fengycin. The distribution and characteristics of the lipid phases (ordered and disordered) were evaluated.

\subsection{Materials and Methods}

\subsubsection{Materials}

1,2-Dioleoyl-sn-glycero-3-phosphocholine (DOPC) and 1,2-dipalmitoyl-sn-glycero-3phosphocholine (DPPC) were purchased from Avanti Polar Lipid (Alabaster, AL). HPLC-grade ( $\geq 95 \%$ ) ergosterol was obtained from Sigma-Aldrich (Oakville, ON). Texas Red® 1,2dihexadecanoyl-sn-glycero-3-phosphoethanolamine triethylammonium salt (TR-DHPE) was 
obtained from Life Technologies (Burlington, ON). Reagent grade chloroform was obtained from Caledon Laboratories (Georgetown, ON). The lipid solution buffer used in all experiments contained BioPerformance Certified ( $\geq 99.0 \%)$ Trizma ${ }^{\circledR}$ hydrochloride (Tris-HCl) and calcium chloride $\left(\mathrm{CaCl}_{2}\right)$ from Sigma-Aldrich, sodium chloride $(\mathrm{NaCl})$ from BioShop Canada (Burlington, ON), and Milli-Q water deionized to a resistivity of $18.2 \mathrm{M} \Omega \cdot \mathrm{cm}$. Glass coverslips (\#1.5) from Warner Instruments (Hamden, CT) were conditioned by placing in a boiling Sparkleen (Fisher Scientific, Ottawa, ON) solution for ten minutes, followed by washing with copious amounts of distilled water for 20 minutes. After rinsing, coverslips were dried in a $343^{\circ} \mathrm{C}$ kiln for a minimum of six hours. Fengycin was produced and purified from Bacillus subtilis (Ehrenberg) Cohn as described previously $[17,33]$.

\subsubsection{Vesicle preparation}

Lipid solutions were prepared as previously described [32]. Briefly, lipid stock solutions were prepared, using chloroform, in glass vials. Aliquots of stock solutions were used to obtain solutions with a DOPC:DPPC molar ratio of 3:1 solutions in which ergosterol was added to obtain concentrations of $0,3,6$, and $12 \mathrm{~mol} \%$ ergosterol. All lipid solutions contained $0.8 \mathrm{~mol} \%$ TRDHPE. Chloroform was evaporated under vacuum for a minimum of one hour or until all traces of solvent were removed. The lipid films were rehydrated with SLB buffer $\left(3 \mathrm{mM} \mathrm{CaCl}_{2}, 10 \mathrm{mM}\right.$ Tris- $\mathrm{HCl}, 100 \mathrm{mM} \mathrm{NaCl} ; \mathrm{pH} 7)$, to a final concentration of $0.75 \mathrm{mg}$ lipids $/ \mathrm{ml}$, and rotated for one hour in a water bath of $80^{\circ} \mathrm{C}$. The hydrated lipid solution was then sonicated for one hour at $80^{\circ} \mathrm{C}$ (Codyson PS-20 Ultrasonic Cleaner, AC 110-120V, 50-60 Hz). Following sonication, lipid solutions were extruded through a polycarbonate membrane $(0.05 \mu \mathrm{m})$ via gas tight glass syringes, according to manufacturer's instructions (Avanti Polar Lipids). The extrusion apparatus was kept 
at $60^{\circ} \mathrm{C}$ during the extrusion process. Solutions were brought to room temperature and stored at $4^{\circ} \mathrm{C}$ for up to four days prior use.

\subsubsection{Bilayer formation}

Supported lipid bilayers were prepared via the vesicle fusion method on slides, as previously described $[30,32,34]$. Briefly, $75 \mu \mathrm{L}$ of vesicle solution was aliquoted into a crystallization dish and a conditioned coverslip was placed over top. The coverslip was then incubated at $60^{\circ} \mathrm{C}$ for 10 minutes. After incubation, a two-inch layer of Milli-Q water was poured over top of the coverslip. Working within the water layer, the coverslip was lifted and shaken to remove excess lipids. The coverslip was then transferred to a slide, using double sided tape along the edges to anchor the coverslip. The slide was removed from the incubation dish and excess water was eliminated.

\subsubsection{Fengycin addition to SLBs}

After forming each SLB on a slide, initial images were taken using the TIRF microscope as described below. After initial imaging, $50 \mu \mathrm{g}$ of fengycin was pipetted into the bilayer along the edge of the slide. Images were taken immediately after the addition of fengycin and labelled as “time 0". Sequential images were taken every minute for the first five minutes. Images were again taken after 20 and 40 minutes of incubation. A second addition of $50 \mu \mathrm{g}$ of fengycin was performed after the 40 minute incubation. Again, images were taken every minute for the first five minutes, and again after 20 and 40 minutes of incubation. This experiment was also performed with a single dose of $500 \mu \mathrm{g}$ of fengycin, as well as the same volume of MilliQ water (control). The experiments were performed three times. 


\subsubsection{TIRF Imaging}

An Olympus IX83 inverted microscope (Richmond Hill, ON), equipped with 405 and $473 \mathrm{~nm}$ diode lasers, a $568 \mathrm{~nm}$ diode-pumped solid state (DPPS) laser, and $60 \times$ and $100 \times$ TIRF oil immersion objectives, was used to image all supported lipid bilayers. Z-Drift compensation was enabled on the cellTIRF-4Line system (Olympus). TR-DHPE was excited with the $568 \mathrm{~nm}$ laser, separately, with emissions collected using a LF561 cube. Images were collected using a Rolera EM-C ${ }^{2}$ camera (Qimaging, Surrey, BC). MetaMorph ${ }^{\circledR}$ Advanced version 7.8.2.0 software (Molecular Devices, Sunnyvale, CA) and ImageJ were used to analyze all images. Data obtained from each time point were converted to an 8-bit, thresholded image. Using these images, total membrane domains absent of fluorescence (lower 25\%) and those containing above average levels of fluorescence (upper 15\%) were outlined and enumerated. Images from three independent trials were analyzed for each data point and averaged. Individual total domain area was also measured. Domains absent of fluorescence were characterized as ordered domains $\left(\mathrm{L}_{\mathrm{o}}\right)$, consisting primarily of DPPC and ergosterol. Preferential portioning of TR-DHPE into disordered domains $\left(\mathrm{L}_{\mathrm{d}}\right)$, containing DOPC, was responsible for the above average levels of fluorescence. This data processing was then repeated on domains of specific sizes. In this case, sizes were defined as small $(<1 \mu \mathrm{m})$, medium $(1-10 \mu \mathrm{m})$, and large $(>10 \mu \mathrm{m})$ domains, as described previously [32]. After converting the TIRFM image to an 8-bit image, inverting and thresholding, domains were measured using the function Analyze Particles. Briefly, as the image was scanned, domain edges were detected and outlined by the software and when measured was filled to make it colourless. Area was calculated based on the number of pixels counted within each domain $\left(\right.$ pixel $\left.^{2}\right)$ and converted to $\mu \mathrm{m}^{2}$. Percent area was calculated as the number of pixels counted in the highlighted 
fraction (domains of interest), in comparison to the total pixel count of the image. Images outside the range of circularity were ignored. Circularity was defined as: Circularity $=4 \pi \times$ (area/perimeter ${ }^{2}$ ), where circularity was a set range of 0.00 to $1.00,1.00$ indicating a perfect circle and values closer to 0.00 indicates an elongated shape. Data was reported as the relative count, size, and area of the domains following addition of the initial fengycin dose.

\subsection{Results}

\subsubsection{Ordered $\left(\mathrm{L}_{\mathrm{o}}\right)$ and disordered $\left(\mathrm{L}_{\mathrm{d}}\right)$ domain counts}

\subsubsection{Low fengycin doses}

Following an initial injection of $50 \mu \mathrm{g}$ of fengycin into 3:1 DOPC:DPPC bilayers without ergosterol, an increase in the number of $\mathrm{L}_{\mathrm{o}}$ domains (Fig. 3.1A) could be seen after two minutes. The domain distribution immediately returned to its original state, and remained stable until the 40-minute mark. Following the second $50 \mu \mathrm{g}$ dose, there was an increase in counts, which reduces by half 20 minutes later ( 61 mins mark), before increasing again. This trend was mimicked in the small $(<1 \mu \mathrm{m})$ (Fig. S1A) and medium $(1-10 \mu \mathrm{m})$ sized domains (Fig. S1D). Overall, there were 2-fold more domains at the end of the incubation period and this trend can be seen directly in the 2-fold increase of medium sized domains (Fig. S1D). There was a 14-fold increase in small domains (Fig. S1A). Little change in the large sized $(>10 \mu \mathrm{m})$ domains was seen. 

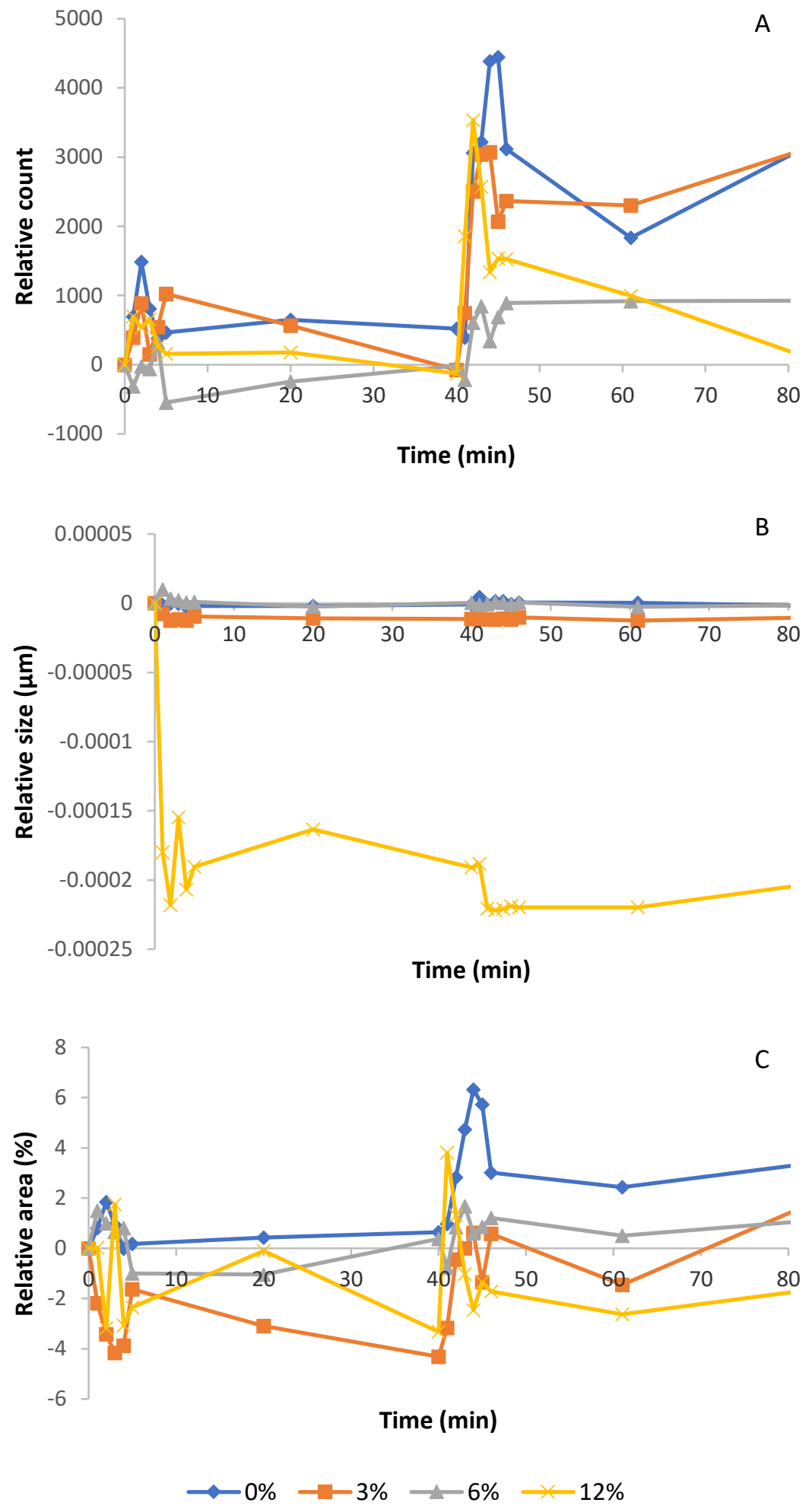

Figure 3.1. Effect of low doses $(50 \mu \mathrm{g})$ of fengycin on liquid ordered $\left(\mathrm{L}_{\mathrm{o}}\right)$ domain characteristics and distribution of lipid bilayers. Bilayers contained 3:1 DOPC:DPPC with 0 (blue), 3 (orange), 6 (grey), and $12 \%$ (yellow) ergosterol. Fengycin added at the 0 minute (dose 1) and the 40 minute marks (dose 2). A. Relative $\mathrm{L}_{0}$ domain count; B. relative $\mathrm{L}_{0}$ domain size; C. relative $\mathrm{L}_{0}$ domain area. 
The $\mathrm{L}_{\mathrm{d}}$ domains followed the same trend seen in the $\mathrm{L}_{0}$ domains (Fig. 3.2A), albeit only with a $50 \%$ increase in the overall domain count. Similarly, small $\mathrm{L}_{\mathrm{d}}$ domains resembled this overall trend, with a five-time increase at the second addition and remained 3-4 times higher than its initial state (Fig. S2A). Counts of the medium sized domains fluctuated, with a final count approximately a third of that recorded at time zero (Fig. S2D).

Bilayers containing $3 \%$ ergosterol had a similar number of $\mathrm{L}_{\mathrm{o}}$ domains as the $0 \%$ ergosterol bilayers (Fig. 3.1A). While counts fluctuated, the final count was approximately 3 -fold than at the beginning of the experiment (Fig. 3.1A). This increase was primarily related to the 3 -fold increased presence of small sized domains (Fig. S1A). The $\mathrm{L}_{d}$ domains followed a trend similar to that in the 0\% ergosterol trials (Fig. 3.2A and C). A 3-fold increase in $L_{d}$ domain count was seen (Fig. 3.2A) after incubation, more specifically in the small domain counts (Fig. S2A).

The $\mathrm{L}_{0}$ count trend in the $6 \%$ ergosterol trials, remained similar to its original count at $\mathrm{T}_{0}$. The $6 \%$ ergosterol trials had an ordered domain count, which was one third that of the counts seen in the 0 and 3\% trials (Fig. 3.1A). Like all other treatments, the 6\% ergosterol treatment saw an increase in overall $\mathrm{L}_{o}$ domain count, but unlike the other treatments, remained high. The final count was a third of that seen on the $0 \%$ and $3 \%$ ergosterol trials. The small and medium domain counts resembled those seen in the $0 \%$ and $3 \%$ ergosterol trials (Fig. S1A and D). The small domain count remained similar after the second dosage, whereas a decrease was seen in all other trials (Fig. S1A). After the first exposure to fengycin, there was a decrease in $L_{d}$ domains (Fig. 3.2A). The count was approximately the same as the $0 \%$ ergosterol trials and a third of that of the $3 \%$ ergosterol trials (Fig. 3.2A). 


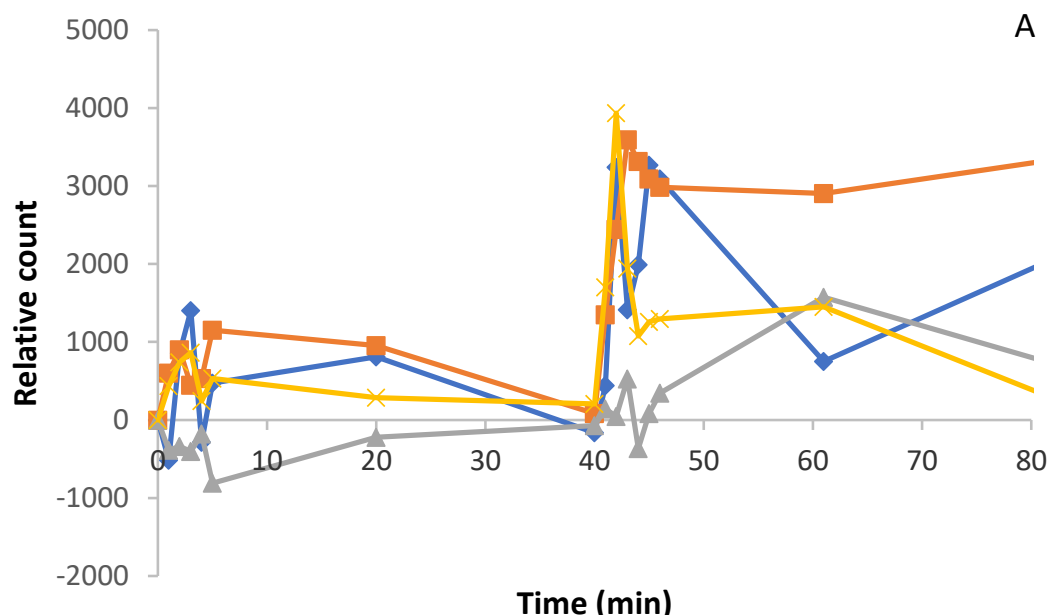

Time (min)

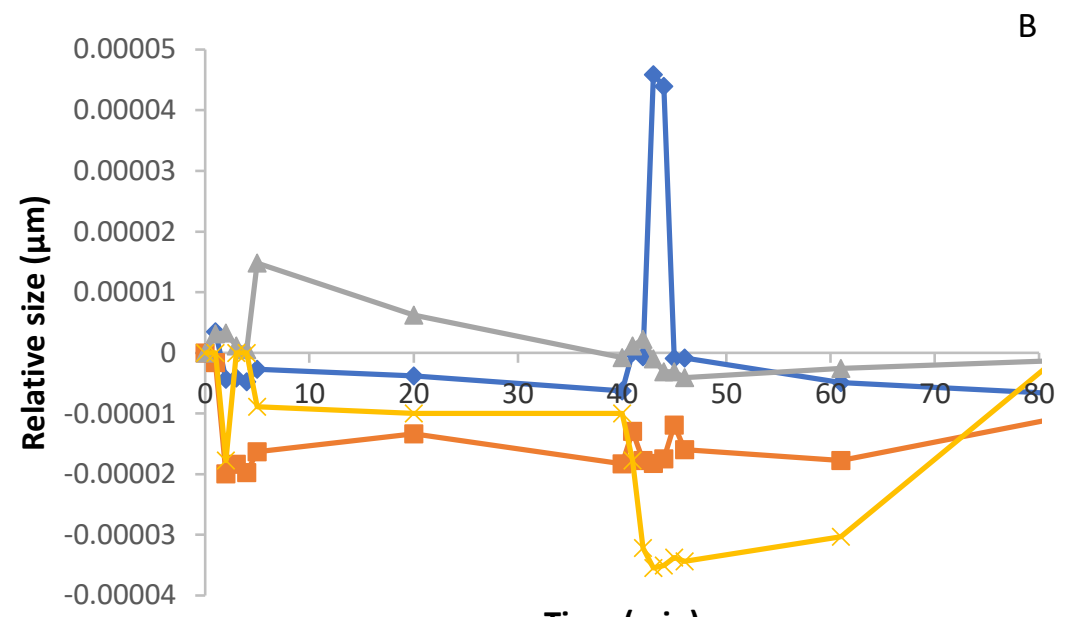

Time ( $\min )$

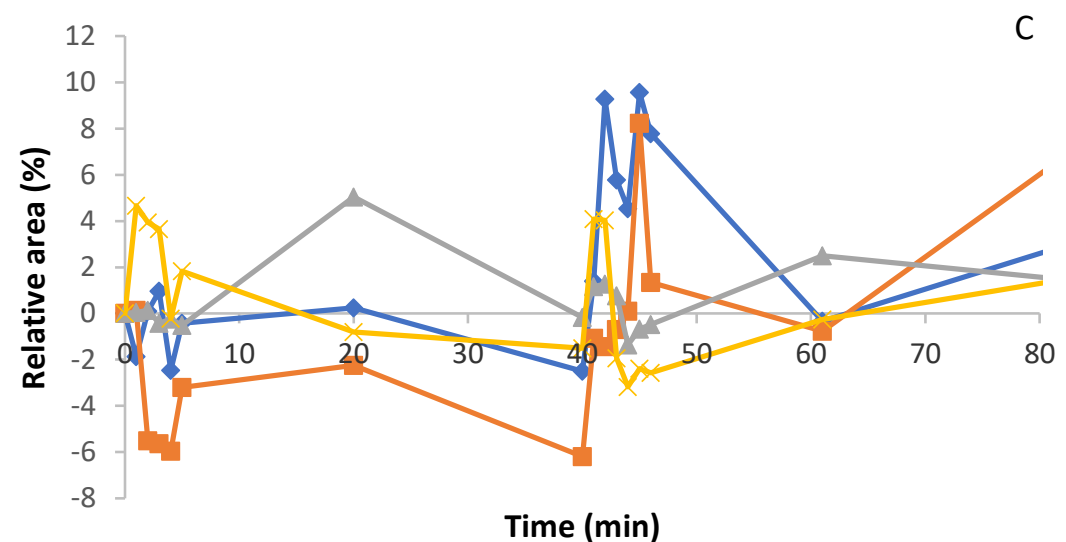

$\multimap 0 \%-3 \% \multimap-6 \% \multimap-12 \%$

Figure 3.2. Effect of low doses $(50 \mu \mathrm{g})$ of fengycin on liquid disordered $\left(\mathrm{L}_{\mathrm{d}}\right)$ domain characteristics and distribution of lipid bilayers. Bilayers contained 3:1 DOPC:DPPC with 0 (blue), 3 (orange), 6 (grey), and $12 \%$ (yellow) ergosterol. Fengycin added at the 0 minute (dose 1) and the 40 minute marks (dose 2). A. Relative $\mathrm{L}_{d}$ domain count; B. relative $\mathrm{L}_{d}$ domain size; C. relative $\mathrm{L}_{d}$ domain area. 
After incubation of the $12 \%$ ergosterol bilayers with $50 \mu \mathrm{g}$ of fengycin, approximately the same number of $\mathrm{L}_{\mathrm{o}}$ domains were observed as at time zero, although there was a significant increase in the first 5 minutes, similar to the 3\% ergosterol trials (Fig. 3.1A). By the second dose point (40 minutes), the count had returned to its original state. The addition of a second dose caused an increase in $\mathrm{L}_{\mathrm{o}}$ counts similar to that seen in the $3 \%$ ergosterol trials. Of particular interest, the $12 \%$ ergosterol treatments were the only ones showing a return to their original counts. While little to no large domains were seen in the other treatment groups, the $12 \%$ ergosterol trials showed an immediate $50 \%$ increase in these numbers, continuing to increase before settling with the $50 \%$ increase (Fig. S1G). Medium sized $\mathrm{L}_{0}$ domain counts followed the trend seen in the $3 \%$ ergosterol trial albeit with a decrease seen in the last 20 minutes of the incubation period (Fig. S1D). The same trend was seen in the small sized domain counts (Fig. S1A). Compared to the other treatment groups the $L_{d}$ count exhibited the smallest growth in domain counts (Fig. 3.2A). The most significant changes happened after the second fengycin dose. The $12 \%$ ergosterol trials followed trends similar to the $0 \%$ and $3 \%$ ergosterol trends for the first 40 minutes. The spike in counts seen at the second dose applications resembled the $3 \%$ ergosterol trial and immediately fell to approximately its original level by the end of the incubation period (Fig. 3.2A).

\subsubsection{High fengycin dose}

A dose of $500 \mu \mathrm{g}$ of fengycin was injected once and domain distribution was evaluated over a 40-minute period. In bilayers absent of ergosterol, there was a large increase in overall $\mathrm{L}_{\mathrm{o}}$ domain count (approximately 500\%) (Fig. 3.3A). The $\mathrm{L}_{0}$ count returned to its original state by five minutes and remained stable for the rest of the incubation period. Furthermore, there was a decrease in the number of large sized $\mathrm{L}_{\mathrm{o}}$ domains (Fig. $\mathrm{S} 3 \mathrm{G}$ ), and a slight increase in the medium domain count 

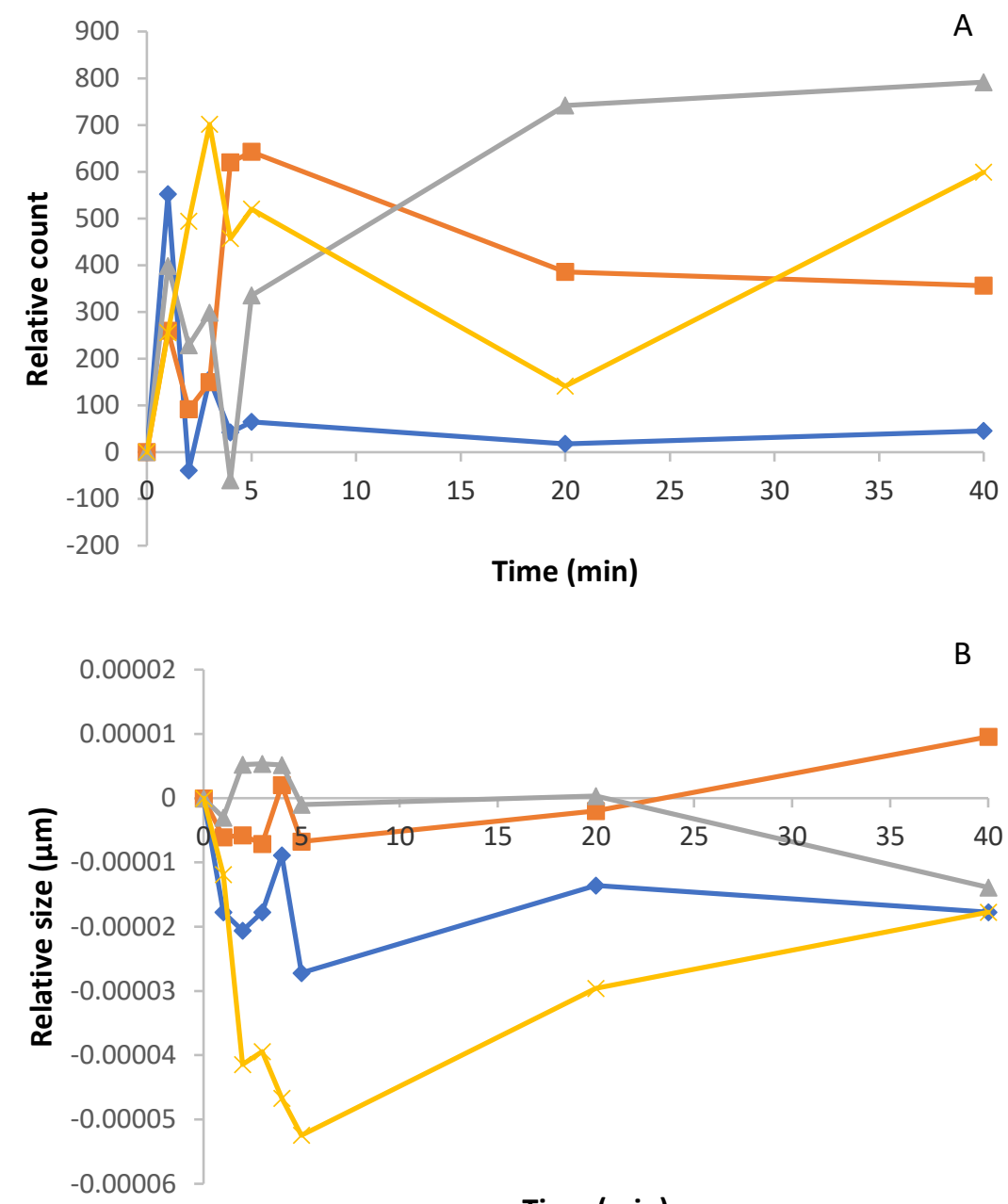

Time (min)

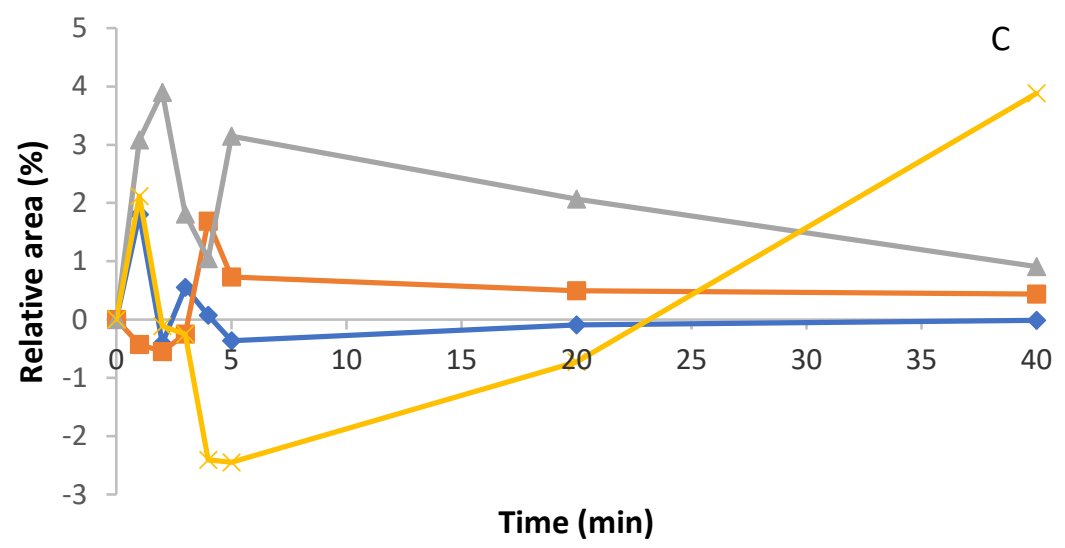

$\neg 0 \% \multimap-3 \% \multimap 6 \% \multimap 12 \%$

Figure 3.3. Effect of a high dose $(500 \mu \mathrm{g})$ of fengycin on liquid ordered $\left(\mathrm{L}_{\mathrm{o}}\right)$ domain characteristics and distribution of lipid bilayers. Bilayers contained 3:1 DOPC:DPPC with 0 (blue), 3 (orange), 6 (grey), and $12 \%$ (yellow) ergosterol. Fengycin added at the 0 minute mark. A. Relative $\mathrm{L}_{\mathrm{o}}$ domain count; B. relative $\mathrm{L}_{\mathrm{o}}$ domain size; C. relative $\mathrm{L}_{\mathrm{o}}$ domain area. 
while the small domain count remained the same (Figs. S3A and D). Fewer changes were found amongst the $\mathrm{L}_{\mathrm{d}}$ domains. An increase in domain count happened at three minutes, after which there was a gradual decrease, hovering around zero (Figs. 3.4A and C). Slight fluctuations were seen in the large and small sized $\mathrm{L}_{d}$ domains, whereas the largest difference was the almost 10-fold decrease in the medium sized domains (Fig. S4D).

Similar the $0 \%$ ergosterol bilayers, bilayers containing 3\% ergosterol saw an increase in the ordered domain count at the first minute, albeit only half the increase seen in the $0 \%$ ergosterol bilayers, with a second spike seen at four and five minutes (Fig. 3.3A). After five minutes, there was a gradual decrease in the counts, which was more rapid within the first 20 minutes of the incubation period. The $\mathrm{L}_{d}$ domains followed a similar trend in counts as the $0 \%$ ergosterol trials, with larger increases in counts within the first five minutes of dosing (Fig. 3.4A). The counts hovered around the initial count for the first 20 minutes before increasing $100 \%$ at 40 minutes. Severe fluctuations were seen amongst the small $\mathrm{L}_{\mathrm{d}}$ domain counts, with an approximate $600 \%$ increase within the first five minutes, which decreased by two-thirds after 20 minutes (Fig. 4SA). Their counts continued to decrease over time.

The $6 \%$ ergosterol trials mimicked the $0 \%$ and $3 \%$ ergosterol trials within the first five minutes. At four minutes there was a decrease in the $\mathrm{L}_{o}$ counts, but steadily began to increase ( $800 \%$ growth) throughout the remainder of the incubation period (Fig. 3.3A). A similar trend was seen in the $\mathrm{L}_{\mathrm{d}}$ domains, but the growth in counts plateaued after five minutes (Fig. 3.4A). Overall a $300 \%$ growth in counts was seen. 

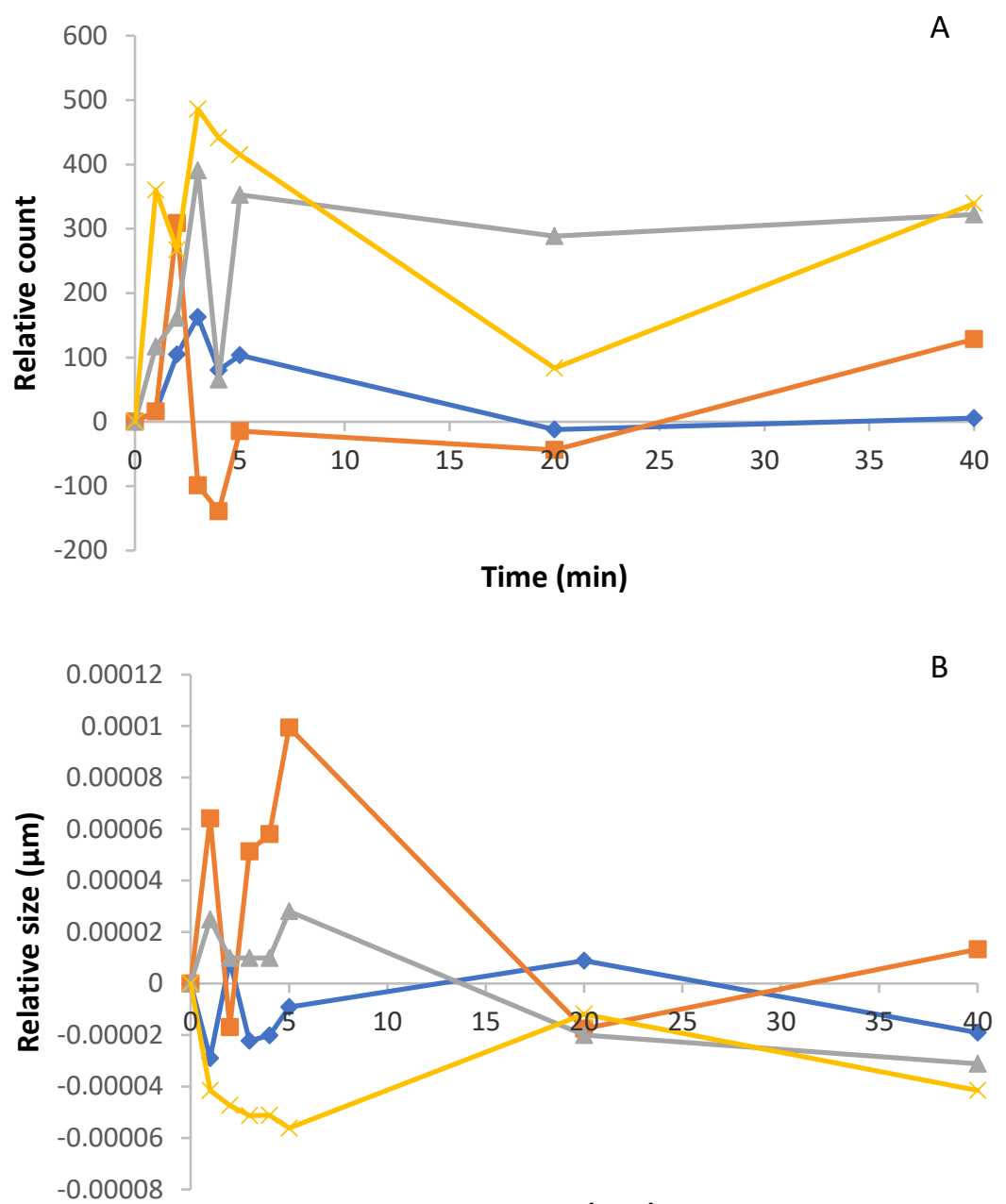

Time (min)

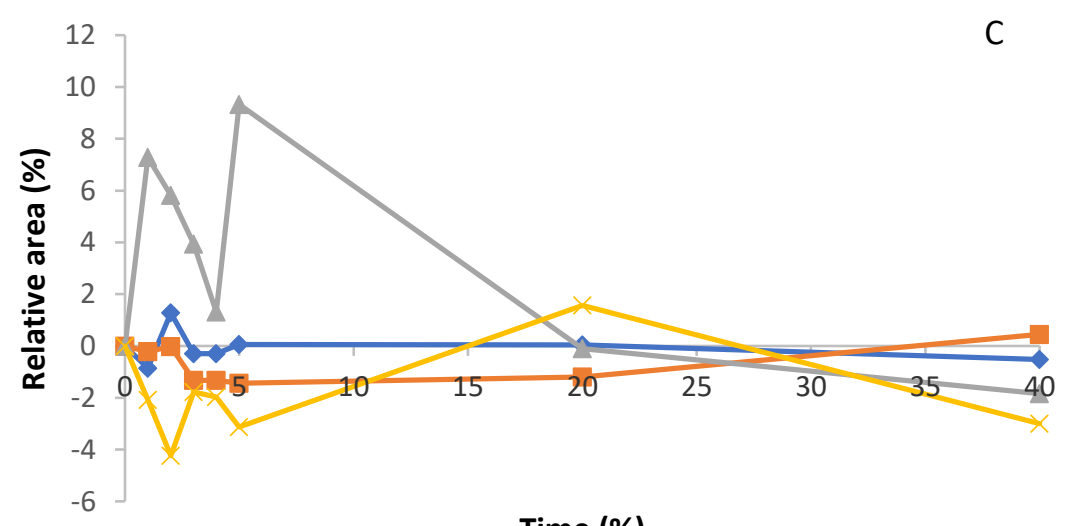

Time (\%)

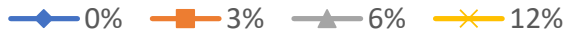

Figure 3.4. Effect of a high dose $(500 \mu \mathrm{g})$ of fengycin on liquid disordered $\left(\mathrm{L}_{\mathrm{d}}\right)$ domain characteristics and distribution of lipid bilayers. Bilayers contained 3:1 DOPC:DPPC with 0 (blue), 3 (orange), 6 (grey), and $12 \%$ (yellow) ergosterol. Fengycin added at the 0 minute mark. A. Relative $\mathrm{L}_{\mathrm{d}}$ domain count; $\mathrm{B}$. relative $\mathrm{L}_{\mathrm{d}}$ domain size; $\mathrm{C}$. relative $\mathrm{L}_{\mathrm{d}}$ domain area. 
The $12 \%$ ergosterol trials exhibited a similar trend seen within the first five minutes of all other treatment groups. Like the $0 \%$ and $3 \%$ ergosterol groups, there was a decrease in $\mathrm{L}_{o}$ counts after five-minutes, but counts rebounded by the end of the incubation period (Fig. 3.3A). This trend was seen amongst all domain size groupings, with a 70-fold increase in the medium domain final counts (Fig. S3D). The 12\% ergosterol trials experienced an immediate decrease in the large domain counts after fengycin application (Fig. S3G). There was an overall 3-fold decrease in the number of large sized domains present in the bilayer. In the $L_{d}$ domains, there was a faster, more immediate increase in the domain counts (highest of all trials), which immediately began to return to its initial state (Fig. 3.4A), gradually increasing again back to the initial spike level. Both the small and medium sized $\mathrm{L}_{\mathrm{d}}$ domains showed the largest increase in counts with a drastic increase in the first 20 minutes of the incubation period (Fig. S4A and D). The large domain counts had an opposite trend; there was an 8-fold decrease in the number of domains (Fig. S4G). After five minutes, there was a steady increase in the counts but it began to fall after the 20-minute mark.

\subsubsection{Average $L_{o}$ and $L_{d}$ domain size}

\subsubsection{Low fengycin doses}

In the $0 \%, 3 \%$, and $6 \%$ ergosterol trials, the $\mathrm{L}_{\mathrm{o}}$ domain size remained consistent throughout the incubation period (Fig. 3.1B). In the $0 \%$ and $6 \%$ ergosterol trials the average size of the domains remained the same (Fig. 3.1B), while there was a smaller average size in the 3\% ergosterol trials. The $12 \%$ ergosterol trials saw an immediate drop in average domain size, which did not return to their original size (Fig. 3.1B). More change was seen amongst the size of $\mathrm{L}_{d}$ domains. In the $0 \%$ ergosterol trials, $L_{d}$ domain sizes remained relatively constant minus a spike in size at the 
second dose application (Fig. 3.2B). Domains returned to their original size within five minutes. Domains in the $3 \%$ ergosterol trail remained relatively consistent, these trials contained the smallest sized domains. The $6 \%$ ergosterol trials were the only ones which saw a large increase in $\mathrm{L}_{\mathrm{d}}$ domain size after the first fengycin dose (Fig. 3.2B). After incubation, domain sizes resembled those at $\mathrm{T}_{0}$. The $12 \%$ ergosterol trials saw a sudden decrease in domain sizes at the second dose application, but returned to their original size at the end of the trial period. While there were fluctuations, the average sizes at the end were very similar to those seen at the beginning of the incubation.

\subsubsection{High fengycin dose}

There was an immediate decrease in the $\mathrm{L}_{\mathrm{o}}$ domain size in the $0 \%$ ergosterol trials, which did not return to their original size over time (Fig. 3.3B). Domains in the $3 \%$ ergosterol trials exhibited a similar trend except for an initial decrease, half that seen in the $0 \%$ ergosterol trials and for an increase in size during the last 20 minutes of the incubation period. Size in the $6 \%$ ergosterol trials was more consistent with an overall gradual decrease in $\mathrm{L}_{\mathrm{o}}$ domain size in the last 20 minutes. The $12 \%$ ergosterol trials saw the largest decrease in average $\mathrm{L}_{\mathrm{o}}$ domain size, approximately twice that seen in the $0 \%$ ergosterol trials, within the first five minutes. By the end of the incubation, the domains had increased in sized and matched that seen in the $0 \%$ ergosterol trials.

A slight decrease in average $\mathrm{L}_{d}$ domain size was seen in the $0 \%$ ergosterol trials (Fig. 3.4B), after incubation with $500 \mu \mathrm{g}$. Many fluctuations were seen in the 3\% ergosterol trials. After the $500 \mu \mathrm{g}$ fengycin dose application, an increase in $\mathrm{L}_{d}$ domain size was seen. Peak size was seen at

five minutes, after which the size immediately decreased. In the last 20 minutes of the incubation 
period, there was a gradual increase back to their original size. The $6 \%$ ergosterol trials also saw an increase in size, a third that seen in the $3 \%$ ergosterol trials, within the first five minutes, followed by a gradual decrease until the end of incubation. The $12 \%$ ergosterol trials followed a similar trend to that seen in the $0 \%$ ergosterol trials, albeit a larger decrease in size (2-fold) within the first five minutes was seen. While there are slight fluctuations in the average $\mathrm{L}_{\mathrm{d}}$ domain size, the final size was smaller than that observed at $\mathrm{T}_{0}$.

\subsubsection{Percent area taken up by $\mathrm{L}_{\mathrm{o}}$ and $\mathrm{L}_{\mathrm{d}}$ domains}

\subsubsection{Low fengycin doses}

All trials exhibited the same overall trend in the percent area covered by $\mathrm{L}_{\mathrm{o}}$ domains, except during the first five minutes of the dose application. Both the $0 \%$ and $6 \%$ ergosterol trials saw an increase in area taken up by $\mathrm{L}_{o}$ domains (Fig. 3.1C) within the first two minutes of dose application. Ordered domain coverage decreased after five minutes, but saw an increase in coverage again with the second dose application. The $0 \%$ ergosterol trials saw a 6 -fold increase in coverage while the $6 \%$ ergosterol trials saw a 2 -fold increase. An increase in $\mathrm{L}_{0}$ coverage in the $6 \%$ trails coincided with an increase in the number of small sized domains present (Fig. S1 A and C).

The 3\% and $12 \%$ ergosterol trials saw an immediate decrease in $\mathrm{L}_{\mathrm{o}}$ coverage within the first few minutes of dose application. While the $\mathrm{L}_{\mathrm{o}}$ domain counts were similar for the $0 \%$ and $3 \%$ ergosterol trials, the coverage seen in the 3\% trials was approximately half that seen in the $0 \%$ trials (Fig. 1C). Again, the trend seen in coverage mimicked that of the domain count overall and for the small sized domains (Fig. 3.1A and Fig. S1A). The 12\% trials saw an immediate 2-fold decrease in $\mathrm{L}_{\mathrm{o}}$ coverage, with an immediate recovery to its original state. Like all other treatment 
groups, the $\mathrm{L}_{\mathrm{o}}$ coverage mimicked the domain counts overall and for each respective domain size group (Fig. 3.1A and Figs. S1A, C, D, F, G, and I).

The $0 \%$ and $3 \%$ ergosterol trails exhibited similar trends in the $L_{d}$ coverage as seen in their $\mathrm{L}_{\mathrm{o}}$ coverage (Fig. 3.1C and 3.2C). The $6 \%$ ergosterol trials did not follow the same trend. Disordered domain coverage did not exhibit changes immediately following the first dose application, like all other trials. A five-fold increase in coverage was seen after 20 minutes, but coverage had returned to its initial state by the second dose point. Like all other treatment groups, an increase in $L_{d}$ coverage was seen after the second dose application. The $6 \%$ ergosterol trials exhibited the smallest increase in $\mathrm{L}_{d}$ coverage at the second dose point. This increase in coverage was approximately a sixth of that seen in the $0 \%$ trials, half of that seen in the $3 \%$ trials, and a third of that seen in the $12 \%$ trials (Fig. 3.2C). The $12 \%$ ergosterol trials followed a similar trend to that seen in the $0 \%$ and $3 \%$ ergosterol trials. For all trials, the $L_{d}$ coverage mimicked the trends seen in domain count (Fig. 3.2A), specifically the small sized domain count (Figs. S2A).

Overall, all treatment groups saw an increase in $\mathrm{L}_{\mathrm{d}}$ area coverage. After the second fengycin dose, the $12 \%$ ergosterol bilayer exhibited the least amount of change in the total area taken up by $\mathrm{L}_{d}$ domains (Fig. 3.2C). The same can be said for $\mathrm{L}_{o}$ domains, but with a decrease in coverage seen amongst the $12 \%$ ergosterol trials.

\subsubsection{High fengycin dose}

For trials containing $0 \%$ and $6 \%$ ergosterol, the area taken up by $\mathrm{L}_{\mathrm{o}}$ and $\mathrm{L}_{\mathrm{d}}$ domains followed the same trends seen in their respective domain counts (Figs. 3.3A and C; Figs. 3.4A and C). The $\mathrm{L}_{\mathrm{o}}$ coverage trend in the $3 \%$ ergosterol trials was a mirror opposite to its domain counts (Fig. 3.3A and C), while the $\mathrm{L}_{d}$ coverage followed the same trend as its respective counts (Fig. 3.4A and C). 
While the $0 \%$ ergosterol trials did not see an overall increase in the $\mathrm{L}_{o}$ domain coverage, all other treatment groups saw an increase in $\mathrm{L}_{0}$ coverage. Coverage increased as ergosterol content increased. The $12 \%$ ergosterol trails were the only ones which showed a significant increase in $\mathrm{L}_{\mathrm{o}}$ coverage (Fig. 3.3C). This increase in coverage was attributed to an increase in small and medium sized domains (Fig. S3C and F). There was an immediate decrease in the area taken up by $\mathrm{L}_{d}$ domains (Fig. 3.4C), experiencing the opposite trend seen in the domain counts. There was an initial drop in area coverage, and while there were fluctuations, the area taken up by $\mathrm{L}_{\mathrm{d}}$ domains was less than that seen at $\mathrm{T}_{0}$.

\subsubsection{General effect of fengycin on membrane characteristics}

Looking at the $\mathrm{T}_{0}$ TIRF images, all bilayer types exhibited distinct domain separation (Fig. 3.5A, E, I and M). When exposed to the high fengycin dose, the bilayers containing $0 \%$ and $3 \%$ ergosterol lose the appearance of distinct domains, with no separate ordered domains at the end of the incubation period (Fig. 3.5D and H). Bilayers containing higher amounts of ergosterol, $6 \%$ and $12 \%$, exhibited the reemergence of ordered domains by the end of the incubation period (Fig. 3.5L and P). At the lower fengycin dose, the $0 \%$ and $3 \%$ ergosterol bilayers saw a reappearance of ordered domains after the first 40 mins, but lost them with the application of a second fengycin dose (data not shown). The bilayers containing 6\% and 12\% ergosterol displayed similar distributions of domains when exposed both to the low and high doses of fengycin. 
Figure 3.5. Effect of $500 \mu \mathrm{g}$ of fengycin on lipid bilayers. Bilayers contain 3:1 DOPC:DPPC with 0 (AD), 3 (E-H), 6 (I-L), and 12\% (M-P) ergosterol. Fengycin added immediately after the 0 minute mark. Bilayers were visualized using a $568 \mathrm{~nm}$ laser and $651 \mathrm{~nm}$ cube on a Total Internal Reflection Fluorescence (TIRF) microscope. Scale bar is $10 \mathrm{um}$. 


\subsection{Discussion}

Fengycin has gained attention as a strong antifungal agent, showing potential use as a plant protection agent [25]. The efficacy of the cyclic lipopeptide as an antifungal agent as well as preliminary research on the specific fengycin-membrane interaction(s) have been performed [22]. Specifically, recent work has evaluated the application of fengycin to phospholipid monolayers, bilayers, and liposomes $[24,27]$. While ergosterol has been shown to play an important role in its level of activity, the underlying mechanism of fengycin activity is still unclear.

Preliminary work on the supported lipid bilayers showed no changes in domain distribution after an incubation period of 40-minutes, with only slight changes between the 20 and 40-minute test marks (data not shown). Therefore, all trials were run for 40 minutes after each dose application. This time frame for fengycin activity is similar to that seen applied in the literature. For example, Deleu et al. [24] noted an equilibrium time of approximately 30 minutes in DPPC monolayers when studying fengycin insertion.

In general, for both the distributions and characteristics of $\mathrm{L}_{o}$ and $\mathrm{L}_{\mathrm{d}}$ domains, bilayers absent of ergosterol and those containing low (3\%) ergosterol experienced immediate increases in domain counts after the first $50 \mu \mathrm{g}$ dose application. While both treatment groups revealed a return to their original counts after 40 minutes, counts increased again following the second dose application, to numbers higher than that seen after the first dose. In general, the average sizes of the $\mathrm{L}_{o}$ and $\mathrm{L}_{d}$ remained constant, but there was an increase in area covered with the $\mathrm{L}_{o}$ domains, while $\mathrm{L}_{d}$ domains saw a decrease in coverage. This indicated that fengycin caused the redistribution of ordered and disordered domains. While the lipids within the bilayer were able to compensate and correct for the effects of the first fengycin dose, they were unable to tolerate the second dose, as 
indicated by the reduction in ordered phase domains. Reduction in the ability to form ordered domains in the presence of fengycin has been reported previously $[35,36]$.

Bilayers containing $6 \%$ ergosterol experienced an increase in $\mathrm{L}_{\mathrm{o}}$ domain counts, at the first dose application and again at the second dose application. Although this increase in $\mathrm{L}_{\mathrm{o}}$ domain counts showed a similar pattern to that seen in the 0 and $3 \%$ treatments, the final domain count was only one third the increase of the lower ergosterol containing treatments. Similar results were seen in the $\mathrm{L}_{d}$ domain counts. Both domain types remained relatively the same size throughout. Both $\mathrm{L}_{\mathrm{o}}$ and $\mathrm{L}_{\mathrm{d}}$ domains also showed an increase in area coverage. While both domain types experience a similar increase in domain counts, the $\mathrm{L}_{\mathrm{d}}$ domains exhibited a 2 -fold greater increase in area coverage than that of the $\mathrm{L}_{\mathrm{o}}$ domains. Overall, this indicated that bilayers containing $6 \%$ ergosterol experience some disruption in domain distribution when exposed to fengycin. However, less changes were observed than in $0 \%$ and $3 \%$ ergosterol-containing bilayers.

The $12 \%$ ergosterol bilayers experienced the least amount of change overall at the lower fengycin dose. Domain counts for both the ordered and disordered phases returned to their original numbers after the full incubation period following two $50 \mu \mathrm{g}$ fengycin dose applications. The initial and final coverages of both domain types was approximately equal. The only main difference within this treatment group was the significant decrease in average $\mathrm{L}_{0}$ domain size. While lower ergosterol (primarily $0 \%$ and $3 \%$ ) revealed that changes in membrane characteristics were maintained over the entire incubation period, the $12 \%$ ergosterol bilayer returned to its initial state. This bilayer maintained the presence of distinct ordered domains.

The application of one $500 \mu \mathrm{g}$ dose, in both the bilayers absent of ergosterol and those containing 3\% ergosterol, caused a rapid increase in all parameters of the $\mathrm{L}_{0}$ domains. These increases were observed after approximately the first and fourth minute for bilayers containing $0 \%$ 
and 3\% ergosterol, respectively. While the $\mathrm{L}_{\mathrm{d}}$ domains also exhibited increases in the domain count and percent area, within the first five minutes of exposure, both bilayer models exhibited decreases in the average size of domains.

In bilayers containing $6 \%$ and $12 \%$ ergosterol, a $500 \mu \mathrm{g}$ dose of fengycin revealed an increase in $\mathrm{L}_{\mathrm{o}}$ domain count, but this increase was more pronounced later than with the 0 and $3 \%$ ergosterol bilayers, typically within the five- to 20 -minute marks during the incubation period. While both experienced a decrease in the average $\mathrm{L}_{0}$ domain size, an increase in the area taken up by these domains was seen in the $12 \%$ ergosterol containing bilayers in the last 20 minutes of the incubation period. This was the only bilayer type which showed an increase in area taken up by $\mathrm{L}_{o}$ domains. This could be attributed to the high ergosterol content, which would promote the formation of DPPC-rich domains [37].

Looking at the distribution of $\mathrm{L}_{\mathrm{d}}$ domains, there is an increase in the three parameters within the first five minutes of the $500 \mu \mathrm{g}$ fengycin addition amongst the $6 \%$ ergosterol containing bilayers. Bilayers containing $12 \%$ ergosterol also exhibited an increase in the $\mathrm{L}_{\mathrm{d}}$ domain count but showed gradual decreases in average domain size and area taken up by the domains. Overall, this indicated that bilayers containing higher levels of ergosterol showed an increase in bilayer ordering.

When comparing the $0 \%$ ergosterol bilayers to the $12 \%$ ergosterol bilayers, both the application of low and high doses impacted the characteristics of the domains distributions within the first five minutes of being applied to the $0 \%$ ergosterol containing bilayers. Low doses had little impact on the distribution of domains within the $12 \%$ ergosterol containing bilayers. The high dose of fengycin did impact the $12 \%$ ergosterol containing bilayers, but, unlike the $0 \%$ and $3 \%$ ergosterol containing bilayer, it did not have as great of an impact on domain redistribution. 
Previous work by Deleu et al. [38] studied the effects of fengycin on DPPC lipid monolayers using the Langmuir trough technique in combination with Brewster angle microscopy. They showed a fluidizing effect of fengycin at low molar ratios (0.1-0.5 molar fraction) while at a higher molar ratio (0.66 molar fraction) fengycin dissolved the ordered phase lipid domains. Similarly, our results show at low fengycin exposure while there is redistribution of domains during the incubation period, ordered domains are still present after incubation. At the high fengycin dose, ordered domains were absent at the end of the incubation period in the bilayers containing $0 \%$ and $3 \%$ ergosterol.

TIRF images indicated that all bilayer types revealed a 'smoothing' effect (i.e., a transition from distinct domain separation to a lack of domain separation) after the application of fengycin. Bilayers containing $0 \%$ and $3 \%$ ergosterol maintained the appearance of this smooth effect until the end of the incubation period. Bilayers containing $6 \%$ and $12 \%$ ergosterol reverted to bilayers in which distinct separation of the $\mathrm{L}_{o}$ and $\mathrm{L}_{d}$ domains can be observed. These results suggest that ergosterol plays a role in reducing, and in some cases inhibiting, domain redistribution caused by fengycin or potential readsorption of lipid:fengycin micelles, as has been seen with some surfactins [39], another family of Bacillus cyclic lipopeptides.

In this study, higher concentrations of ergosterol (6 and 12\%) appeared more efficient in buffering the effects of fengycin than lower concentrations ( 0 and 3\%). Work by Fiedler and Heerklotz [27] observed inhibition of liposomal leakage caused by fengycin in POPC vesicles containing $10 \mathrm{~mol} \%$ ergosterol. Overall, this indicates a decrease in fengycin activity with an increased presence of ergosterol.

It is suggested the ergosterol buffers fengycin insertion in the same manner as that of cholesterol. Fiedler and Heerklotz [27] noted $10 \mathrm{~mol} \%$ ergosterol is as effective at inhibiting 
fengycin leakage as $10 \mathrm{~mol} \%$ cholesterol in POPC vesicle leakage. The buffering ability of ergosterol could therefore be attributed to its stiffness, restricting motion within the bilayer [40]. Ergosterol is seen to increase chain order in POPC at concentrations up to $15-20 \mathrm{~mol} \%$ [41, 42].

Deleu et al. [24] found that fengycin inserts itself more deeply into DOPC than DPPC due to the tight packing of DPPC preventing insertion. It is assumed ergosterol acts in a similar fashion to cholesterol, in that it regulates membrane fluidity. Ergosterol contributes to the condensing of unsaturated acyl chains in fluid phases and increases the fluidity of acyl chains in gel/ordered phases such as saturated acyl chains [43]. Published phase diagrams of lipids with cholesterol are plentiful, while comparatively little information is available on phase diagrams that include ergosterol [42, 44-46]. However, the DPPC:ergosterol phase diagram, as determined by Hsueh et al. [45], showed that at room temperature (below the $T_{m}$ of DPPC), the low concentrations of ergosterol used in our study ( 0 and 3\%) would maintain a solid ordered phase ( $\mathrm{S}_{0}$; gel phase) with the DPPC molecules. At 6\% ergosterol, a combination of solid ordered and liquid ordered phases $\left(\mathrm{S}_{\mathrm{o}} / \mathrm{S}_{\mathrm{o}}+\mathrm{L}_{\mathrm{o}}\right)$ would be expected, with more solid ordered phases present. At $12 \%$ ergosterol, full transition to $\mathrm{S}_{\mathrm{o}}+\mathrm{L}_{\mathrm{o}}$ would be attained. This transition is a slight fluidization of the DPPC domains, reducing rigidity yet retaining ordered packing of acyl chains [37, 45].

Other work [41, 42] showed the influence of ergosterol on unsaturated acyl chains using 1palmitoyl-2-oleoyl-sn-glycero-3-phosphocholine (POPC). Introduction of ergosterol to POPC leads to an increase in acyl chain ordering and bilayer, up to concentrations of $25 \mathrm{~mol} \%$ ergosterol. The presence of ergosterol at room temperature leads to the presence of both the ordered and disordered state, two liquid crystalline states [41, 42]. While DOPC differs from POPC, in that it contains two unsaturated acyl chains (18:1) while POPC contains only one (18:1 and 16:0), it may experience similar effects from the presence of ergosterol, similarly to the interactions seen 
between cholesterol and the two phospholipids [47]. Indeed, the addition of cholesterol (0-25 mol\%) to POPC and DOPC membranes led to ordering of the acyl chains, although cholesterol had stronger condensing effects on POPC. This seems to be attributed to POPC containing only one unsaturated acyl chains versus the two unsaturated acyl chains of DOPC [47].

With increasing presence of ergosterol, there would be a decrease in overall membrane fluidity due to the ordering of DOPC. This decrease in fluidity could reduce insertion of fengycin into the bilayer. Hence, a solubilization of bilayers may occur when the bilayer is in a fluid state (e.g., when they lack ergosterol) and may show increased tolerance with a decrease in fluidity and increase in bilayer rigidity (e.g., when they contain higher level of ergosterol). Indeed, TIRF images indicate that bilayers containing $0 \%$ ergosterol were unable to tolerate the insertion of fengycin while bilayers containing $12 \%$ ergosterol remained relatively intact. At low fengycin doses, the acyl chain packing can be seen to influence fengycin activity. With higher concentrations of fengycin, chain ordering is not the only factor to consider; phospholipid charge, acyl chain length, and membrane curvature would influence fengycin's ability to insert itself into the phospholipid bilayer. This effect has been documented in fungal bioassays in which fungi that possessed a higher level of ergosterol in their bilayers were found to be more tolerant to fengycin [25].

Fengycin has been previously stated to act as a heterogeneous perturbant and has a welldefined all-or-none mode of action $[23,24,38,48]$. The presence of ergosterol may be reducing the short-lived interactions of fengycin, at low concentrations, with the membrane. Increasing fengycin concentration may be causing aggregation of the lipopeptide and may be leading to spontaneous curvature of the membrane and disruption of membrane order. Even with the 
incorporation of ergosterol, the bilayer has difficulty tolerating partial or complete insertion of fengycin and withstanding the induced curvature, therefore inducing the membrane to disintegrate.

\subsection{Conclusion}

Ergosterol affects the overall characteristics of the bilayer (fluidity, acyl chain packing, etc.), influencing fengycin's ability to insert itself within the membrane. Bilayers containing higher levels of ergosterol (12\%) showed features (e.g., persistence of ordered domains; Fig. 3.5M and P) characteristic of increased tolerance to activity of fengycin at low doses. Bilayers containing little to no ergosterol $(0 \%$ and $3 \%)$, were more affected by low doses of the antifungal lipopeptide. However, even in the presence of ergosterol, high fengycin doses were able to influence domain distribution within the membrane.

\section{Acknowledgements}

This work was supported by research grant RGPIN-2015-05679 from the Natural Sciences and Engineering Research Council (NSERC) of Canada to T.J.A. 


\subsection{References}

[1] S.J. Singer, G.L. Nicolson, The fluid mosaic model of the structure of cell membranes, Science, 175 (1972) 720-731.

[2] D. Lingwood, K. Simons, Lipid rafts as a membrane-organizing principle, Science, 327 (2010) 46.

[3] J.S. O'Brien, Cell membranes - composition: structure: function, J. Theor. Biol, 15 (1967) 307324.

[4] D.E. Green, A. Tzagoloff, Role of lipids in the structure and function of biological membranes, J. Lipid Res, 7 (1966) 587-602.

[5] F.C. Odds, A.J.P. Brown, N.A.R. Gow, Antifungal agents: mechanisms of action, Trends Microbiol, 11 (2003) 272-279.

[6] T.K. Mazu, B.A. Bricker, H. Flores-Rozas, S.Y. Ablordeppey, The mechanistic targets of antifungal agents: an overview, Mini-Rev. Med. Chem, 16 (2016) 555-578.

[7] S.S. Sardul, S. Harshita, P.A. Ravindra, S. Suneel Kumar and Shyamji, Antifungal azole derivatives and their pharmacological potential: prospects \& retrospects, Nat. Prod. J, 4 (2014) 140-152.

[8] H.V. Bossche, L. Koymans, H. Moereels, P450 inhibitors of use in medical treatment: focus on mechanisms of action, Pharmacol. Ther, 67 (1995) 79-100.

[9] K.C. Gray, D.S. Palacios, I. Dailey, M.M. Endo, B.E. Uno, B.C. Wilcock, M.D. Burke, Amphotericin primarily kills yeast by simply binding ergosterol, Proc. Natl. Acad. Sci, 109 (2012) 2234.

[10] S.M. Hammond, 3 Biological activity of polyene antibiotics, in: G.P. Ellis, G.B. West (Eds.) Progress in Medicinal Chemistry, Elsevier1977, pp. 105-179.

[11] L. Silva, A. Coutinho, A. Fedorov, M. Prieto, Competitive binding of cholesterol and ergosterol to the polyene antibiotic nystatin. A fluorescence study, Biophys. J, 90 (2006) 36253631.

[12] Y.M.t. Welscher, H.H.t. Napel, M.M. Balagué, C.M. Souza, H. Riezman, B. de Kruijff, E. Breukink, Natamycin blocks fungal growth by binding specifically to ergosterol without permeabilizing the membrane, J. Biol. Chem, 283 (2008) 6393-6401. 
[13] Y.M. te Welscher, M.R. van Leeuwen, B. de Kruijff, J. Dijksterhuis, E. Breukink, Polyene antibiotic that inhibits membrane transport proteins, Proc. Natl. Acad. Sci, 109 (2012) 11156.

[14] T.J. Avis, Antifungal compounds that target fungal membranes: applications in plant disease control, Can. J. Plant Pathol, 29 (2007) 323-329.

[15] L.T. Nguyen, E.F. Haney, H.J. Vogel, The expanding scope of antimicrobial peptide structures and their modes of action, Trends Biotechnol, 29 (2011) 464-472.

[16] A.A. Bahar, D. Ren, Antimicrobial peptides, Pharm, 6 (2013).

[17] J. Liu, I. Hagberg, L. Novitsky, H. Hadj-Moussa, T.J. Avis, Interaction of antimicrobial cyclic lipopeptides from Bacillus subtilis influences their effect on spore germination and membrane permeability in fungal plant pathogens, Fungal Biol, 118 (2014) 855-861.

[18] N. Vanittanakom, W. Loeffler, U. Kock, G. Jung, Fengycin-A novel antifungal lipopeptide antibiotic produced by Bacillus subtilis F-29-3, J. Antibiot, 3 (1986) 888-901.

[19] M. Ongena, P. Jacques, Bacillus lipopeptides: versatile weapons for plant disease biocontrol, Trends Microbiol, 16 (2008) 115-125.

[20] L.M. González-Jaramillo, F.J. Aranda, J.A. Teruel, V. Villegas-Escobar, A. Ortiz, Antimycotic activity of fengycin $\mathrm{C}$ biosurfactant and its interaction with phosphatidylcholine model membranes, Colloids Surf. B Biointerfaces, 156 (2017) 114-122.

[21] S. DeFilippi, E. Groulx, M. Megalla, R. Mohamed, T.J. Avis, Fungal competitors affect production of antimicrobial lipopeptides in Bacillus subtilis strain B9-5, J. Chem. Ecol, 44 (2018) 374-383.

[22] J. Falardeau, C. Wise, L. Novitsky, T.J. Avis, Ecological and mechanistic insights into the direct and indirect antimicrobial properties of Bacillus subtilis lipopeptides on plant pathogens, J. Chem. Ecol, 39 (2013) 869-878.

[23] H. Patel, C. Tscheka, K. Edwards, G. Karlsson, H. Heerklotz, All-or-none membrane permeabilization by fengycin-type lipopeptides from Bacillus subtilis QST713, BBA Biomembranes, 1808 (2011) 2000-2008.

[24] M. Deleu, M. Paquot, T. Nylander, Effect of fengycin, a lipopeptide produced by Bacillus subtilis, on model biomembranes, Biophys. J, 94 (2008) 2667-2679.

[25] C. Wise, J. Falardeau, I. Hagberg, T.J. Avis, Cellular lipid composition affects sensitivity of plant pathogens to fengycin, an antifungal compound produced by Bacillus subtilis strain CU12, Phytopathology, 104 (2014) 1036-1041. 
[26] Y. Tao, X.-M. Bie, F.-X. Lv, H.-Z. Zhao, Z.-X. Lu, Antifungal activity and mechanism of fengycin in the presence and absence of commercial surfactin against Rhizopus stolonifer, J. Microbiol, 49 (2011) 146-150.

[27] S. Fiedler, H. Heerklotz, Vesicle leakage reflects the target selectivity of antimicrobial lipopeptides from Bacillus subtilis, Biophys. J, 109 (2015) 2079-2089.

[28] T.J. Avis, R.R. Bélanger, Specificity and mode of action of the antifungal fatty acid cis-9heptadecenoic acid produced by Pseudozyma flocculosa, Appl. Environ. Microbiol, 67 (2001) 956960.

[29] S.J. Attwood, Y. Choi, Z. Leonenko, Preparation of DOPC and DPPC supported planar lipid bilayers for atomic force microscopy and atomic force spectroscopy, Int. J. Mol. Sci, 14 (2013) 3514-3539.

[30] H.M. McConnell, T.H. Watts, R.M. Weis, A.A. Brian, Supported planar membranes in studies of cell-cell recognition in the immune system, Biochim. Biophys. Acta, 864 (1986) 95-106.

[31] L. Kam, S.G. Boxer, Spatially selective manipulation of supported lipid bilayers by laminar flow: steps toward biomembrane microfluidics, Langmuir, 19 (2003) 1624-1631.

[32] E. Mantil, T. Crippin, A. Ianoul, T.J. Avis, Experimental parameters leading to optimal bilayers for total internal reflection fluorescence microscopy visualization, Microsc. Microanal, 23 (2017) 97-112.

[33] E. Akpa, P. Jacques, B. Wathelet, M. Paquot, R. Fuchs, H. Budzikiewicz, P. Thonart, Influence of culture conditions on lipopeptide production by Bacillus subtilis, Appl. Biochem. Biotechnol, 91 551-561.

[34] A.A. Brian, H.M. McConnell, Allogeneic stimulation of cytotoxic T cells by supported planar membranes, Proc. Natl. Acad. Sci, 81 (1984) 6159-6163.

[35] Joshua N. Horn, A. Cravens, A. Grossfield, Interactions between fengycin and model bilayers quantified by coarse-grained molecular dynamics, Biophysical Journal, 105 (2013) 1612-1623.

[36] M. Eeman, M. Deleu, M. Paquot, P. Thonart, Y.F. Dufrêne, Nanoscale Properties of Mixed Fengycin/Ceramide Monolayers Explored Using Atomic Force Microscopy, Langmuir, 21 (2005) 2505-2511.

[37] X. Xu, R. Bittman, G. Duportail, D. Heissler, C. Vilcheze, E. London, Effect of the structure of natural sterols and sphingolipids on the formation of ordered sphingolipid/sterol domains (rafts): 
comparison of cholesterol to plant, fungal, and disease-associated sterols and comparison of sphingomyelin, cerebrosides, and ceramide, J. Biol. Chem, 276 (2001) 33540-33546.

[38] M. Deleu, M. Paquot, T. Nylander, Fengycin interaction with lipid monolayers at the airaqueous interface-implications for the effect of fengycin on biological membranes, J. Colloid Interface Sci, 283 (2005) 358-365.

[39] G. Francius, S. Dufour, M. Deleu, M. Paquot, M.-P. Mingeot-Leclercq, Y.F. Dufrêne, Nanoscale membrane activity of surfactins: influence of geometry, charge and hydrophobicity, BBA - Biomembranes, 1778 (2008) 2058-2068.

[40] D.A. Mannock, R.N.A.H. Lewis, T.P.W. McMullen, R.N. McElhaney, The effect of variations in phospholipid and sterol structure on the nature of lipid-sterol interactions in lipid bilayer model membranes, Chem. Phys. Lipids, 163 (2010) 403-448.

[41] A. Arora, H. Raghuraman, A. Chattopadhyay, Influence of cholesterol and ergosterol on membrane dynamics: a fluorescence approach, Biochem. Biophys. Res. Commun, 318 (2004) 920926.

[42] Y.-W. Hsueh, M.-T. Chen, P.J. Patty, C. Code, J. Cheng, B.J. Frisken, M. Zuckermann, J. Thewalt, Ergosterol in POPC membranes: physical properties and comparison with structurally similar sterols, Biophys. J, 92 (2007) 1606-1615.

[43] D. Marsh, Cholesterol-induced fluid membrane domains: a compendium of lipid-raft ternary phase diagrams, BBA - Biomembranes, 1788 (2009) 2114-2123.

[44] S.L. Veatch, I.V. Polozov, K. Gawrisch, S.L. Keller, Liquid domains in vesicles investigated by NMR and fluorescence microscopy, Biophys. Journal, 86 (2004) 2910-2922.

[45] Y.-W. Hsueh, K. Gilbert, C. Trandum, M. Zuckermann, J. Thewalt, The effect of ergosterol on dipalmitoylphosphatidylcholine bilayers: a deuterium NMR and calorimetric study, Biophys. J, 88 (2005) 1799-1808.

[46] P.F.F. Almeida, Thermodynamics of lipid interactions in complex bilayers, Biochim. Biophys. Acta, 1788 (2009) 72-85.

[47] Brett N. Olsen, Agata A. Bielska, T. Lee, Michael D. Daily, Douglas F. Covey, Paul H. Schlesinger, Nathan A. Baker, Daniel S. Ory, The structural basis of cholesterol accessibility in membranes, Biophys. J, 105 (2013) 1838-1847. 
[48] H. Patel, Q. Huynh, D. Bärlehner, H. Heerklotz, Additive and synergistic membrane permeabilization by antimicrobial (lipo)peptides and detergents, Biophys. J, 106 (2014) 21152125 . 


\title{
Chapter 4
}

Supported lipid bilayers using extracted microbial lipids: domain redistribution in the presence of fengycin

\author{
Elisabeth Mantil ${ }^{\mathrm{a}}$, Trinda Crippina, Tyler J. Avis ${ }^{\mathrm{a}, \mathrm{b},{ }^{*}}$
}

${ }^{1}$ Department of Chemistry, Carleton University. 1125 Colonel By Drive, Ottawa, ON K1S 5B6,

Canada

${ }^{2}$ Institute of Biochemistry, Carleton University, 1125 Colonel By Drive, Ottawa, ON K1S 5B6, Canada 


\begin{abstract}
Fengycin is an antimicrobial cyclic lipopeptide known to interact with microbial cell membranes. In order to gain insight into the role of membranes lipids in fengycin sensitivity, lipids extracted from Alternaria solani, Fusarium sambucinum, and Pythium sulcatum were analyzed and used in the preparation of supported lipid bilayers (SLBs). TR-DHPE was used to evaluate lipid phase separation within the SLBs, and changes in domain distribution with the application of fengycin. A. solani lipid extract contained the highest quantity of ergosterol while $P$. sulcatum contained no ergosterol. The sterol content of the SLBs was strongly correlated with increases in phase separation, suggesting ergosterol may play a role in promoting ordering of the lipid phases in the bilayer. A. solani experienced the least amount of changes in their domain characteristics when exposed to fengycin, suggesting ergosterol may be buffering the effects of the antimicrobial compound. Other factors such as lipid headgroup charge and unsaturation levels may impact fengycin's effects on domain phase separation, but these effects were generally overshadowed by the role of ergosterol. In the absence of ergosterol, in the P. sulcatum bilayers, fengycin caused an increase in lipid phase ordering.
\end{abstract}

Keywords: fengycin, supported lipid bilayers, extracted fungal lipids, ergosterol 


\subsection{Introduction}

Cell membranes are vital in maintaining the integrity of the cell and are a primary structure involved in interactions with the external environment of the cell. These factors have made the cell membrane an attractive target for antimicrobial compounds. More specifically, the increase in fungicide resistance amongst pathogenic molds has spurred interest in the use of such compounds because of their generalized effects on biological membranes [1]. These generalized effects can include activity via pore formation, membrane thinning or influencing the overall charge of the membrane $[1,2]$. One such antimicrobial compound is the lipopeptide fengycin, which is known to inhibit growth of plant pathogenic molds commonly found on crops [3-6].

Fengycin, as well as other biosurfactant lipopeptides such as surfactin and iturin, have been shown to play a key role in the efficacy of biocontrol strains of the bacterium Bacillus subtilis. Research on these three families of lipopeptides produced by $B$. subtilis has focused on the application of the purified metabolites and via application of the bacterium to crops. For example, the efficacy of B. subtilis and its metabolites was shown to control black Sigatoka, caused by Mycosphaerella fijiensis, in bananas [3,4]. Additionally, extracts have proven to be effective in controlling grey mold caused by Botrytis cinerea [7-11] and soft rot caused by Rhizopus stolonifer $[12,13]$. While fengycin has been proven to be an effective biocontrol agent and is described as having an all-or-none mode of action, its mechanisms of activity are not completely understood.

Previous work conducted on fengycin has suggested that the fatty acyl chain of the molecule readily inserts into fluid phased domains, with the cyclic peptide head sitting on top of the membrane [14-17]. At low fengycin concentrations, this action leads to an increase in bilayer thickness. Exposure to high levels of fengycin results in formation of fengycin aggregates causing a positive curvature in the membrane, due to the conical shape of the molecule, introducing pores 
into the membrane and disrupting its barrier capabilities $[14,17]$. Previous research has also suggested that fengycin is a surface-active molecule $[18,19]$ as the membrane's ability to accommodate insertion of fengycin is low [20]. Recent work has also suggested fengycin causes dehydration of the phospholipid heads resulting in a loss of hydrogen bonding capabilities, impairing barrier function of the membrane [19].

The lipid composition of the membrane influences interactions with its environment. While lipids are the main component of fungal membranes, the composition of lipids differs depending on species and their growth conditions [21]. Lipid content of a fungal membrane has been noted to impact the overall efficacy of fengycin [22]. Most notably, the sterol content and ratio of anionic to zwitterionic phospholipids have been reported to influence fengycin activity. Sterols exhibit both condensing and liquefying effects (ordering) of acyl chains of the phospholipids, depending on the physical state of the lipid which overall effects the viscosity and molecular motion of the phospholipid [23]. Some antifungal agents act by interacting with membrane sterols. For example, polyene antimycotics are more effective against fungi with higher quantities of ergosterol and cell without ergosterol are unaffected by these compounds [24-28]. Conversely, fengycin differs from polyenes, in that it is typically less effective against membranes containing higher levels of ergosterol [22, 29].

Recent work has evaluated the application of fengycin to phospholipid monolayers, bilayers, and liposomes composed of pure lipids in binary and ternary mixtures $[14,17,18,29-$ 31]. While such work gives a wealth of information under simplified, well-defined conditions, complementary work involving more complex model systems and, eventually, whole or live cell systems, would be required. In order to gain insight into these more complex interactions, the present work focusses on the use of TIRFM to evaluate changes in lipid domains of SLB's prepared 
using lipids extracted from molds with different sensitivities to fengycin. More precisely, lipids were extracted from Pythium sulcatum, Fusarium sambucinum, and Alternaria solani, which are generally reported as being sensitive, moderately sensitive and tolerant, respectively, to fengycin. Total lipid extracts were used in combination with TR-DHPE $(0.8 \% \mathrm{~mol})$ in the preparation of SLB's, and domain distribution was evaluated after exposure to low and high doses of fengycin. The total lipid extracts were further analyzed to determine the types and quantities of lipids present and to correlate lipid characteristics to fengycin-induced changes in membrane domain distribution.

\subsection{Methods and Materials}

\subsubsection{Microorganisms}

A. solani Sorauer and F. sambucinum Fuckel were obtained from Laboratoire de diagnostic en phytoprotection (MAPAQ, Québec, QC). P. sulcatum R.G. Pratt \& J.E. Mitch was obtained from the Canadian Collection of Fungal Cultures (Agriculture and Agri-Food Canada, Ottawa, ON). Cultures were maintained on potato dextrose agar (PDA, Becton Dickinson, Sparks, MD) at $23^{\circ} \mathrm{C}$.

\subsubsection{Chemicals}

Texas Red® 1,2-dixeadecanoyl-sn-glycero-3-phosphoethanolamine triethylammonium salt (TR-DHPE) was obtained from Life Technologies (Burlington, ON). Reagent grade chloroform $\left(\mathrm{CHCl}_{3}\right)$, potassium hydroxide $(\mathrm{KOH})$, acetone, and perchloric acid were obtained from Caledon Laboratories (Georgetown, ON). BDH Chemicals methanol was obtained from 
VWR (Mississauga, ON). Magnesium sulfate and potassium monophosphate monobasic were obtained from BioShop (Burlington, ON). The SLB buffer used in all bilayer experiments contained sodium chloride $(\mathrm{NaCl})$ from BioShop Canada (Burlington, ON), BioPerformance Certified $(\geq 99.0 \%)$ Trizma ${ }^{\circledR}$ hydrochloride (Tris-HCl) and calcium chloride $\left(\mathrm{CaCl}_{2}\right)$ from Millipore-Sigma (Oakville, ON), and MilliQ water deionized to a resistivity of $18.2 \mathrm{M} \Omega \cdot \mathrm{cm}$. Glass coverslips (\#1.5) from Warner Instruments (Hamden, CT) were conditioned in a boiling Sparkleen (Fisher Scientific, Ottawa, ON) solution for 10 minutes, followed by washing with distilled water for 20 minutes. Coverslips were then dried in a $343^{\circ} \mathrm{C}$ kiln for a minimum of six hours. Fengycin was produced and purified from B. subtilis as described previously [6, 32]. Hexane and acetic acid were obtained from VWR/Anachemia (Lachine, QC). Ammonium molybdate, Fiske and Subbarow reducer, $\mathrm{BF}_{3}$-methanol, ergosterol and the phospholipid standards (L- $\alpha$ -

phosphatidylcholine, 3-sn-phosphatidic acid, L- $\alpha$-phosphatidylethanolamine, 1-(3-snphosphatidyl)-rac-glycerol, L- $\alpha$-phosphatidyl-L-serine, cardiolipin) were purchased from Millipore-Sigma (Oakville, ON). Silica TLC plates (0.5 mm thick) were obtained from Analtech (Newark, DE). Ethyl alcohol (95\%) was obtained from Commercial Alcohols (Brampton, ON).

\subsubsection{Preparation of extracted lipids}

Liquid cultures of $A$. solani and $F$. sambucinum were prepared by inoculating $5 \times 100 \mathrm{ml}$ aliquots of potato dextrose broth (PDB). P. sulcatum was prepared via inoculation of $5 \times 100 \mathrm{ml}$ aliquots of V8 medium ( $200 \mathrm{ml} / \mathrm{L}$ clarified V8 juice and $\left.2 \mathrm{~g} / \mathrm{L} \mathrm{CaCO}_{3}\right)$. All liquid cultures were incubated at $28.5^{\circ} \mathrm{C}$ while being shaken at $190 \mathrm{RPM}$, for 96 hours.

Following incubation, microbial biomass was separated from the culture media by vacuum filtration (Fisher No. 8 filter paper) and frozen $\left(-80^{\circ} \mathrm{C}\right)$ prior to lyophilization. The lyophilized 
biomass was ground to a powder and dissolved in 100:100:50 $\mathrm{ml}$ of chloroform:methanol:water per gram of biomass. Mixtures were agitated (400 RPM) for three hours at room temperature, while being protected from light. Following agitation, mixtures were vacuum filtered and the biomass was re-extracted in 200:100:50 ml chloroform:methanol:water per gram of biomass, and agitated overnight while being protected from light. The solvents were removed via vacuum filtration. Extracts were combined, transferred to separatory funnels and partitioned against chloroform and water. The chloroform phase containing the lipids was collected. Traces of water were removed using anhydrous $\mathrm{MgSO}_{4}$. Lipid solutions were evaporated to dryness using a rotary evaporator. Lipid residues were resuspended in chloroform, layered with nitrogen, and stored at $20^{\circ} \mathrm{C}$ until use for vesicle preparation and lipid analysis.

\subsubsection{Vesicle preparation}

Using aliquots of the extracted lipids, chloroform was evaporated under vacuum until all traces of solvent were absent (minimum of one hour). The lipid film was rehydrated with SLB buffer ( $3 \mathrm{mM} \mathrm{CaCl}_{2}, 10 \mathrm{mM}$ Tris- $\mathrm{HCl}, 100 \mathrm{mM} \mathrm{NaCl} ; \mathrm{pH}$ 7) to a concentration of $0.75 \mathrm{mg}$ extracted lipids $/ \mathrm{ml}$ and left to hydrate for one hour in an $80^{\circ} \mathrm{C}$ water bath while rotating. TR-DHPE was added to all lipid solutions prior to hydration, to a concentration of $0.8 \mathrm{~mol} \%$. Hydrated lipid solutions were sonicated at $80^{\circ} \mathrm{C}$ for one hour (Codyson PS-20 Ultrasonic Cleaner, AC 110-120V, 50-60 Hz). Following sonication, lipid solutions were extruded using gas tight syringes through a $0.5 \mu \mathrm{m}$ polycarbonate membrane at $80^{\circ} \mathrm{C}$, according to manufacturer's instructions (Avanti Polar Lipids). Solutions were brought to room temperature $\left(21^{\circ} \mathrm{C}\right)$ before being stored at $4^{\circ} \mathrm{C}$. Solutions were kept no longer than four days before use. 


\subsubsection{Bilayer formation}

SLBs were prepared via vesicle fusion on conditioned coverslips, as previously described [33-35]. Briefly, $75 \mu \mathrm{L}$ of vesicle solution was aliquoted into a crystallization dish, a conditioned coverslip was placed on top and incubated at $60^{\circ} \mathrm{C}$ for 10 minutes. Following incubation, $5 \mathrm{~cm}$ of Milli-Q water was layered over the coverslip. The coverslip was lifted and shaken to remove excess lipids before being transferred to a slide. The coverslip was anchored to the slide using doublesided tape. Once transferred, the slide was removed from the water layer and dried of excess water.

\subsubsection{Fengycin application}

Initial TIRFM images were taken of each bilayer (as described below) before the addition of fengycin. After obtaining the initial image, $50 \mu \mathrm{g}$ of fengycin was pipetted into the bilayer along the edge of the slide. Following fengycin addition, images were obtained taken immediately and labelled as $T_{0 \mathrm{~min}}$, and sequentially for every minute for the first five minutes $\left(\mathrm{T}_{1 \mathrm{~min}}-\mathrm{T}_{5} \mathrm{~min}\right)$. Images were again taken after $20\left(\mathrm{~T}_{20} \mathrm{~min}\right)$ and 40 minutes $\left(\mathrm{T}_{40} \mathrm{~min}\right)$ of incubation at room temperature. A second $50 \mu \mathrm{g}$ fengycin aliquot was added after the 40 minute incubation and images were taken at the same time periods as the initial fengycin dose. This protocol was also performed on independently prepared bilayers using a single high $(500 \mu \mathrm{g})$ fengycin dose, as well as with the application of Milli-Q water (control). The experiments were performed in quadruplicates. 


\subsubsection{TIRFM imaging}

Imaging of SLBs was completed using an IX83 inverted microscope (Olympus, Richmond Hill, ON), with Z-drift compensation enabled on the cellTIRF-4Line system (Olympus). TRDHPE was excited with the $568 \mathrm{~nm}$ laser and emissions collected using a LF561 cube. Images were collected using a Rolera EM-C ${ }^{2}$ camera (Qimaging, Surrey, BC). MetaMorph ${ }^{\circledR}$ Advanced version 7.8.2.0 software (Molecular Devices, Sunnyvale, CA) and ImageJ were used to analyze images. Total membrane domains absent of fluorescence (lower 25\%) ( $\mathrm{L}_{0}$; ordered) and domains containing above average levels of fluorescence (upper 15\%) $\left(\mathrm{L}_{\mathrm{d}}\right.$; disordered) were outlined and characterized (enumeration, size, and area occupied) as described previously [34]. Images from four independent trials were analyzed for each data point and averaged. After converting the TIRFM image to an 8-bit image, inverting and thresholding, domains were measured using the function Analyze Particles. Briefly, as the image is scanned, domain edges were detected and outlined by the software and when measured was filled to make it colourless. Area was calculated based on the number of pixels counted within each domain (pixel ${ }^{2}$ ) and converted to $\mu \mathrm{m}^{2}$. Percent area was calculated as the number of pixels counted in the highlighted fraction (domains of interest), in comparison to the total pixel count of the image. Images outside the range of circularity were ignored. Circularity was defined as: Circularity $=4 \pi \times\left(\right.$ area/perimeter $\left.{ }^{2}\right)$, where circularity was a set range of 0.00 to 1.00 , with 1.00 indicating a perfect circle and values closer to 0.00 indicates an elongated shape. 


\subsubsection{Typing and quantification of extracted lipids}

\subsubsection{Lipid class separation}

The neutral and polar lipids were separated via acetone precipitation. Acetone $(6 \mathrm{ml})$ was added to the dried lipids, vortexed and stored at $-20^{\circ} \mathrm{C}$ for one hour. Following incubation, samples were centrifuged at $4500 \mathrm{RPM}$ at $4^{\circ} \mathrm{C}$ for 10 minutes. Supernatants were recovered, and the pellets were resuspended in acetone $(1 \mathrm{ml})$, and the process repeated. The supernatants were combined and dried under a stream of nitrogen. This fraction was composed of the neutral lipids. The pellets, containing the polar lipid, were set aside for phospholipid analysis.

\subsubsection{Sterol analysis}

Alkaline hydrolysis of the neutral lipids was carried it by the addition of a 1:10 KOH solution $(30 \% \mathrm{w} / \mathrm{v})$ and ethanol $(95 \%)$. Samples were heated to $95^{\circ} \mathrm{C}$ for 2 hours. Following heating, the samples were partitioned three times against hexane $(5 \mathrm{ml})$. The hexane fractions, containing the unsaponifiable compounds, were combined and dried under a stream of nitrogen. Dried fractions were resuspended in hexane for analysis of ergosterol content. Neutral lipid samples were quantified on an Aligent 5977E Series GC/MSD System with a HP-5MS (5\% Phenyl 95\% dimethylpolysiloxane) capillary column (Agilent, Santa Clara, CA) in split mode. Sterol samples were held at $100^{\circ} \mathrm{C}$ for two minutes followed by a temperature increase of $20^{\circ} \mathrm{C} \min ^{-1}$ to $200^{\circ} \mathrm{C}$, held for one minute, increased to $300^{\circ} \mathrm{C}$ at a rate of $10^{\circ} \mathrm{C} \mathrm{min}^{-1}$, and held for five minutes. 
The injector and detector were set at $250^{\circ} \mathrm{C}$ and $280^{\circ} \mathrm{C}$, respectively. Sterols were quantified with a standard curve of an ergosterol standard.

\subsubsection{Phospholipid analysis}

Polar lipids were reconstituted in chloroform and split in half, with one half of the sample used for quantification of the phospholipid classes and the other for fatty acid analysis. Phospholipids were separated using a preparative silica gel TLC (0.5 mm thick) and a 10:4:2:2:1 chloroform:acetone:methanol:acetic acid:water solvent system. Following migration, iodine vapour exposure was used to locate the phospholipid classes. These classes were identified via comparison of $\mathrm{R}_{\mathrm{f}}$ values to authentic standards. Following evaporation of the iodine, each phospholipid class was scraped from the TLC plate and combined with Bligh-Dyer solution (1:2 $\mathrm{CHCl}_{3}: \mathrm{MeOH}$ ), and vortexed for one minute. Samples were then centrifuged at $4000 \mathrm{RPM}$ for 10 minutes. The supernatants were collected and dried under nitrogen before being stored at $4^{\circ} \mathrm{C}$.

Phospholipid classes were quantified with a colorimetric phosphorus assay, modified from Bartlett [36]. Briefly, one millilitre of perchloric acid was added to each sample and heated to $130^{\circ} \mathrm{C}$ for two hours. After heating, samples were cooled to room temperature on ice. Once cool, distilled water $(7 \mathrm{ml}), 2.5 \% \mathrm{w} / \mathrm{v}$ ammonium molybdate $(1.5 \mathrm{ml})$, and Fiske and Subbarrow reagent $(0.2 \mathrm{ml})$ were added. Samples were placed in boiling water for seven minutes and then allowed to cool for 20 minutes. Absorbance readings were taken at $\lambda 820$ with a $\mathrm{UV}$-visible spectrophotometer (Varian Cary ${ }^{\circledR} 50$ UV-Vis spectrophotometer). Potassium phosphate monobasic was used as a standard.

Fatty acid methyl esters (FAMEs) were prepared using $\mathrm{BF}_{3}$-methanol (14\%) (2 ml per sample) and heated to $70^{\circ} \mathrm{C}$ for one hour. Samples were cooled to room temperature on ice before 
being diluted with water $(2 \mathrm{ml})$ and extracted with hexane $(2 \mathrm{ml})$. The hexane fractions were collected and analyzed. Using the same GC/MSD system described above, the temperature program started at $100^{\circ} \mathrm{C}$ and increased to $200^{\circ} \mathrm{C}$ at a rate of $20^{\circ} \mathrm{C} \mathrm{min}^{-1}$, held for five minutes before increasing to $240^{\circ} \mathrm{C}$, at a rate of $10^{\circ} \mathrm{C}$, holding again for five minutes. Again, the injector and detector were set to $250^{\circ} \mathrm{C}$ and $280^{\circ} \mathrm{C}$, respectively. FAMEs data was acquired using Aligent MassHunter Workstation and MS fragments analyzed, using the 2014 NIST library.

\subsection{Results}

\subsubsection{Domain analysis}

\subsubsection{General domain characteristics}

Distinct separation between the $\mathrm{L}_{\mathrm{o}}$ and $\mathrm{L}_{\mathrm{d}}$ domains was found in the $A$. solani (Fig. 4.1A) and F. sambucinum SLB's (Fig. 1D), whereas P. sulcatum (Fig. 4.1G) exhibited a more even level of fluorescence throughout the bilayer, making it difficult to distinguish separate microdomains. For all three molds, SLB's appeared unchanged following their full incubation period with a low fengycin dose (Fig. 4.1B, E, and H). Conversely, changes in the three SLB's were found following exposure to a high fengycin dose. A. solani (Fig. 4.1C) and F. sambucinum (Fig. 4.1F) began to lose the appearance of distinct separation of domains while P. sulcatum (Fig. 4.1I) began to develop, slightly, this separation of $\mathrm{L}_{o}$ and $\mathrm{L}_{d}$ domains. 
Overall changes in SLBs were similar throughout the incubation period for all molds.

Using the F. sambucinum SLBs as an example, Fig. 4.2 illustrates the changes seen in the SLBs through the incubation period with the high dose of fengycin. Comparing the appearance of the $\mathrm{SLB}$ at $\mathrm{T}_{0 \mathrm{~min}}$ (Fig. 4.2A) and $\mathrm{T}_{1} \min \left(\right.$ Fig. 4.2B), there appears to be an increase in the size of $\mathrm{L}_{\mathrm{d}}$

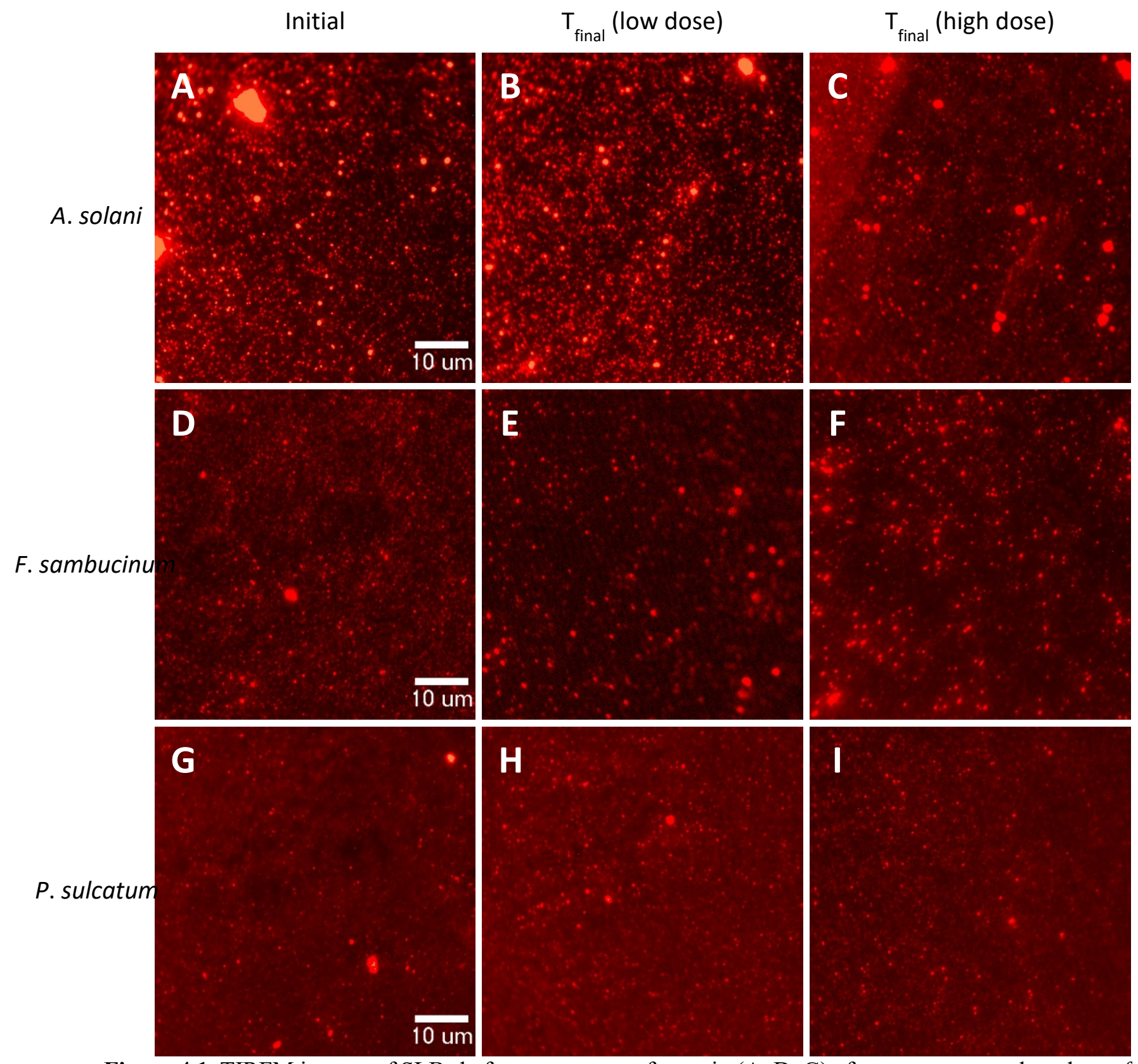

Figure 4.1. TIRFM images of SLBs before exposure to fengycin $(A, D, G)$ after exposure to a low dose of fengycin (two $50 \mu \mathrm{g}$ applications) (B, E, H), and a high dose of fengycin (one $500 \mu \mathrm{g}$ fengycin application) (C, F, I). SLB were prepare from lipids extracts from $A$. solani (A-C), F. sambucinum (D-F), and $P$. sulcatum (G-I). Images visualized with a $568 \mathrm{~nm}$ laser and $561 \mathrm{~nm}$ cube. 
domains and their intensity. By $\mathrm{T}_{5}$ min (Fig. $4.2 \mathrm{C}$ ) the intensity begins to decrease, but the overall prevalence of $L_{d}$ domain appears higher than that seen at $T_{0 \text { min }}$ (Fig. 4.2A). By $T_{20}$ min (Fig. 4.2D) and $\mathrm{T}_{40}$ min (Fig. 4.2E), the intensity of the $\mathrm{L}_{\mathrm{d}}$ domains continues to decrease and the distinct separation between $L_{o}$ and $L_{d}$ phases is less clear. Image analysis was carried out to better identify the $\mathrm{L}_{o}$ and $\mathrm{L}_{d}$ domain distribution trends for SLB from all extracted microbial lipids.
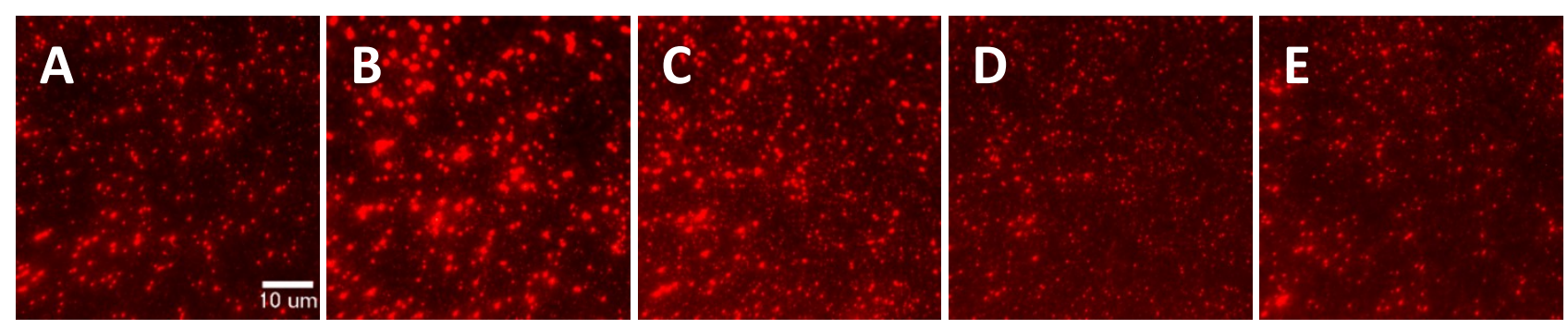

Figure 4.2. TIRFM imaging of $F$. sambucinum SLBs during exposure to $500 \mu \mathrm{g}$ of fengycin, taken at (A) zero, (B) one, (C) five, (D) 20, and (E) 40 minutes. Images visualized using a $568 \mathrm{~nm}$ laser and $561 \mathrm{~nm}$ cube.

\subsubsection{Low fengycin dose}

\subsection{Domain counts}

As seen in Fig. 4.3A, F. sambucinum contained the highest number of ordered domains, approximately twice as many as $P$. sulcatum and 10 -fold more than A. solani. P. sulcatum contained approximately 4 -fold more $\mathrm{L}_{\mathrm{o}}$ domains than A. solani. F. sambucinum bilayers experienced rapid count changes within the first five minutes of fengycin exposure, but remained stable after five minutes, ending at an approximate $25 \%$ overall drop in $\mathrm{L}_{\mathrm{o}}$ counts. Following the second dose, there was an increase in $\mathrm{L}_{0}$ counts at the 41-minute mark after which the counts steadily began to decrease. Ordered domain counts of $P$. sulcatum appeared to be less stable with 
significant fluctuations throughout the incubation period, ultimately ending with a small increase in $\mathrm{L}_{\mathrm{o}}$ domain counts. A. solani $\mathrm{L}_{\mathrm{o}}$ counts remained relatively stable throughout the incubation period. A two-fold increase in counts was recorded after the first dose application, but a return to its original count was seen after the second dose.

F. sambucinum also exhibited the highest number of disordered domains (Fig. 4.4A). P. sulcatum and $A$. solani had counts approximately $50 \%$ lower than $F$. sambucinum. P. sulcatum experienced an increase in the number of $L_{d}$ domains throughout the entire incubation period, ending with an approximate $2 / 3$ increase in counts. F. sambucinum exhibited an increase in counts after two minutes of incubating with the first fengycin dose, but the $L_{d}$ counts fell gradually to approximately $30 \%$ by the second dose point. After the second dose application, an immediate rise in counts were found before again plummeting five minutes following the dose point. The $\mathrm{L}_{\mathrm{d}}$ counts were lowest at the 60-minute mark, but rebounded and, at the end of the incubation period, were approximately $1 / 6$ less than those recorded at $\mathrm{T}_{0 \text { min. }}$. By the end of the incubation period, $F$. sambucinum and P. sulcatum bilayers had similar $\mathrm{L}_{\mathrm{d}}$ domain counts. Similar to its $\mathrm{L}_{\mathrm{o}}$ counts, $A$. solani had relatively stable $\mathrm{L}_{\mathrm{d}}$ counts with a gradual decrease in counts over the entire incubation period, resulting in $1 / 3$ fewer disordered domains. 

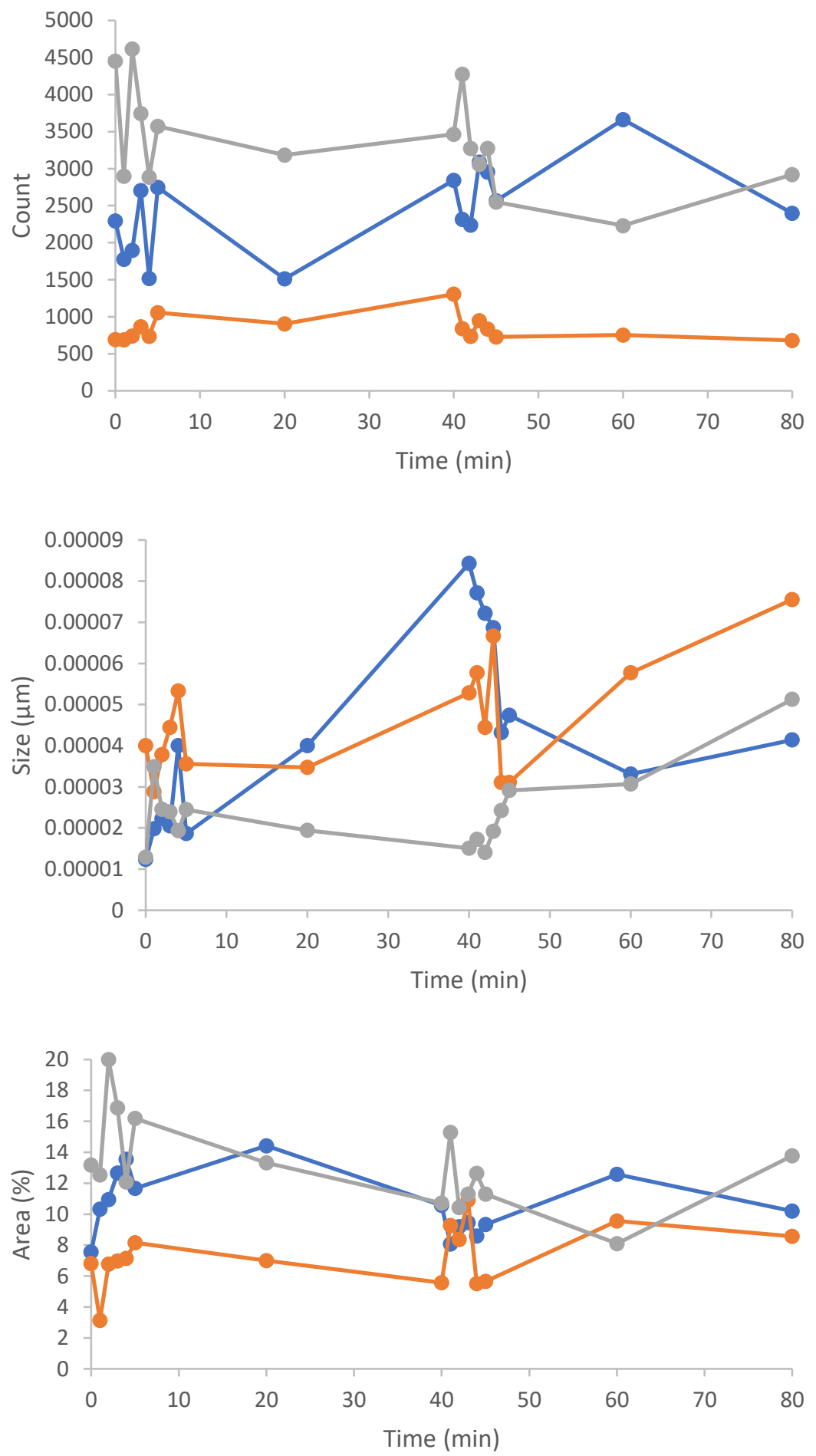

Figure 4.3. Effect of a low dose $(50 \mu \mathrm{g})$ of fengycin on liquid ordered (Lo) domain characteristics and distribution of lipid bilayers. Bilayers contained extracted lipids from Pythium sulcatum (blue), Alternaria solani (orange), and Fusarium sambucinum (grey). Fengycin added at 0 minute (dose 1 ) and the 40 minute (dose 2) marks. (A) $\mathrm{L}_{o}$ domain count; (B) $\mathrm{L}_{o}$ domain size; (C) $\mathrm{L}_{o}$ domain area. 

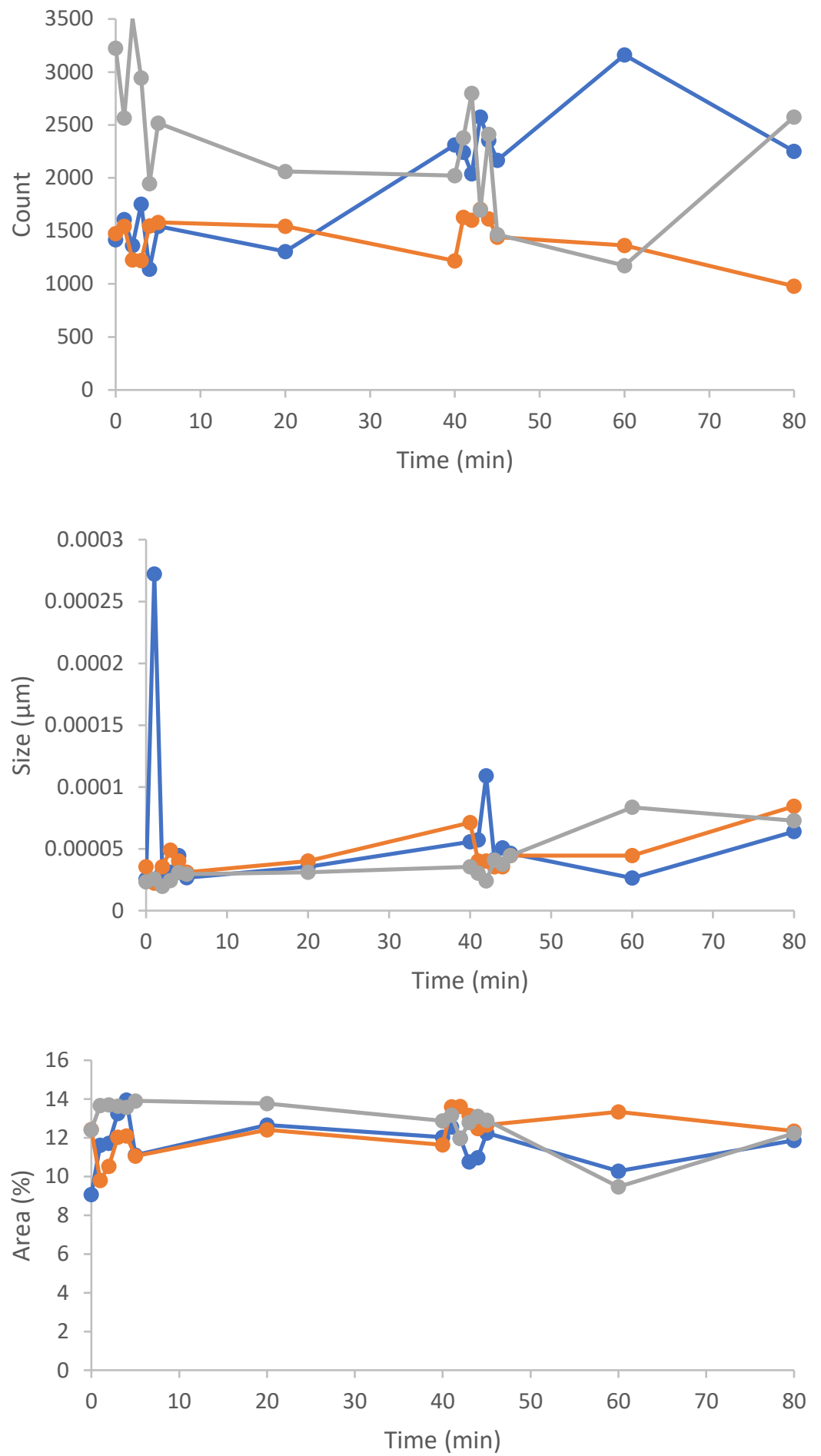

- Pythium - Alternaria - Fusarium

Figure 4.4. Effect of a low dose $(50 \mu \mathrm{g})$ of fengycin on liquid disordered (Ld) domain characteristics and distribution of lipid bilayers. Bilayers contained extracted lipids from Pythium sulcatum (blue), Alternaria solani (orange), and Fusarium sambucinum (grey). Fengycin added at the 0 minute (dose 1) and the 40 minute (dose 2) marks. (A) $\mathrm{L}_{d}$ domain count; (B) $\mathrm{L}_{d}$ domain size; (C) $\mathrm{L}_{d}$ domain area. 


\subsection{Domain size}

P. sulcatum and $F$. sambucinum bilayers began with $\mathrm{L}_{0}$ domains of approximately the same size (Fig. 4.3B). P. sulcatum exhibited a rapid three-fold increase in domain size at the four-minute mark, while $F$. sambucinum experienced a rapid increase at two minutes. P. sulcatum continued to show an increase in $\mathrm{L}_{\mathrm{o}}$ domain size, whereas $F$. sambucinum experienced a decrease. At the second dose, there was a decrease in $P$. sulcatum $\mathrm{L}_{\mathrm{o}}$ domain size, while $F$. sambucinum domain size increased. $P$. sulcatum continued to decrease in ordered domain size minus a small increase in the last 20 minutes, finishing with an average size approximately three-fold large than that at $\mathrm{T}_{0}$ min. Following the second dose application, $F$. sambucinum $\mathrm{L}_{\mathrm{o}}$ domains steadily rose in size, with an overall 2.5 -fold increase in size by the end of the incubation period. A. solani had a $\mathrm{T}_{0} \min$ ordered domain size approximately two-fold larger than the other two fungal SLBs. A. solani experienced similar domain growth to $P$. sulcatum bilayers. Indeed, an increase in size was seen at four minutes followed by a return to their original size before steadily increasing $2 / 3$ in size until the second dose. Following the second dose, there was a spike in average domain size (43-minute mark) before returning to its original size. There was a steady increase in A. solani domain size until the end of the experiment, resulting in a final size approximately twice as large as the $\mathrm{T}_{0} \min$ size.

All fungal bilayers followed approximately the same overall trend for average disordered domain size, an increase in average size. During the first five minutes after fengycin application, all fungal bilayers exhibited a slight increase in $\mathrm{L}_{d}$ domain sizes, which then settled to their original sizes (Fig. 4.4B). An exception to this initial slight increase was $P$. sulcatum, which experienced an approximate 5-fold increase in $\mathrm{L}_{\mathrm{d}}$ domain size after the first minute of fengycin exposure. However, its domains returned to their original size immediately thereafter (two-minute mark). $P$. 
sulcatum disordered domains gradually increased in size until the second dose application, after which the domains doubled in size, gradually decreasing before again increasing in size (50\%). $F$. sambucinum $\mathrm{L}_{\mathrm{d}}$ domain size remained relatively stable during the first 40 minutes of incubation, but gradually increased to approximately twice their original size following the second fengycin dose. A. solani disordered domains doubled in size gradually until the second dose application at which point the sizes decreased and repeated the same trend found in the first 40 minutes of the experiment. By the end of the experiment, A. solani $\mathrm{L}_{\mathrm{d}}$ domains had tripled in size.

\subsection{Domain area}

At the beginning of the experiment, $A$. solani and $P$. sulcatum contained $\mathrm{L}_{\mathrm{o}}$ domains that occupied approximately the same amount of space within their bilayers (Fig. 4.3C). F. sambucinum contained $\mathrm{L}_{\mathrm{o}}$ domains that occupied roughly twice as much area. Overall, $A$. solani and $P$. sulcatum exhibited increase in the area taken up by $\mathrm{L}_{\mathrm{o}}$ domains, whereas $F$. sambucinum was approximately equal at $\mathrm{T}_{0 \mathrm{~min}}$ and $\mathrm{T}_{80 \mathrm{~min}}$. Most changes were found within the first five minutes of fengycin application. All three bilayer types exhibited growth in area covered by $\mathrm{L}_{\mathrm{o}}$ domains, but steadily decreased to the percent area seen at $\mathrm{T}_{0 \text { min. }}$. By the second dosage, both $A$. solani and F. sambucinum again experienced $\mathrm{L}_{\mathrm{o}}$ domain increase in area coverage. $P$. sulcatum exhibited a decrease in $\mathrm{L}_{o}$ area after the second dosage but steadily increased beginning at the 45 -minute mark.

While slight fluctuations were seen, all three fungal bilayers were relatively stable in the area occupied by $L_{d}$ domains (Fig. 4.4C). Both A. solani and F. sambucinum began and ended with the same area coverage. $P$. sulcatum revealed an approximate $2.8 \%$ increase in area covered by disordered domains. 


\subsubsection{High fengycin dose}

\subsection{Domain count}

P. sulcatum and F. sambucinum contained approximately 17 - to 18 -fold more $\mathrm{L}_{\mathrm{o}}$ domains than bilayers composed of $A$. solani lipids (Fig. 4.5A). P. sulcatum and F. sambucinum both saw a gradual increase of approximately $50 \%$ in the number of ordered domains over the full incubation

period. A. solani $\mathrm{L}_{0}$ domains counts remained relatively stable, gradually increasing throughout the incubation period, resulting in an overall doubling of the number of ordered domains present. 

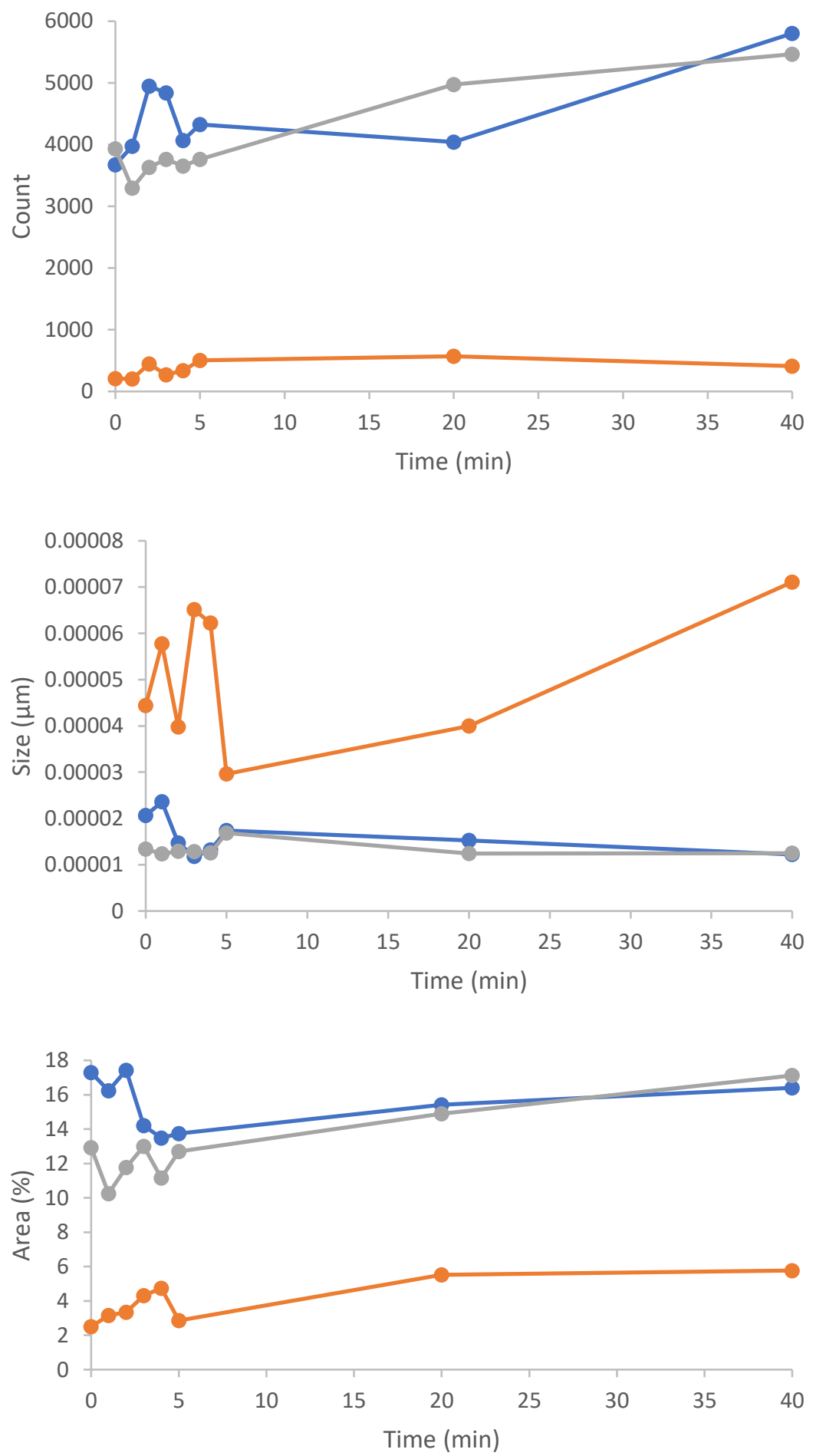

- Pythium $\longrightarrow$ Alternaria - Fusarium

Figure 4.5. Effect of a high dose $(500 \mu \mathrm{g})$ of fengycin on liquid ordered (Lo) domain characteristics and distribution of lipid bilayers. Bilayers contained extracted lipids from Pythium sulcatum (blue), Alternaria solani (orange), and Fusarium sambucinum (grey). Fengycin added at the 0 minute mark. (A) $\mathrm{L}_{\mathrm{o}}$ domain count; (B) $\mathrm{L}_{\mathrm{o}}$ domain size; (C) $\mathrm{L}_{\mathrm{o}}$ domain area. 
P. sulcatum contained the highest number of $\mathrm{L}_{\mathrm{d}}$ domains (Fig. 4.6A). F. sambucinum and A. solani had $1 / 3$ and $1 / 6$ fewer $\mathrm{L}_{\mathrm{d}}$ domains, respectively. P. sulcatum bilayers exhibited growth in the number of ordered domains over the first two to three minutes, settling back to approximately their original counts by four minutes. Following 20 minutes of incubation, a slight decrease in $L_{d}$ domain counts can be seen before again increasing by approximately $1 / 3$ of the $T_{0}$ min count. F. sambucinum exhibited a steady increase in $\mathrm{L}_{\mathrm{d}}$ domain counts throughout the incubation period, ending with approximately $50 \%$ more domains. A. solani $\mathrm{L}_{\mathrm{d}}$ domain counts remained relatively stable with no significant change by the end of the experiment. 

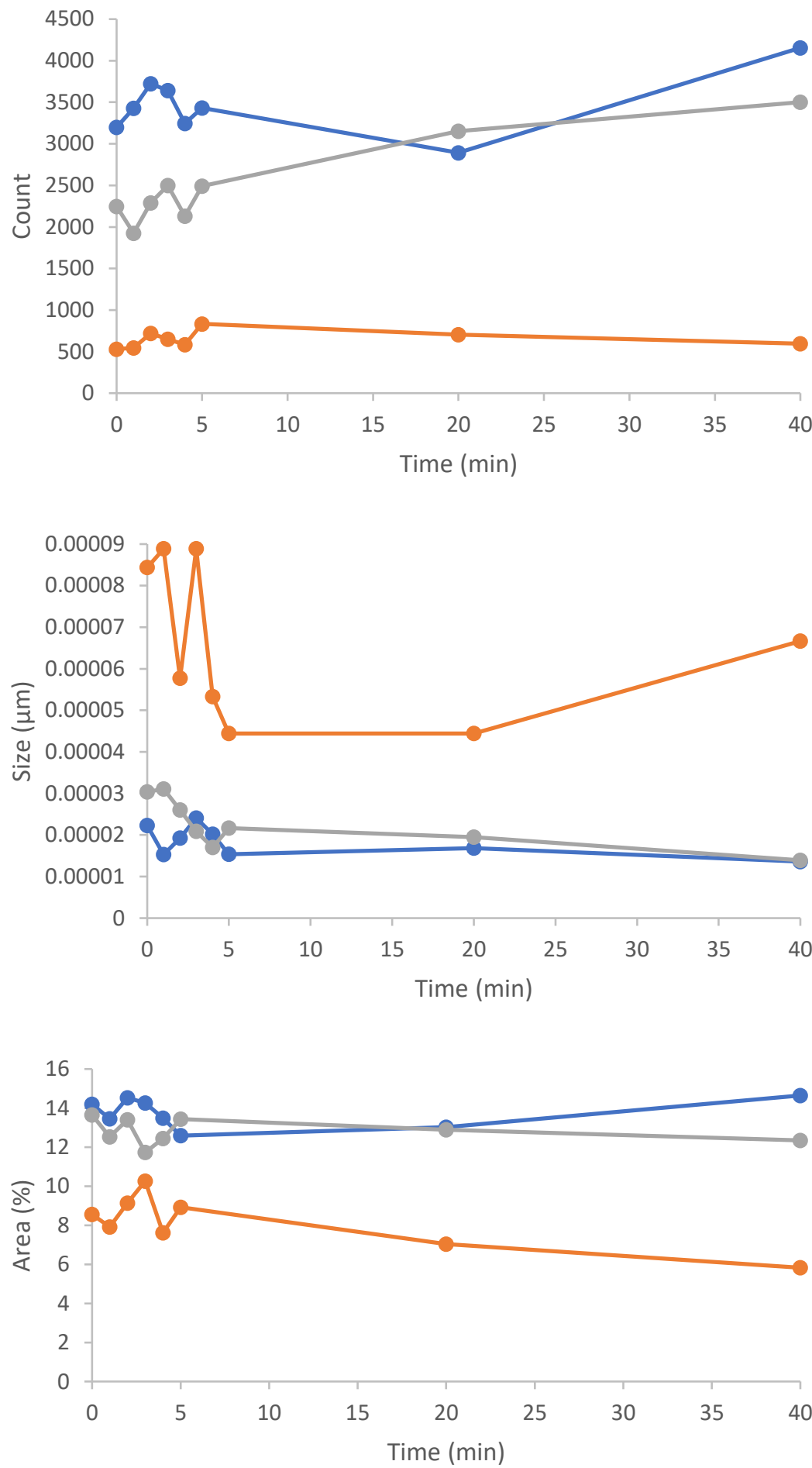

- Pythium - - Alternaria - - Fusarium

Figure 4.6. Effect of a high dose $(500 \mu \mathrm{g})$ of fengycin on liquid disordered (Ld) domain characteristics and distribution of lipid bilayers. Bilayers contained extracted lipids from Pythium sulcatum (blue), Alternaria solani (orange), and Fusarium sambucinum (grey). Fengycin added at the 0 minute mark. (A) $\mathrm{L}_{\mathrm{d}}$ domain count; (B) $\mathrm{L}_{\mathrm{d}}$ domain size; (C) $\mathrm{L}_{\mathrm{d}}$ domain area. 


\subsection{Domain size}

P. sulcatum bilayers contained $\mathrm{L}_{\mathrm{o}}$ domains (Fig. 4.5B) approximately 1.5 -fold larger than F. sambucinum bilayers. A. solani contained the largest sized $\mathrm{L}_{\mathrm{o}}$ domains, just over double the size of $P$. sulcatum domains. Following three minutes of incubation, $P$. sulcatum and $F$. sambucinum contained $\mathrm{L}_{0}$ domains of approximately the same size. Both SLBs exhibited a slight increase in size at five minutes, but gradually decreased over the remainder if the incubation period. $F$. sambucinum contained $\mathrm{L}_{0}$ domains at $\mathrm{T}_{40} \min$ were approximately equal to those at $\mathrm{T}_{0} \min . P$. sulcatum exhibited the same sized $\mathrm{L}_{0}$ domains as those in the $F$. sambucinum $\mathrm{SLBs}$, almost half that of their original size. A. solani SLBs contained $\mathrm{L}_{\mathrm{o}}$ domains that fluctuated drastically throughout the entire incubation period with an approximate $1 / 3$ increase in size by $\mathrm{T}_{5} \mathrm{~min}$. From $\mathrm{T}_{5} \min$ to $\mathrm{T}_{40 \mathrm{~min}}$, a steady increase in domain size occurred, resulting in an overall $2 / 3$ increase in size relative to $\mathrm{T}_{0} \mathrm{~min}$.

A. solani SLBs contained the largest sized $\mathrm{L}_{\mathrm{d}}$ domains (Fig. 4.6B), approximately threeand four-fold larger than those measured in the $F$. sambucinum and $P$. sulcatum SLBs. $F$. sambucinum SLBs contained $\mathrm{L}_{\mathrm{d}}$ domains approximately $1 / 3$ larger than those measured in $P$. sulcatum SLBs. F. sambucinum exhibited a decrease in size during the first five minutes of incubation, resulting in a domain size equal to those seen in the $P$. sulcatum $\mathrm{SLBs}$ at $\mathrm{T}_{0}$ min. A steady decrease in $\mathrm{L}_{\mathrm{d}}$ domains were found until the end of the experiment, resulting in domains approximately half their original size. P. sulcatum SLBs contained $\mathrm{L}_{\mathrm{d}}$ domains that decreased in size by approximately $2 / 3$ during the first five minutes of fengycin exposure and remained steady in size until the end of the experiment. A. solani exhibited a $2 / 3$ reduction in $\mathrm{L}_{\mathrm{d}}$ domain size two minutes after fengycin exposure, quickly returning to their original size by $\mathrm{T}_{3}$ min. At five minutes of exposure, $\mathrm{L}_{\mathrm{d}}$ domains were approximately half their original size and remained that size until 
$\mathrm{T}_{20 \text { min. }}$. During the final 20 minutes of incubation, A. solani SLBs exhibited an increase in their $\mathrm{L}_{\mathrm{d}}$ domain size, ending with domains approximately $20 \%$ smaller than those measured at $\mathrm{T}_{0 \text { min. }}$.

\subsection{Domain area}

As with the other domain characteristics, $P$. sulcatum and $F$. sambucinum followed a very similar trend in $\mathrm{L}_{0}$ domain area coverage from $\mathrm{T}_{5}$ min to $\mathrm{T}_{40 \text { min }}$ (Fig. 4.5C). While there were many fluctuations in area coverage during the first five minutes of fengycin exposure, $F$. sambucinum $\mathrm{L}_{0}$ domains occupied approximately the same area at $\mathrm{T}_{5} \min$ as at the beginning of the experiment. P. sulcatum saw a $3.5 \%$ decrease in $\mathrm{L}_{0}$ area coverage by $\mathrm{T}_{5}$ min. Overall, $F$. sambucinum experienced an approximate $4 \%$ increase in area coverage while $P$. sulcatum bilayers exhibited the same level of $\mathrm{L}_{0}$ domain coverage at $\mathrm{T}_{40} \min$ as at $\mathrm{T}_{0 \text { min. }}$. . solani $\mathrm{SLBs}$ revealed the lowest $\mathrm{L}_{0}$ coverage, which was $10-15 \%$ less than the other fungal bilayers. Overall, there was a steady increase in area occupied by the ordered domains; an approximate doubling in area covered by $\mathrm{L}_{\mathrm{o}}$ domains.

Concerning the area occupied by $\mathrm{L}_{\mathrm{d}}$ domains (Fig. 4.6C), P. sulcatum and $F$. sambucinum again started with a similar level of coverage (approximately 14\%). Both exhibited slight decreases in $\mathrm{L}_{\mathrm{d}}$ coverage at $\mathrm{T}_{1 \text { min, }}$, which immediately rebounded by $\mathrm{T}_{2}$ min. $P$. sulcatum decreased in coverage until $\mathrm{T}_{5} \min$ (approximately $1.6 \%$ less coverage than at $\mathrm{T}_{0} \mathrm{~min}$ ), while $F$. sambucinum exhibited approximately the same level of coverage at $\mathrm{T}_{5} \min$ as at $\mathrm{T}_{0 \mathrm{~min}} . F$. sambucinum SLBs steadily decreased in $\mathrm{L}_{d}$ domain coverage from $\mathrm{T}_{5}$ min to $\mathrm{T}_{40}$ min, ending with approximately $1.3 \%$ less area. $P$. sulcatum revealed the opposite trend, steadily increasing in coverage from $\mathrm{T}_{5} \min$ to $\mathrm{T}_{40} \mathrm{~min}$, ending with approximately half a percent more area taken up by $\mathrm{L}_{\mathrm{d}}$ domains. Disordered domains took up approximately $40 \%$ less area in $A$. solani SLBs and, while there were fluctuations within 
the first five minutes, $T_{5}$ min coverage resembled that of $T_{0 \text { min. }}$. For the remainder of the incubation period, similar to $F$. sambucinum, there was a steady decrease in area taken up by $\mathrm{L}_{\mathrm{d}}$ domains. It saw a decrease 2-fold greater than in the F. sambucinum SLBs.

\subsubsection{Lipid analysis}

\subsubsection{Sterol analysis}

Sterol analysis of the three molds (Table 4.1) showed A. solani contained the highest percentage of ergosterol. F. sambucinum contained 7.5-fold lower ergosterol, while $P$. sulcatum contained no sterols. Correlation analysis (Table 2) showed a strong negative linear correlation between ergosterol content and $\mathrm{L}_{\mathrm{o}}$ domain count, as well as the area occupied by $\mathrm{L}_{\mathrm{o}}$ domains, following exposure to low or high doses of fengycin. A strong positive linear correlation was found between ergosterol content and the average size of $\mathrm{L}_{0}$ domains, following fengycin exposure (low and high doses). Disordered domains also exhibited a strong negative linear correlation between ergosterol content and the number of $\mathrm{L}_{\mathrm{d}}$ domains, while a strong positive linear correlation was seen between ergosterol content and the average size of the $L_{d}$ domains following fengycin exposure (low and high dose). Interestingly, a strong positive linear correlation was seen between ergosterol content and the area occupied by $\mathrm{L}_{\mathrm{d}}$ domains following exposure to a low fengycin dose, whereas exposure to a high fengycin dose resulted in a strong negative linear correlation. 
Table 4.1. Characteristics of lipids extracted from Alternaria solani, Fusarium sambucinum, and Pythium sulcatum.

\begin{tabular}{lccc}
\hline & A. solani & F. sambucinum & P. sulcatum \\
\hline Ergosterol & & & \\
Phospholipid moieties & 3.0 & 0.4 & \\
PA & & & \\
PC & 1.6 & 27.5 & 2.9 \\
PE & 35.9 & 18.9 & 13.8 \\
PG & - & 5.5 & 53.7 \\
PS & 0.5 & 36.8 & 5.5 \\
CL & 35.9 & - & 15.4 \\
Anionic & 1.3 & 11.2 & 8.7 \\
Zwitterionic & 64.1 & 75.5 & 32.5 \\
A:Z & 35.9 & 24.4 & 67.5 \\
& 1.8 & 3.1 & 0.5 \\
Phospholipids fatty & & & \\
acids & & & 47.2 \\
Saturated & 12.6 & 9.8 & 0.0 \\
Monounsaturated & 24.2 & 45.5 & 52.8 \\
Polyunsaturated & 63.2 & 44.6 & 52.8 \\
Total unsaturation & 87.4 & 90.2 & 0.0 \\
M:P & 0.4 & 1.0 & 1.1 \\
U:S & 6.9 & 9.2 & 1.1 \\
$\Delta$ mol total & 1.5 & 1.3 & 18.4 \\
Average acyl chain & 17.8 & 17.8 & \\
length & & & \\
\hline
\end{tabular}


Table 4.2. Correlation of lipid parameters with microbial tolerance to fengycin $(* \mathrm{p}<0.05)$.

\begin{tabular}{|c|c|c|c|c|c|c|}
\hline & $\mathrm{Ld}$ & & & & & \\
\hline $\begin{array}{l}\text { Lipid } \\
\text { parameter }\end{array}$ & Fengycin & $\begin{array}{l}\text { Membrane } \\
\text { characteristic }\end{array}$ & $\begin{array}{l}\text { Correlation } \\
\text { coefficient (r) }\end{array}$ & Fengycin & $\begin{array}{l}\text { Membrane } \\
\text { characteristic }\end{array}$ & $\begin{array}{l}\text { Correlation } \\
\text { coefficient (r) }\end{array}$ \\
\hline \multirow[t]{6}{*}{ Sterol/lipid } & Low & Count & $-0.93 *$ & Low & Count & $-0.94 *$ \\
\hline & & Size & $0.98^{*}$ & & Size & $0.95^{*}$ \\
\hline & & Area & $-0.65^{*}$ & & Area & $0.77 *$ \\
\hline & High & Count & $-0.99 *$ & High & Count & $-0.99 *$ \\
\hline & & Size & $0.99 *$ & & Size & $0.99^{*}$ \\
\hline & & Area & $-0.98^{*}$ & & Area & $-0.99 *$ \\
\hline \multirow[t]{6}{*}{ PE (conical) } & Low & Count & 0.38 & Low & Count & 0.411 \\
\hline & & Size & $-0.78^{*}$ & & Size & $-0.87 *$ \\
\hline & & Area & -0.11 & & Area & $-0.99 *$ \\
\hline & High & Count & $0.62 *$ & High & Count & $0.71^{*}$ \\
\hline & & Size & $-0.58^{*}$ & & Size & $-0.58^{*}$ \\
\hline & & Area & 0.53 & & Area & $0.76^{*}$ \\
\hline \multirow[t]{6}{*}{$\mathrm{A}: \mathrm{Z}$} & Low & Count & 0.22 & Low & Count & 0.19 \\
\hline & & Size & 0.28 & & Size & 0.42 \\
\hline & & Area & $0.67 *$ & & Area & $0.72 *$ \\
\hline & High & Count & -0.05 & High & Count & -0.17 \\
\hline & & Size & 0.01 & & Size & 0.01 \\
\hline & & Area & 0.05 & & Area & -0.25 \\
\hline \multirow[t]{6}{*}{$\mathrm{U}: \mathrm{S}$} & Low & Count & -0.02 & Low & Count & -0.05 \\
\hline & & Size & 0.50 & & Size & $0.63 *$ \\
\hline & & Area & 0.46 & & Area & $0.87 *$ \\
\hline & High & Count & -0.30 & High & Count & -0.41 \\
\hline & & Size & 0.25 & & Size & 0.25 \\
\hline & & Area & -0.19 & & Area & -0.48 \\
\hline \multirow[t]{6}{*}{ M:P } & Low & Count & 0.36 & Low & Count & 0.33 \\
\hline & & Size & 0.13 & & Size & 0.29 \\
\hline & & Area & $0.77 *$ & & Area & $0.62 *$ \\
\hline & High & Count & 0.08 & High & Count & -0.02 \\
\hline & & Size & -0.14 & & Size & -0.13 \\
\hline & & Area & 0.19 & & Area & -0.11 \\
\hline \multirow[t]{6}{*}{$\Delta / \mathrm{mol}$} & Low & Count & $-0.60^{*}$ & Low & Count & $-0.63^{*}$ \\
\hline & & Size & $0.91^{*}$ & & Size & $0.96^{*}$ \\
\hline & & Area & -0.14 & & Area & $0.99^{*}$ \\
\hline & High & Count & $-0.80^{*}$ & High & Count & $-0.86^{*}$ \\
\hline & & Size & $0.77 *$ & & Size & $0.77 *$ \\
\hline & & Area & $-0.73 *$ & & Area & $-0.90^{*}$ \\
\hline \multirow[t]{6}{*}{ Chain length } & Low & Count & 0.32 & Low & Count & 0.35 \\
\hline & & Size & $-0.74^{*}$ & & Size & $-0.83^{*}$ \\
\hline & & Area & -0.17 & & Area & $-0.98 *$ \\
\hline & High & Count & 0.57 & High & Count & $0.66^{*}$ \\
\hline & & Size & -0.52 & & Size & -0.53 \\
\hline & & Area & 0.47 & & Area & $0.72 *$ \\
\hline
\end{tabular}




\subsubsection{Phospholipid moieties}

The polar lipids fractions of the fungal extracts containing the phospholipids were tested for the presence of PA, PC, PE, PG, PS, and CL. F. sambucinum contained a higher amount of PA, PG, and CL compared to the other fungi (Table 4.1). A. solani had higher level of PS and PC, whereas $P$. sulcatum had the highest level of PE. Correlation analysis showed a strong negative linear correlation between PE content and the average size of disordered domains and area occupied by $\mathrm{L}_{d}$ following exposure to both low and high fengycin doses (Table 4.2). A moderate positive linear correlation was found between PE content and the number of $\mathrm{L}_{0}$ domains following exposure to a high fengycin dose. While exhibiting a strong negative linear correlation with ordered domain size after exposure to $500 \mu \mathrm{g}$ of fengycin.

A. solani and F. sambucinum were composed of primarily anionic phospholipid (Table 4.1). Conversely, P. sulcatum was mainly composed of zwitterionic phospholipids. The ratio of anionic to zwitterionic phospholipids (A:Z) exhibited a strong positive correlation to the area occupied up by $\mathrm{L}_{\mathrm{o}}$ and $\mathrm{L}_{\mathrm{d}}$ domains following a low fengycin exposure (Table 4.2).

\subsubsection{Phospholipid FAME analysis}

As shown in Table 1, approximately $90 \%$ of the fatty acids belonging to $A$. solani and $F$. sambucinum were determined to be unsaturated. $P$. sulcatum contained approximately equal amounts of saturated and unsaturated fatty acids. Looking more closely at the ratio of mono- to polyunsaturated fatty acids (M:P), P. sulcatum contained only polyunsaturated fatty acids, $F$. sambucinum contained equal quantities of monounsaturated and polyunsaturated fatty acids. $A$. solani contained approximately 2.5 -fold more polyunsaturated than monounsaturated fatty acids. 
The average degree of unsaturation $(\Delta / \mathrm{mol})$ indicated that $P$. sulcatum was the least unsaturated, whereas A. solani has the highest unsaturation (Table 4.1). P. sulcatum was also determined to contain fatty acids with a longer average acyl chains than the other fungi analyzed.

Following a correlation analysis (Table 4.2), there were few significant correlations between U:S and M:P parameters and membrane domain characteristics. U:S showed a moderate to strong positive linear correlation with $\mathrm{L}_{\mathrm{d}}$ domain size and area at low fengycin doses. M:P showed a moderate to strong positive linear correlation in area for both $\mathrm{L}_{\mathrm{o}}$ and $\mathrm{L}_{\mathrm{d}}$ domains after exposures to low doses of fengycin. The average unsaturation $(\Delta / \mathrm{mol})$ was much more correlated with nearly all membrane domain characteristics. Indeed, a moderate to strong negative linear correlation was found for all counts of $\mathrm{L}_{\mathrm{o}}$ and $\mathrm{L}_{\mathrm{d}}$ domains at both doses of fengycin, as well as area of $\mathrm{L}_{\mathrm{o}}$ and $\mathrm{L}_{\mathrm{d}}$ domains at high fengycin doses. $\Delta / \mathrm{mol}$ also showed a strong positive linear correlation with domain size of $L_{o}$ and $L_{d}$ domains at both doses of fengycin, as well as with area of $L_{d}$ domains with low fengycin doses. Membranes exposed to low levels of fengycin exhibited a strong negative linear correlation between acyl chain length of the fatty acids and both $\mathrm{L}_{\mathrm{o}}$ and $\mathrm{L}_{\mathrm{d}}$ domain sizes. Acyl chain length also exhibited a strong negative linear correlation with the area occupied by disordered domains following low level fengycin exposure. The opposite trend was found amongst membranes following exposure to a high dose of fengycin, where both the $\mathrm{L}_{\mathrm{d}}$ count and area occupied by $\mathrm{L}_{\mathrm{d}}$ domains was positively linearly correlated.

\subsection{Discussion}

While its implication in biological control has been well documented [3-5, 7-13], fengycin's specific mechanism of action is still not clear. It is suggested that fengycin inserts into fluid lipid phases of membranes, with its fatty acyl chain residing within the bilayer and the cyclic 
peptide head sitting on top $[14,15,17]$. Low concentrations of fengycin have been recorded to increase bilayer thickness, while higher concentrations lead to the formation of fengycin aggregates. These aggregates lead to curvature in the membrane, introducing pores and disrupting membrane integrity $[14,17]$. It has therefore been assumed that fengycin is a surface active molecule [18-20].

In efforts to elucidate the mode of action of fengycin, work has been conducted on the interactions of fengycin with phospholipid monolayers and bilayers $[14,17,18,29,30]$. Work to date has focussed on fengycin application to simple binary and ternary lipid models. Lipid composition of biological membranes, specifically the sterol content and ratio of anionic to zwitterionic phospholipids, has been noted to influence the efficacy of fengycin. Most significant is the level of ergosterol found in fungal membranes, where fengycin is reportedly less effective against membranes containing higher levels of ergosterol [22]. The present study used SLB models containing extracted lipids to assess domain distribution in the presence of fengycin.

The most prominent trend found was the strong linear correlations between the sterol content and domain counts, average size and area occupied, by both $\mathrm{L}_{o}$ and $\mathrm{L}_{d}$ domains. This positive correlation between sterol content and domain sizes would be expected as sterols induce phase separation of liquid-ordered and liquid-disordered domains [23]. A. solani contained the highest level of ergosterol (Table 1) and as a result formed SLBs with the largest sized $\mathrm{L}_{o}$ and $\mathrm{L}_{d}$ domains prior to incubation with fengycin, and maintained this trend following fengycin exposure (low and high doses) (Figs. 3B, 4B, 5B, and 6B). A negative correlation was seen between the sterol content and domain count, after exposure to a low dose of fengycin, could be attributed to the combining of smaller domains, resulting in fewer, but larger domains overall. 
By promoting the phase separation of phospholipids in the membrane, sterols may be promoting insolubility of the membrane. Triton, a surfactant, insolubility of membranes has been documented in membranes with closely packed lipids, specifically saturated lipids, and increasing presence of sterols promoting the formation of lipid rafts [37]. 1,2-dipalmitoyl-sn-glycero-3phosphocholine (DPPC) domains have been noted to be resistant to solubilization by the lipopeptide surfactin and redeposition of phospholipid-surfactin micelles [38, 39]. Resistance to solubilization is directly related to the tight packing of the phospholipids, taking on a gel-fluid phase in the bilayer [38]. These traits agree with previous data in which A. solani was determined to be the most tolerant mold in comparison to F. sambucinum and $P$. sulcatum, when exposed to fengycin [22]. P. sulcatum contained no ergosterol (Table 1) and has previously been shown to be highly sensitive to fengycin [22]. In the present work, P. sulcatum contained smaller sized $\mathrm{L}_{\mathrm{o}}$ and $\mathrm{L}_{\mathrm{d}}$ sized domains at a higher frequency, suggesting a more even mixing of microdomains. $F$. sambucinum follows a similar trend. Previous work has shown the addition of fengycin to influence the domain distribution within a bilayer. Ternary SLBs of 1,2-dioleoyl-sn-glycero-3phosphocholine (DOPC) and DPPC with a 12\% ergosterol content showed an increase in $\mathrm{L}_{\mathrm{o}}$ domain area with the application of fengycin [31]. This redistribution of lipid phases could potentially be attributed to the readsorption of fengycin-phospholipid micelles produced via membrane solubilization [14]. A phenomenon noted in DOPC/DPPC bilayers exposed to lipopeptides [38]. The increase in ordered phase could also be attributed to the fengycin promoting phase separation, which has been recently noted in differential scanning calorimetry (DSC) work [19].

Fengycin has been noted, via calorimetry studies, to induce lipid phase ordering at temperatures below $40^{\circ} \mathrm{C}$ in stratum corneum model membranes [30]. Similar to surfactin, 
fengycin is suggested to preferentially insert along the boundaries of phase separations. The high level of disorganization found along lipid phase boundaries makes for an ideal membrane insertion target for lipopeptides [30, 39]. Low concentrations of lipopeptides can produce lipopeptide rich domains within the bilayer, promoting the ordering of phospholipids [19, 40, 41]. Increasing lipopeptide concentration could lead to perturbations at the phase boundaries leading to increase bilayer thickness and potential impairment of membrane barrier function. These perturbations can also promote lipopeptide-phospholipid micelles [17, 38], giving the lipopeptides a detergent-like mechanism.

In a monolayer system with the presence of the lipopeptide surfactin, the inverted cone structure of the surfactin molecule is thought to counterbalance the hexagonal phase formation of DPPE, stabilizing PE membranes $[39,40]$. Fengycin also takes on an inverted-cone shape and is thought to influence membrane structure in the same manner. Therefore, we would expect to a see stabilization of disordered domains in the membranes that contained higher levels of PE. However, the strong negative linear correlation between PE content and the size of disordered domains (Table 2) indicated that fengycin did not stabilize the disordered domains. In related work, liposomal leakage assays showed that 1-palmitoyl-2-oleoyl-sn-glycero-3-phosphoethanolamine (POPE) was effective in reducing the activity of fengycin [29]. Molecular dynamic simulations have noted a high binding affinity between the negatively charged glutamic acid residues in the peptide ring of fengycin, with the positively charged amine group found on PE [42]. The negative membrane curvature induced by PE counteracting the positive curvature induced by fengycin clusters is thought to the mechanism behind this effect. This interaction is highly unfavourable [15]. While POPE is effective at reducing fengycin activity, the other lipids in its environment influence this relationship. In liposomes leakage assays, the addition of POPE to either 1- 
palmitoyl-2-oleoyl-sn-glycero-3-phosphocholine (POPC) or 1-palmitoyl-2-oleoyl-sn-glycero-3phosphoglycerine (POPG) was more effective at tolerating the effects of fengycin than POPC or POPG alone. However, the combination of POPE with POPG was more tolerant to fengycin activity than POPE with POPC [29]. In molecular dynamic simulations, fengycin aggregates appear to be more stable in PC bilayers than in bilayers containing PE and PG [15, 42]. While it was not possible to identify the true implication of PE in our work, the role of PE in modulating SLB domain distribution in the presence of fengycin seems important and should be explored further.

The ratio of anionic and zwitterionic lipids within the membrane has been reported to play a role in the activity of fengycin. Membranes containing higher levels of anionic lipids have shown reduced sensitivity to fengycin, which is thought to be due to the electrostatic repulsion experienced between the anionic phospholipid and the negatively charged fengycin molecule, at physiological $\mathrm{pH}$ [17]. Previous work has shown membranes with a higher quantity of zwitterionic phospholipids are more sensitive to fengycin, due to increased interactions with the negatively charged fengycin molecule with the surface of the bilayer [17, 22, 43]. Increased interactions with the phospholipid surface suggests increased dehydration of the phospholipids polar head group, reducing hydrogen bonding potential of the phospholipid [19]. Limited hydrogen bonding impacts the phospholipids freedom to move, decreasing its efficacy as a barrier [19]. While previous work has illustrated the impact of membrane charge on fengycin efficacy, these effects could not be seen in the lipid phase domain distribution data obtained.

P. sulcatum contained the highest level of saturated fatty acids and the lowest $\Delta / \mathrm{mol}$ (Table 1). The later phospholipid trait correlated strongly with domain characteristics; there was a strong linear negative correlation between the degree of unsaturation and the number of $\mathrm{L}_{0}$ domains 
(Table 2). Fengycin has been shown to induce phase transitions in saturated bilayers composed of DPPC [19], which could explain the increase in P. sulcatum ordered domain size after exposure to fengycin albeit there is not an increase in counts nor area taken up by the ordered domains. The relatively consistent distribution of domain characteristics throughout $A$. solani SLB exposure to fengycin (low and high does) can potentially be attributed to the greater presence of ergosterol in the bilayer. While an increased presence of polyunsaturated phospholipids would indicate a thinner membrane, prone to leakage, it was experimentally determined that unsaturations beginning at the ninth position, such as those found in $A$. solani, had limited effects on membrane thickness [44]. F. sambucinum and P. sulcatum contained slightly lower quantities of polyunsaturated phospholipids (44.6 and 52.8\%, respectively) and like A. solani, the unsaturations begin at the ninth position, limiting the effects of membrane thinning [44]. The presence of polyunsaturated phospholipids did strongly correlate with an increase in the size of $\mathrm{L}_{o}$ and $\mathrm{L}_{d}$ domains, suggested promotion of lipid phase separation. The presence of ergosterol may also counteract the effects of polyunsaturated lipids in the membrane, reducing the potential for membrane leakage caused by a thinner membrane. Promotion of phase separation can also be attributed to ergosterol content [23].

\subsection{Conclusion}

Sterol content in the SLBs was directly correlated to an increase in phase separation and domain sizes, suggested ergosterol may be promoting lipid ordering within the membrane. SLBs composed of lipids extracted from $A$. solani had the highest sterol content and domain distribution remained the most stable after exposure to fengycin. This result supports previous results showing ergosterol content influences membrane interactions with fengycin [22]. Ergosterol may be buffering the activity of fengycin, enabling more fengycin to incorporate into the bilayer before 
micellar action is seen. While other factors such as the presence of unsaturated lipids and lipid head group charge could modulate fengycin activity, their effects were not well distinguished when evaluating the lipid phase separations in the SLBs. This could be due to the overwhelming effects of ergosterol on bilayer organization, altering the effects of the other bilayer factors. The increased presence of ordered domains in P. sulcatum SLBs also suggests fengycin may be promoting the ordering of phospholipids in these membranes. Recent DSC has also suggested this phenomenon [19]. Future work should look at the use of a fluorescent fengycin analog to evaluate its interaction with the phospholipid phases in real time. 


\subsection{References}

[1] L.T. Nguyen, E.F. Haney, H.J. Vogel, The expanding scope of antimicrobial peptide structures and their modes of action, Trends Biotechnol, 29 (2011) 464-472.

[2] A.A. Bahar, D. Ren, Antimicrobial peptides, Pharm, 6 (2013).

[3] J.A. Gutierrez-Monsalve, S. Mosquera, L.M. González-Jaramillo, J.J. Mira, V. VillegasEscobar, Effective control of black Sigatoka disease using a microbial fungicide based on Bacillus subtilis EA-CB0015 culture, Biological Control, 87 (2015) 39-46.

[4] I. Ceballos, S. Mosquera, M. Angulo, J.J. Mira, L.E. Argel, D. Uribe-Velez, M. RomeroTabarez, S. Orduz-Peralta, V. Villegas, Cultivable bacteria populations associated with leaves of banana and plantain plants and their antagonistic activity against Mycosphaerella fijiensis, Microbial Ecol, 64 (2012) 641-653.

[5] A. On, F. Wong, Q. Ko, R.J. Tweddell, H. Antoun, T.J. Avis, Antifungal effects of compost tea microorganisms on tomato pathogens, Biol Cont, 80 (2015) 63-69.

[6] J. Liu, I. Hagberg, L. Novitsky, H. Hadj-Moussa, T.J. Avis, Interaction of antimicrobial cyclic lipopeptides from Bacillus subtilis influences their effect on spore germination and membrane permeability in fungal plant pathogens, Fungal Biol, 118 (2014) 855-861.

[7] Y. Touré, M. Ongena, P. Jacques, A. Guiro, P. Thonart, Role of lipopeptides produced by Bacillus subtilis GA1 in the reduction of grey mould disease caused by Botrytis cinerea on apple, J Appl Microbiol, 96 (2004) 1151-1160.

[8] Walker, Powell, Seddon, Bacillus isolates from the spermosphere of peas and dwarf French beans with antifungal activity against Botrytis cinerea and Pythium species, J Appl Microbiol, 84 (1998) 791-801.

[9] A. Kefi, I.B. Slimene, I. Karkouch, C. Rihouey, S. Azaeiz, M. Bejaoui, R. Belaid, P. Cosette,

T. Jouenne, F. Limam, Characterization of endophytic Bacillus strains from tomato plants (Lycopersicon esculentum) displaying antifungal activity against Botrytis cinerea Pers, World J Microbiol Biotechnol, 31 (2015) 1967-1976.

[10] R. Walker, C.M.J. Ferguson, N.A. Booth, E.J. Allan, The symbiosis of Bacillus subtilis Lforms with Chinese cabbage seedlings inhibits conidial germination of Botrytis cinerea, Lett Appl Microbiol, 34 (2002) 42-45. 
[11] H. Chen, X. Xiao, J. Wang, L. Wu, Z. Zheng, Z. Yu, Antagonistic effects of volatiles generated by Bacillus subtilis on spore germination and hyphal growth of the plant pathogen, Botrytis cinerea, Biotechnol Lett, 30 (2008) 919-923.

[12] Y. Tao, X.-M. Bie, F.-X. Lv, H.-Z. Zhao, Z.-X. Lu, Antifungal activity and mechanism of fengycin in the presence and absence of commercial surfactin against Rhizopus stolonifer, J. Microbiol, 49 (2011) 146-150.

[13] X. Zhou, Z. Lu, F. Lv, H. Zhao, Y. Wang, X. Bie, Antagonistic action of Bacillus subtilis strain fmbj on the postharvest pathogen Rhizopus stolonifer, J Food Sci, 76 (2011) M254-M259. [14] M. Deleu, M. Paquot, T. Nylander, Effect of fengycin, a lipopeptide produced by Bacillus subtilis, on model biomembranes, Biophys J, 94 (2008) 2667-2679.

[15] Joshua N. Horn, A. Cravens, A. Grossfield, Interactions between fengycin and model bilayers quantified by coarse-grained molecular dynamics, Biophys J, 105 (2013) 1612-1623.

[16] J. Falardeau, C. Wise, L. Novitsky, T.J. Avis, Ecological and mechanistic insights into the direct and indirect antimicrobial properties of Bacillus subtilis lipopeptides on plant pathogens, J. Chem. Ecol, 39 (2013) 869-878.

[17] M. Eeman, L. Pegado, Y.F. Dufrêne, M. Paquot, M. Deleu, Influence of environmental conditions on the interfacial organisation of fengycin, a bioactive lipopeptide produced by Bacillus subtilis, J Colloid Interface Sci, 329 (2009) 253-264.

[18] M. Eeman, M. Deleu, M. Paquot, P. Thonart, Y.F. Dufrêne, Nanoscale properties of mixed fengycin/ceramide monolayers explored using Atomic Force Microscopy, Langmuir, 21 (2005) 2505-2511.

[19] L.M. González-Jaramillo, F.J. Aranda, J.A. Teruel, V. Villegas-Escobar, A. Ortiz, Antimycotic activity of fengycin $\mathrm{C}$ biosurfactant and its interaction with phosphatidylcholine model membranes, Colloids Surf. B Biointerfaces, 156 (2017) 114-122.

[20] H. Patel, C. Tscheka, K. Edwards, G. Karlsson, H. Heerklotz, All-or-none membrane permeabilization by fengycin-type lipopeptides from Bacillus subtilis QST713, BBA Biomembranes, 1808 (2011) 2000-2008.

[21] J. Weete, Fungal lipid biochemistry: distribution and metabolism, Springer Science \& Business Media2012. 
[22] C. Wise, J. Falardeau, I. Hagberg, T.J. Avis, Cellular lipid composition affects sensitivity of plant pathogens to fengycin, an antifungal compound produced by Bacillus subtilis strain CU12, Phytopathology, 104 (2014) 1036-1041.

[23] D.A. Mannock, R.N.A.H. Lewis, T.P.W. McMullen, R.N. McElhaney, The effect of variations in phospholipid and sterol structure on the nature of lipid-sterol interactions in lipid bilayer model membranes, Chem. Phys. Lipids, 163 (2010) 403-448.

[24] K.C. Gray, D.S. Palacios, I. Dailey, M.M. Endo, B.E. Uno, B.C. Wilcock, M.D. Burke, Amphotericin primarily kills yeast by simply binding ergosterol, Proc Natl Acad Sci, 109 (2012) 2234.

[25] S.M. Hammond, 3 Biological activity of polyene antibiotics, in: G.P. Ellis, G.B. West (Eds.) Progress in Medicinal Chemistry, Elsevier 1977, pp. 105-179.

[26] L. Silva, A. Coutinho, A. Fedorov, M. Prieto, Competitive binding of cholesterol and ergosterol to the polyene antibiotic nystatin. A fluorescence study, Biophys J, 90 (2006) 36253631.

[27] Y.M. te Welscher, H.H.t. Napel, M.M. Balagué, C.M. Souza, H. Riezman, B. de Kruijff, E. Breukink, Natamycin blocks fungal growth by binding specifically to ergosterol without permeabilizing the membrane, J. Biol. Chem, 283 (2008) 6393-6401.

[28] Y.M. te Welscher, M.R. van Leeuwen, B. de Kruijff, J. Dijksterhuis, E. Breukink, Polyene antibiotic that inhibits membrane transport proteins, Proc Natl Acad Sci, 109 (2012) 11156.

[29] S. Fiedler, H. Heerklotz, Vesicle leakage reflects the target selectivity of antimicrobial lipopeptides from Bacillus subtilis, Biophys J, 109 (2015) 2079-2089.

[30] M. Eeman, G. Olofsson, E. Sparr, M.N. Nasir, T. Nylander, M. Deleu, Interaction of fengycin with stratum corneum mimicking model membranes: A calorimetry study, Colloid Surf B Biointerfaces, 121 (2014) 27-35.

[31] E. Mantil, T. Crippin, T. Avis, Domain redistribution within ergosterol-containing model membranes in the presence of the antimicrobial compound fengycin, BBA- Biomembranes, (submitted) (2018).

[32] E. Akpa, P. Jacques, B. Wathelet, M. Paquot, R. Fuchs, H. Budzikiewicz, P. Thonart, Influence of culture conditions on lipopeptide production by Bacillus subtilis, Appl Biochem Biotechnol, 91 (2001) 551-561. 
[33] H.M. McConnell, T.H. Watts, R.M. Weis, A.A. Brian, Supported planar membranes in studies of cell-cell recognition in the immune system, Biochim Biophys Acta, 864 (1986) 95-106.

[34] E. Mantil, T. Crippin, A. Ianoul, T.J. Avis, Experimental parameters leading to optimal bilayers for total internal reflection fluorescence microscopy visualization, Microsc Microanal, 23 (2017) 97-112.

[35] A.A. Brian, H.M. McConnell, Allogeneic stimulation of cytotoxic T cells by supported planar membranes, Proc Natl Acad Sci, 81 (1984) 6159-6163.

[36] G.R. Bartlett, Phosphorus assay in column chromatography, J Biol Chem, 234 (1959) 466468.

[37] E. London, Insights into lipid raft structure and formation from experiments in model membranes, Curr Opin Struct Biol, 12 (2002) 480-486.

[38] G. Francius, S. Dufour, M. Deleu, M. Paquot, M.-P. Mingeot-Leclercq, Y.F. Dufrêne, Nanoscale membrane activity of surfactins: influence of geometry, charge and hydrophobicity, BBA - Biomembranes, 1778 (2008) 2058-2068.

[39] O. Bouffioux, A. Berquand, M. Eeman, M. Paquot, Y.F. Dufrêne, R. Brasseur, M. Deleu, Molecular organization of surfactin-phospholipid monolayers: Effect of phospholipid chain length and polar head, BBA - Biomembranes, 1768 (2007) 1758-1768.

[40] A. Grau, J.C. Gómez Fernández, F. Peypoux, A. Ortiz, A study on the interactions of surfactin with phospholipid vesicles,BBA - Biomembranes, 1418 (1999) 307-319.

[41] M. Eeman, G. Francius, Y.F. Dufrêne, K. Nott, M. Paquot, M. Deleu, Effect of cholesterol and fatty acids on the molecular interactions of fengycin with stratum corneum mimicking lipid monolayers, Langmuir, 25 (2009) 3029-3039.

[42] S. Sur, T.D. Romo, A. Grossfield, Selectivity and mechanism of fengycin, an antimicrobial lipopeptide, from molecular dynamics, J Phys Chem B, 122 (2018) 2219-2226.

[43] R. Maget-Dana, M. Ptak, Interactions of surfactin with membrane models, Biophysical Journal, 68 (1995) 1937-1943.

[44] W. Rawicz, K.C. Olbrich, T. McIntosh, D. Needham, E. Evans, Effect of chain length and unsaturation on elasticity of lipid bilayers, Biophys J, 79 (2000) 328-339. 
Chapter 5

Conclusion 


\subsection{Conclusion}

This work analyzed lipid phase separation within in SLBs in the presence of the antimicrobial lipopeptide fengycin. While AFM is commonly used to visualize SLBs, relying on the differences in lipid heights to differentiate between the lipid phases, this study used TIRFM to enable real-time visualization of molecular movement of the phospholipids adhering to the solid support. TIRFM can also be used to track and analyze direct interactions of molecules being added to the bilayers, e.g. antimicrobial compounds such as fengycin. The TIRFM technique can be used for both artificial bilayers and intact cells $[1,2]$.

Limited information is available on SLB protocols for TIRFM and their influence on fluorescent lipid probe distribution within SLBs [3, 4]. Initial work assessed the impact of SLB preparation parameters on the distribution of the fluorescent lipid probe TR-DHPE. Preliminary work also evaluated the use of NBD-PE and BODIPY FL-DHPE but, due to rapid photobleaching, only TR-DHPE was thoroughly analyzed. The work determined the influence of sonication and extrusion during bilayers preparation, and the use of chambers versus conditioned coverslips. The concentration of probe used was taken into consideration as well as the ratio of unsaturated to saturated phospholipids (DOPC:DPPC).

TR-DHPE probe was prone to aggregation, making it difficult to properly visualize the ordered and disordered phases within the SLBs. Liposomal solutions pushed through an extruder following sonication reduced the presence of aggregates; liposomal solutions containing $0.8 \%$ mol TR-DHPE provided good visualization of the phase separations with minimal photobleaching. While phase separations were still highly visible with lower quantities of TR-DHPE, the level of photobleaching made it difficult to image the SLBs. Mechanical shearing induced by extrusion is 
thought to be the main determinant in producing even mixing of lipids in liposomes, leading to even mixing in SLBs. In addition to the even mixing of phospholipids, extrusion forces the production of unilamellar vesicles of a specific size range. An extra hour of sonication did not provide more distinct phase separation in SLBs.

Overall, bilayers composed of a 3:1 ratio of DOPC:DPPC in combination with $0.8 \% \mathrm{~mol}$ TR-DHPE, exposed to one hour of sonication and passed through an extruder, consistently produced high quality bilayers with distinct phase separations. These bilayers were optimal for TIRFM analysis.

Using TIRFM optimal bilayers, varying levels of ergosterol were added $(0,3,6$, and $12 \%)$ to evaluate its influence on phase separation in the presence of the antimicrobial compound fengycin. In the absence of fengycin, higher ergosterol content led to an increase in lipid phase separation, as dictated by the increase in $\mathrm{L}_{\mathrm{o}}$ and $\mathrm{L}_{\mathrm{d}}$ domain sizes. Addition of low doses of fengycin led to disruptions in the $\mathrm{L}_{o}$ and $\mathrm{L}_{d}$ domain distributions (increases in numbers) and this was most prominent in SLBs containing little to no ergosterol. More specifically, SLBs with little to no sterols were able to accommodate an initial low dose of fengycin. However, an additional low dose completely disrupted bilayer organization. Moreover, while disruptions in domain distributions still occurred in bilayers containing higher levels of ergosterol, they were better able to accommodate the presence of fengycin and returned to their original domain distribution.

A high fengycin dose was not accommodated by any of the bilayers. Although increasing ergosterol content in a bilayer made it more tolerant to low doses of fengycin, ergosterol was no longer able to compensate for higher dose of fengycin inserting into the bilayer. All bilayers experienced a smoothing effect, but the 6 and 12\% ergosterol bilayers reverted back to or near to 
their original lipid distribution over time. Overall, bilayers containing higher concentrations of ergosterol were more effective at buffering the effects of fengycin.

Following the evaluation of ternary SLB systems, this work investigated SLBs prepared from lipids extracted from plant pathogens that are known to be sensitive or tolerant to the presence of fengycin. Phase separations in these SLBs were evaluated using TIRFM with TR-DHPE. Strong linear correlations between sterol content and domain distribution were noted. Specifically, there was a strong positive linear correlation between ergosterol content and domain size, which suggested sterols induce phase separation in bilayers [5]. The reduction in domain counts could be attributed to the fusion of small domains to form larger domains.

Bilayers made with $A$. solani lipids were more tolerant to domain redistribution following exposure to fengycin. P. sulcatum SLBs, which contained no ergosterol, revealed a redistribution of microdomains. This agrees with previous data obtain in a bioassay, in which $A$. solani was most tolerant in comparison to $F$. sambucinum and P. sulcatum, when exposed to fengycin. Promotion of phase separation in a bilayer, due to the presence of ergosterol, could be contributing to membrane insolubility. This has been noted in surfactant (Triton) insoluble bilayers [6] and ordered domains insoluble to the cyclic lipopeptide surfactin $[7,8]$.

The redistribution of lipid phases could be attributed to the readsorption of fengycinphospholipid micelles produced via membrane solubilization [7, 9]. An increase in ordered phases could also be attributed to fengycin promoting the ordering of phases, as has been previously shown in DSC work [10]. While other factors such as the lipid headgroup charge, level of unsaturation and phospholipid type have been found to play roles in the activity of fengycin, their effects were not as well distinguished with lipid phase separation using TIRFM. However, it would seem as if phospholipid parameters could modulate sensitivity to fengycin. 
Future work should look at the use of a fluorescent fengycin analog to evaluate its interaction with phospholipid phases in real time. A deeper investigation into the influence of phospholipid type, influencing overall membrane charge and thickness, should be evaluated. More specifically, the role of PE in membrane tolerance to fengycin insertion as well as neighbouring lipids, which may have an impact on the PE-fengycin interaction. Because cellular membranes are not only composed of phospholipids, use of more complex models including the incorporation of transmembrane proteins will need to be evaluated. Their influence in membrane fluidity and overall effect on fengycin activity should be taken into consideration. Knowing membrane characteristics that influence tolerance or sensitivity to fengycin could help in the appropriate application of the compound ( or B. subtilis). This work helps to understand which pathogens would be more sensitive, based on their membrane composition.

Overall, results from this work have confirmed that ergosterol plays a key role in the antimicrobial activity of fengycin. The presence of ergosterol in SLB systems promoted tolerance to domain redistribution induced by fengycin. Ergosterol also led to the increased presence of ordered lipid domains, potentially inducing detergent resistance in the bilayer. We were able to visual the direct influences of fengycin on lipid phase distribution within prepared bilayers, witnessing an ordering effect that fengycin has on phospholipids, whether this influences its antimicrobial mode of action needs to be investigated. This work also showed that TIRFM is a powerful tool in the analysis of molecular interactions within lipid bilayers.

While this work supports membrane disruption occurs due to fengycin application, the overall antifungal capabilities of $B$. subtilis could be attributed to the presence of all three lipopeptide families. The combination of different modes of action may contributed to the microorganisms fungicidal capabilities. 


\subsection{References}

[1] J.E. Shaw, A. Slade, C.M. Yip, Simultaneous in situ total internal reflectance fluorescence/atomic force microscopy studies of DPPC/dPOPC microdomains in supported planar lipid bilayers, J Am Chem Soc, 125 (2003) 11838-11839.

[2] J. Oreopoulos, C.M. Yip, Combinatorial microscopy for the study of protein-membrane interactions in supported lipid bilayers: Order parameter measurements by combined polarized TIRFM/AFM, J Struct Biol, 168 (2009) 21-36.

[3] T. Kaasgaard, C. Leidy, J.H. Crowe, O.G. Mouritsen, K. Jørgensen, Temperature-controlled structure and kinetics of ripple phases in one- and two-component supported lipid bilayers, Biophys J, 85 (2003) 350-360.

[4] A.R. Honerkamp-Smith, S. Veatch, S.L. Keller, An introduction to critical points for biophysicists; observations of compositional heterogeneity in lipid membranes, Biochim Biophys Acta, 1788 (2009) 53-63.

[5] D.A. Mannock, R.N.A.H. Lewis, T.P.W. McMullen, R.N. McElhaney, The effect of variations in phospholipid and sterol structure on the nature of lipid-sterol interactions in lipid bilayer model membranes, Chem Phys Lipids, 163 (2010) 403-448.

[6] E. London, Insights into lipid raft structure and formation from experiments in model membranes, Curr Opin Struct Biol, 12 (2002) 480-486.

[7] G. Francius, S. Dufour, M. Deleu, M. Paquot, M.-P. Mingeot-Leclercq, Y.F. Dufrêne, Nanoscale membrane activity of surfactins: influence of geometry, charge and hydrophobicity, BBA - Biomembranes, 1778 (2008) 2058-2068.

[8] O. Bouffioux, A. Berquand, M. Eeman, M. Paquot, Y.F. Dufrêne, R. Brasseur, M. Deleu, Molecular organization of surfactin-phospholipid monolayers: Effect of phospholipid chain length and polar head, BBA - Biomembranes, 1768 (2007) 1758-1768.

[9] M. Deleu, M. Paquot, T. Nylander, Effect of fengycin, a lipopeptide produced by Bacillus subtilis, on model biomembranes, Biophys J, 94 (2008) 2667-2679.

[10] L.M. González-Jaramillo, F.J. Aranda, J.A. Teruel, V. Villegas-Escobar, A. Ortiz, Antimycotic activity of fengycin $\mathrm{C}$ biosurfactant and its interaction with phosphatidylcholine model membranes, Colloids Surf B Biointerfaces, 156 (2017) 114-122. 
Supplementary Materials 

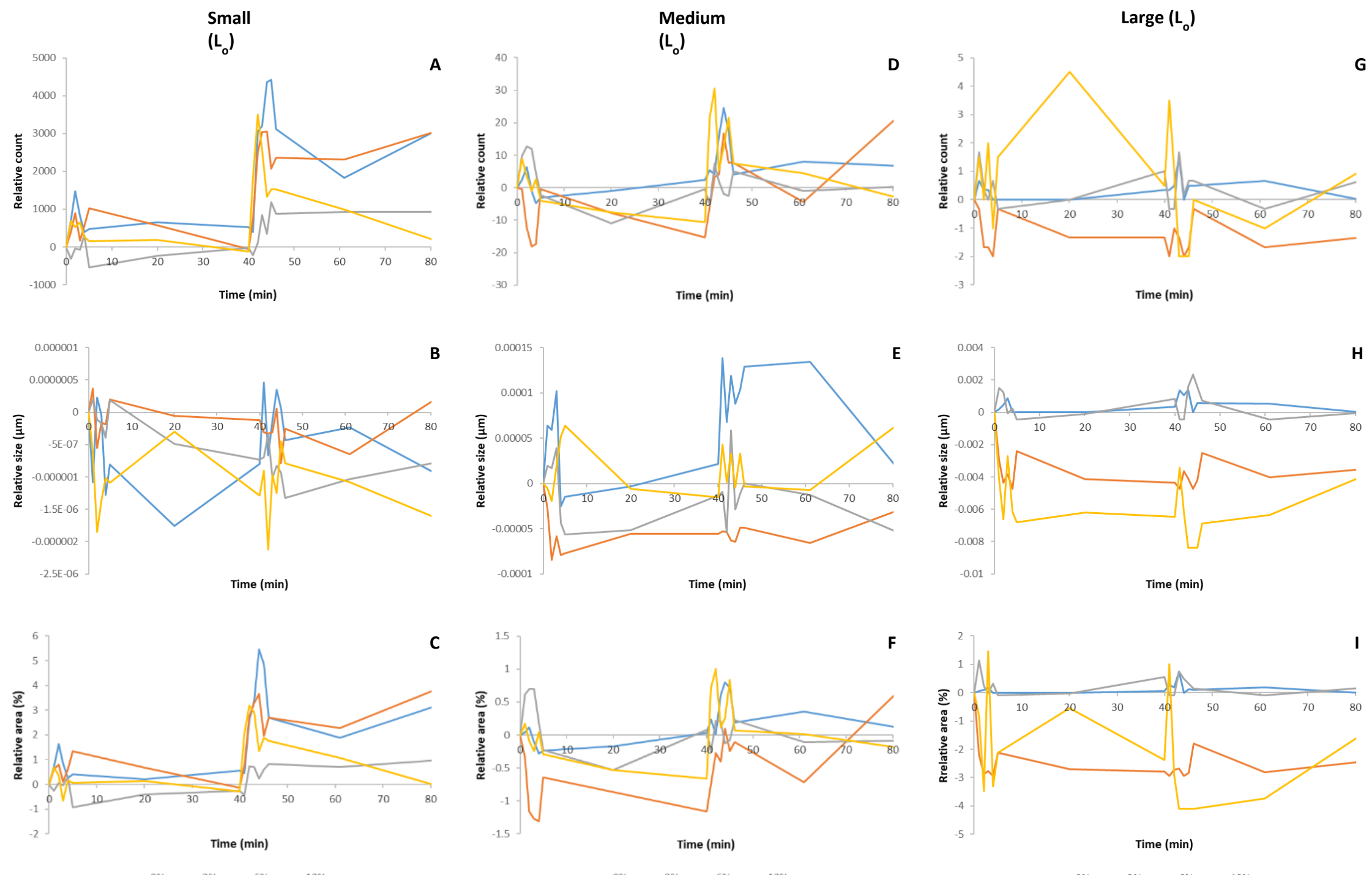

Figure S1. Effect of low doses $(50 \mu \mathrm{g})$ of fengycin on liquid ordered $\left(\mathrm{L}_{\mathrm{o}}\right)$ domain characteristics and distribution of lipid bilayers. Bilayers contained 3:1 DOPC:DPPC with 0 (blue), 3 (orange), 6 (grey), and 12\% (yellow) ergosterol. Fengycin added at the 0 minute (dose 1) and the 40 minute marks (dose 2). A-C. Small domains $(<1 \mu \mathrm{m})$; D-F. Medium domains $(1-10 \mu \mathrm{m})$; G-I. Large domains $(>10 \mu \mathrm{m})$. A, D, G. Relative domain count; B, D, H. Relative domain size; C, F, I. Relative domain area. 

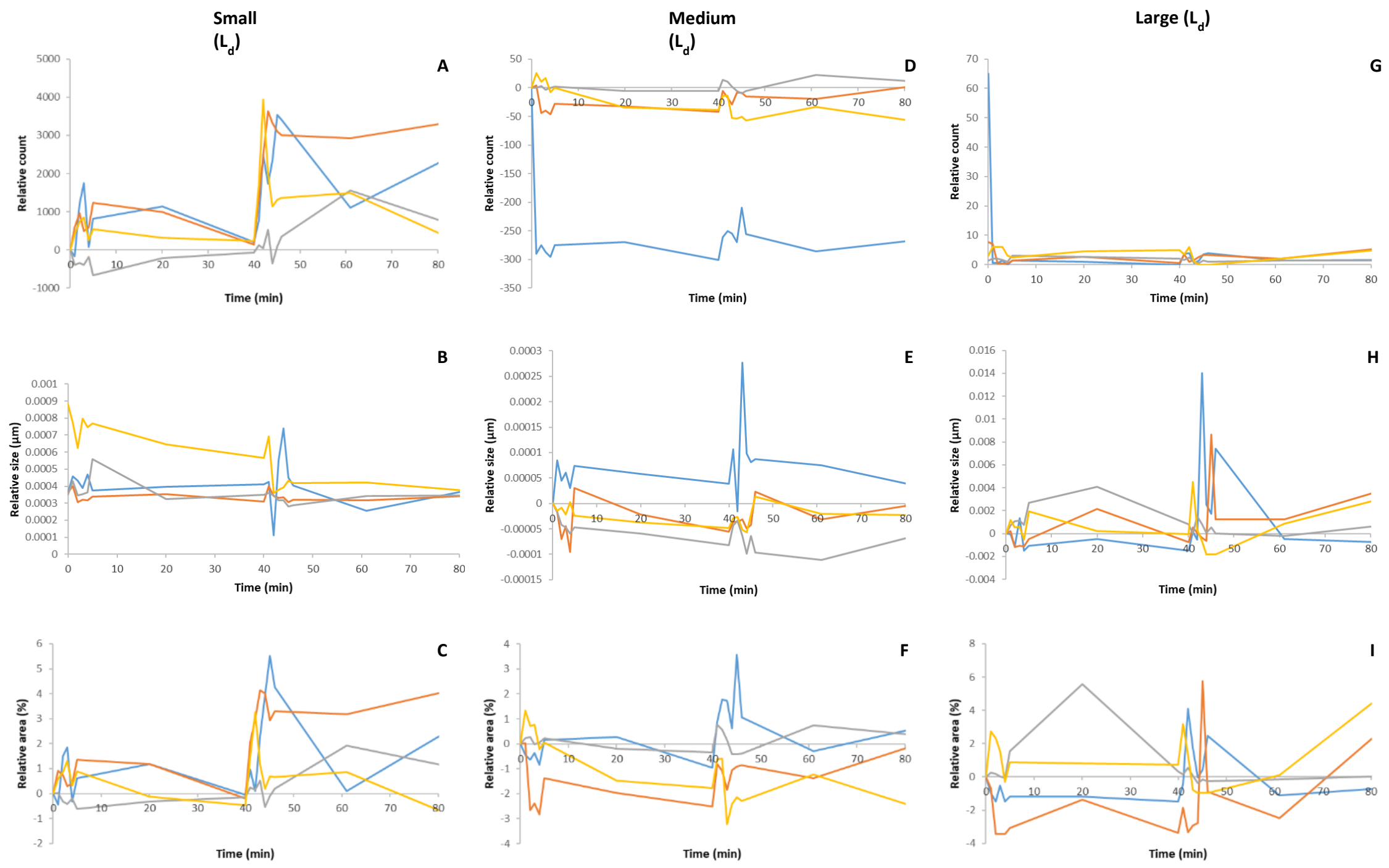

Figure S2. Effect of low doses $(50 \mu \mathrm{g})$ of fengycin on liquid disordered $\left(\mathrm{L}_{\mathrm{d}}\right)$ domain characteristics and distribution of lipid bilayers. Bilayers contained 3:1 DOPC:DPPC with 0 (blue), 3 (orange), 6 (grey), and 12\% (yellow) ergosterol. Fengycin added at the 0 minute (dose 1) and the 40 minute marks (dose 2). A-C. Small domains $(<1 \mu \mathrm{m})$; D-F. Medium domains $(1-10 \mu \mathrm{m})$; G-I. Large domains $(>10 \mu \mathrm{m})$. A, D, G. Relative domain count; B, D, H. Relative domain size; C, F, I. 

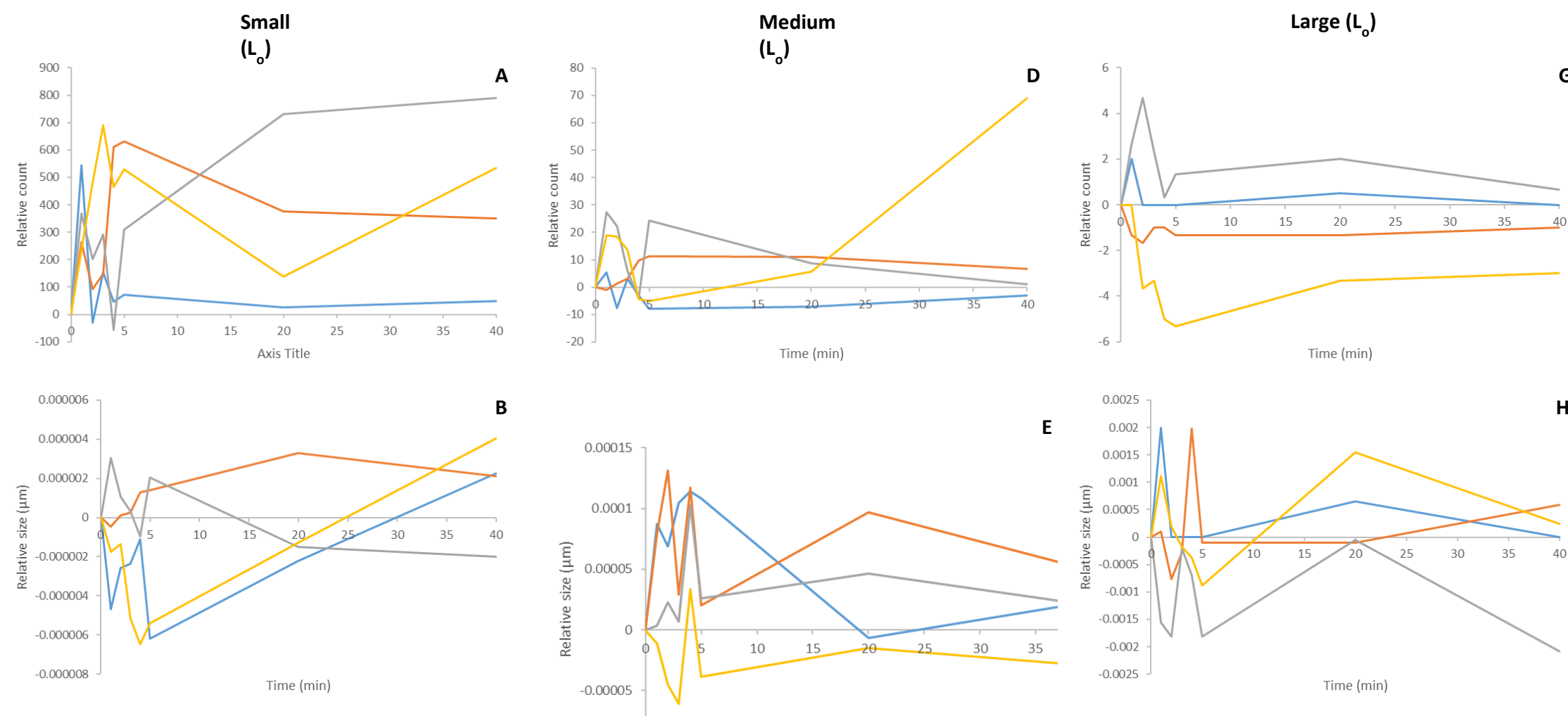

-0.000008 Time $(\mathrm{min})$
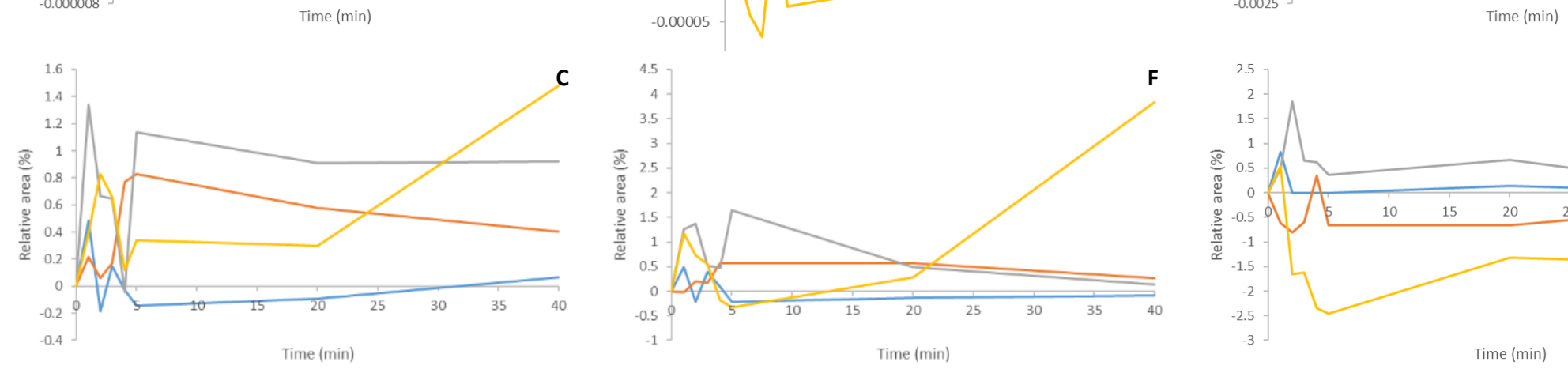

H

$-0 \%-3 \%-6 \%-12 \%$

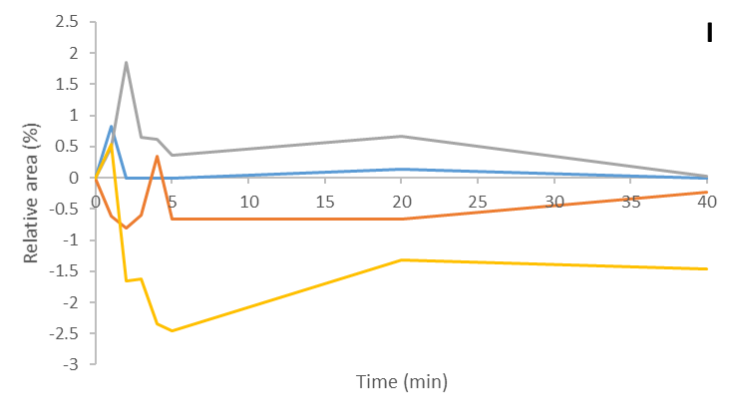

Figure S3. Effect of a high dose $(500 \mu \mathrm{g})$ of fengycin on liquid ordered $\left(\mathrm{L}_{\mathrm{o}}\right)$ domain characteristics and distribution of lipid bilayers. Bilayers contained 3:1 DOPC:DPPC with 0 (blue), 3 (orange), 6 (grey), and 12\% (yellow) ergosterol. Fengycin added at the 0 minute marks. A-C. Small domains (<1 $\mu \mathrm{m})$; D-F. Medium domains (1-10 $\mu \mathrm{m})$; G-I. Large domains (>10 $\mu \mathrm{m})$. A, D, G. Relative domain count; B, D, H. Relative domain size; C, F, I. Relative domain area. 

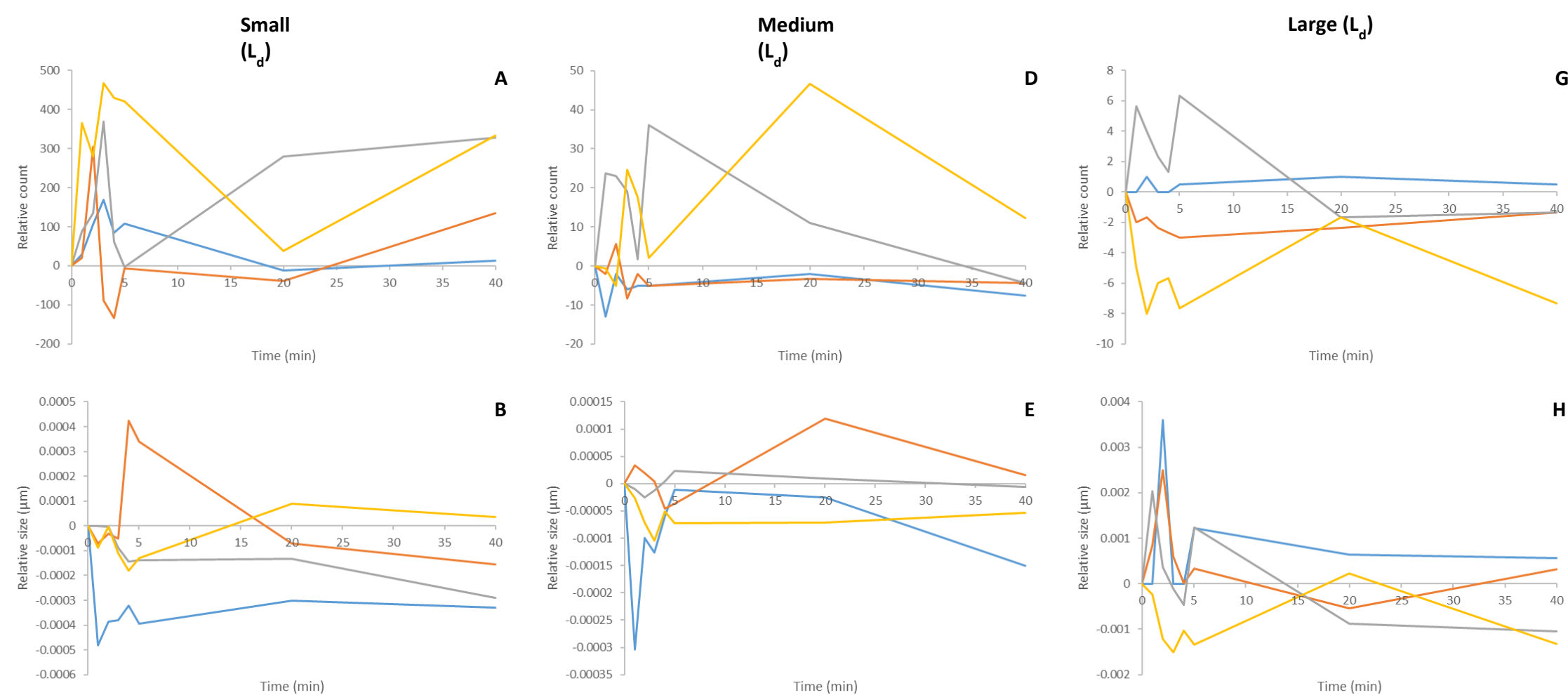

B
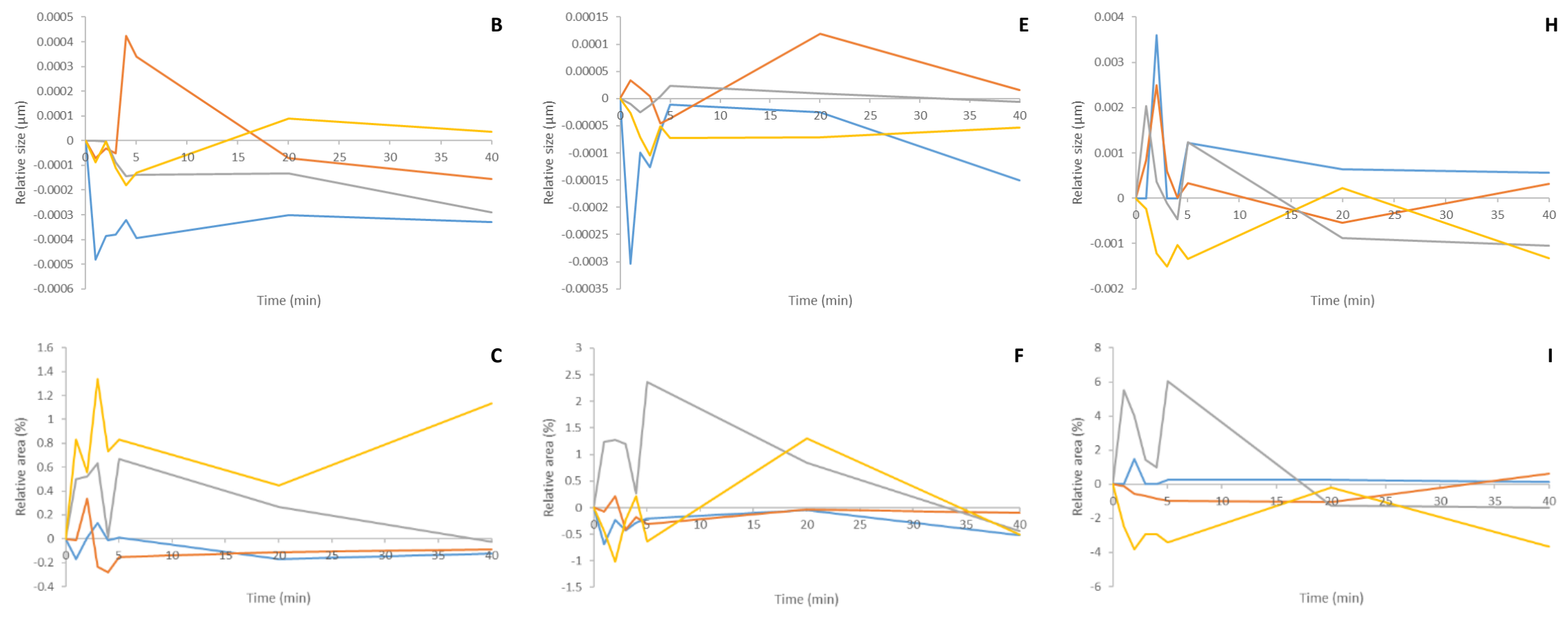

F

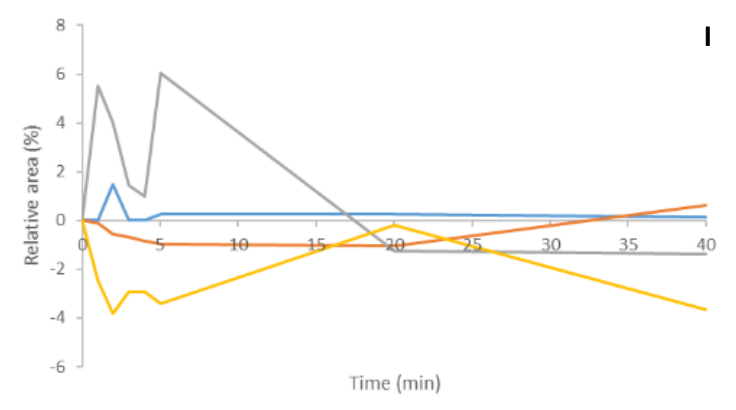

Figure S4. Effect of a high dose $(500 \mu \mathrm{g})$ of fengycin on liquid disordered $\left(\mathrm{L}_{\mathrm{d}}\right)$ domain characteristics and distribution of lipid bilayers. Bilayers contained 3:1 DOPC:DPPC with 0 (blue), 3 (orange), 6 (grey), and 12\% (yellow) ergosterol. Fengycin added at the 0 minute marks. A-C. Small domains $(<1 \mu \mathrm{m})$; D-F. Medium domains $(1-10 \mu \mathrm{m})$; G-I. Large domains $(>10 \mu \mathrm{m})$. A, D, G. Relative domain count; B, D, H. Relative domain size; C, F, I. Relative domain area. 